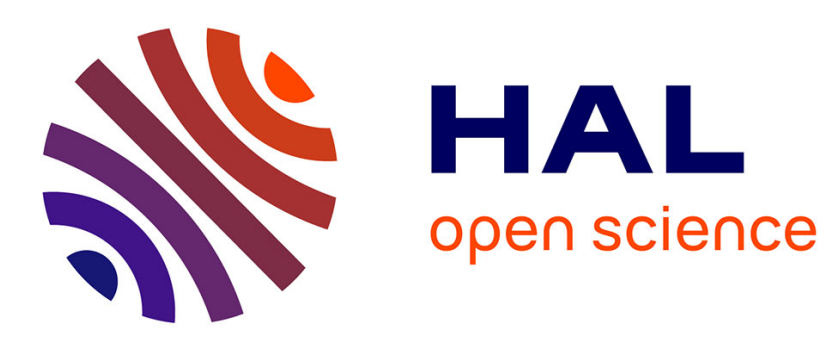

\title{
The filling law: A general framework for leaf folding and its consequences on leaf shape diversity
}

\author{
Etienne Couturier, Sylvain Courrech Du Pont, Stéphane Douady
}

\section{To cite this version:}

Etienne Couturier, Sylvain Courrech Du Pont, Stéphane Douady. The filling law: A general framework for leaf folding and its consequences on leaf shape diversity. Journal of Theoretical Biology, 2011, 289, pp.47. 10.1016/j.jtbi.2011.08.020 . hal-00739264

\section{HAL Id: hal-00739264 \\ https://hal.science/hal-00739264}

Submitted on 7 Oct 2012

HAL is a multi-disciplinary open access archive for the deposit and dissemination of scientific research documents, whether they are published or not. The documents may come from teaching and research institutions in France or abroad, or from public or private research centers.
L'archive ouverte pluridisciplinaire HAL, est destinée au dépôt et à la diffusion de documents scientifiques de niveau recherche, publiés ou non, émanant des établissements d'enseignement et de recherche français ou étrangers, des laboratoires publics ou privés. 


\section{Author's Accepted Manuscript}

The filling law: A general framework for leaf folding and its consequences on leaf shape diversity

Etienne Couturier, Sylvain Courrech du Pont, Stéphane Douady

PII: S0022-5193(11)00423-1

DOI: doi:10.1016/j.jtbi.2011.08.020

Reference: YJTBI 6592

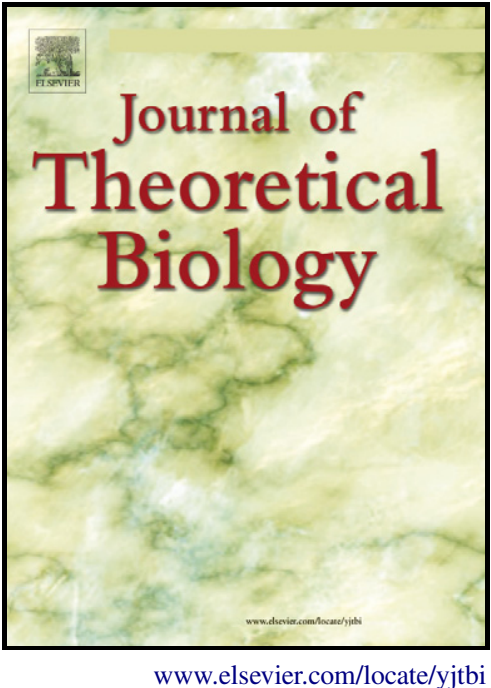

To appear in: Journal of Theoretical Biology

Received date: $\quad 8$ October 2010

Revised date: $\quad 17$ August 2011

Accepted date: 18 August 2011

Cite this article as: Etienne Couturier, Sylvain Courrech du Pont and Stéphane Douady, The filling law: A general framework for leaf folding and its consequences on leaf shape diversity, Journal of Theoretical Biology, doi:10.1016/j.jtbi.2011.08.020

This is a PDF file of an unedited manuscript that has been accepted for publication. As a service to our customers we are providing this early version of the manuscript. The manuscript will undergo copyediting, typesetting, and review of the resulting galley proof before it is published in its final citable form. Please note that during the production process errors may be discovered which could affect the content, and all legal disclaimers that apply to the journal pertain. 


\section{The filling law: a general framework for leaf folding and its consequences on leaf shape diversity}

Etienne Couturier, Sylvain Courrech du Pont, Stéphane Douady

Laboratoire Matières et systèmes complexes (MSC)

UMR 7057 CNRS/Université Paris-Diderot

10 rue Alice Domon \& Léonie Duquet

75205 Paris Cedex 13, France

\section{$\underline{\text { Abstract }}$}

Leaves are packed in a bud in different ways, being flat, rolled, or folded, but always filling the whole bud volume. This « filling law » has many consequences, in particular on the shapes of growing folded leaves. This is shown here for different types of folding and packing. The folded volume is roughly a part of an ellipsoid, with the veins on the outside rounded face and the lamina margin on the adaxial plane. The veins on the abaxial side protect the fragile lamina inside. The first general consequence of the folds and the space limitation of the lamina growth is the presence of symmetries on the leaf shape, and the second is the quantitative relationships between the sizes of the lobes and sinuses. For particular geometries, the leaf lamina can be limited by lateral veins, creating spoon-like lobes, or tangent cuts, creating asymmetrical wavy perimeters. Changes in the packing between different cultivars correspond to changes in the mature leaf shapes. Each particular case shows how pervasive the geometrical consequences of the filling law are.

\section{1 - Introduction}

Leaves present many different shapes. They can be grouped in various categories, for instance simple, with lobes (palmate), with leaflets (compound), or dissected with holes. On one single plant, the leaf shapes can vary strongly, which is called heterophylly. Despite this diversity, some common features can be intuitively recognized even among the leaves from distant phylogenic origins. In other words, there are more variations within few well delimited types than a real liberty of shapes. For instance, folded palmated leaves are all similar, which comes from an apparent common relationship of the respective lobes' dimensions.

Leaves first appear as primordia, little bulges around the centre of the apical meristem [Hofmeister (1868)]. Very quickly a primordium expands ortho-radially, as a surface wrapping around the stem axis. From the beginning, they present a fundamental asymmetry: the side toward the stem axis (i.e. the adaxial side) will become the smooth and shiny upper side of the leaf turned toward the light; the other side, the side of the primordia toward the outside (i.e. the abaxial side), presents protruding hairs and veins and will become the lower side of the mature leaf [Esau (1977)]. 
The lobes' appearance and development can be considered as reiteration of primordia development. Lobes are secondary primordia protruding from the margin of the primordia surface, as reiteration of the leaf primordia itself [Bell (1991)]. At the molecular level, it has been shown that for lobed leaves, the same genes are expressed in both cases: the gene CUC is expressed at the boundary between the primordia and the meristem and at the separation between the lobes and the primordia [Blein (2009)].

As the development of a lobe is necessarily linked with the development of a vein, lobes and veins have the same hierarchy. Each main lobe corresponds to a major vein ending at its tip. Similarly, secondary veins branch out from them, possibly developing secondary lobes, and so on and so forth.

While the mechanisms underlying initiation of lobes and veins begin to be understood by developmental biologists, what regulates their final size and shape remains largely unclear. We have shown in a previous article that for some palmated leaves, a key feature of this regulation is that these leaves develop folded inside the bud [Couturier (2009)].

All the buds from different plants, taken in the general meaning of the compact organisation of leaves and stipules around the meristem apex (and not only immature leaves protected by scales [Bell (1991)]), are very well organized. The internal bud space is perfectly filled with the successive primordia, which all occupy a volume with the same shape, but in decreasing sizes. The shape of the volume occupied by a leaf does not depend much on species: it is roughly a quarter of an ellipsoid. The curved face and the face of the largest plane are on the abaxial side and the adaxial side, respectively, and the smallest face is essentially the base of the leaf.

The compaction of the leaf inside the bud was noticed early on [Adanson (1763)] and used as a criterion of classification ("19e syftême. feuilles; leur enroulement \& dévelopement"). The importance of the packing was best emphasized by Lamarck [Article IX, §82, p. 106, Lamarck \& DeCandolle (1815)]: "Les feuilles existent dans le bourgeon, munies de toutes leur nervures, mais non développées ; elle y sont placées de manière à y occuper le moins d'espace possible : cette disposition varie dans différens (sic) végétaux, car elle est déterminée par la position respective des feuilles et la disposition de leur nervure." ("Leaves exist in the bud, with all their veins, but undeveloped; they are placed in order to occupy the least possible space: this arrangement varies in different plants, as it comes from the respective positions of leaves and the arrangement of their venation.”). The different ways of packing were also mentioned later on, as in [Jussieu (1839)]. Following Adanson, this particularity was used more for classification, and sorting of plants with their respective prefoliation was done by [Clos (1870)], or more recently by [Cullen (1978)] (who was unaware of Clos' work). This phenomenon was further summarized in [Bell (1991)]. But none of these works developed Lamarck's remark that leaves are packed to best occupy the space.

Hofmeister was the first to really consider the dynamics of development in plants. After 
focusing on the successive appearance of primordia and their geometry (i.e. phyllotaxis), he wrote two book chapters on leaf expansion and the leaf arrangements within the bud [§13-14, Hofmeister (1868)]. Considering how a primordium extends, essentially laterally, he highlighted the fact that leaves have a given space to develop (flat or folded in two), essentially delimited by the stipules on the sides. However, this developmental work remained inspired by the idea that the folding or rolling of leaves and their shapes are fixed a priori.

More recently, Williams devoted a whole book to quantification of leaf development [Williams (1975)]. Although he noticed "the extraordinary tight and very beautiful packing of the successive members of the apex" (of Ficus elastica, p. 149), he cautiously introduces the idea of physical constraints, and that pressure from the other organs can "mold" the development in some cases. In general, he limited this effect to the determination of the relative growth rates of the organs $(\S 8)$, and insisted on the simple solution of applying exponential growth to all organs in the bud (i.e the growth rate of each organ is roughly proportional to its distance to the central meristem), in order to keep a tightly filled bud during the whole growth process.

Except from this work, the development of the leaf, in particular when it is enclosed within the bud, was roughly forgotten, and scientists have since focused on the primordium appearance at the stem apical meristem, from [Snow \& Snow (1962)] to [Hamant et al. (2008)], and in the late phase of leaf development free from the bud, or in the species in which the leaves grow entirely free from the bud, such as tobacco, cotton and Arabidopsis Thaliana. In the case of cotton [Dolan et al. (1998)], the leaf folding and its connection with lobe formation were never mentioned.

The only case where the importance of folds has been recognized and studied in detail was in leaves of palm, a monocotyledon [Kaplan et al., Dengler et al.(1982)]. But the connection of the folds and leaf shape to the available space in the bud was not made. For dicotyledons, there is, to our knowledge, only one study on leaf folds with Japanese maple [Kobayashi et al. (2000)]. The vague similarity of lamina folds in young beech or hornbeam leaves with a particular type of paper folding (Miura's origami) was also underlined [Kobayashi et al. (1998), Mahadevan \& Rica (2005)], even though they recognized its superficiality because, contrary to the paper origami, the leaf central vein is never bent (see [Kobayashi et al. (1998)] Figure 5).

As stated by Lamarck, if all the leaves occupy the volume in the bud similarly, they have different ways of filling it. What is surprising, however, is that the leaf shape corresponds exactly to how the primordia filled the bud when folded (or rolled). As a consequence, and to our knowledge it was not highlighted before, this general compaction requirement directly links the leaf shape to the volume the leaf occupied and the way it was packed inside the bud. We call this the "shape-filling law". We show that this law is generally applicable to many species and presents its geometrical consequences on leaf shapes, in particular on folded leaves. Before showing many particular cases, for 
the sake of a complete article, we will recall, although in a different way (\$2.2 and 2.4-1), our first results with maples as examples [Couturier et al. (2009)].

On the mathematical part, Demaine [Demaine (1998)] has shown that any polygonal shape can be obtained theoretically by folding of a plane and with only one straight cut through it. However, this work remains purely two dimensional and does not take into account the real thickness of the delimited (cut) object, or the spatial delimitation of the delimited surface. On the contrary, it is important to realize that premature leaves are not zero-thickness surfaces with a thick lamina and veins and actually occupy a volume. Moreover, Demaine's work provides a way to find the folds that would give rise to the desired cut perimeter, whereas the problem of the leaf shape is the inverse. The folds precede and are independently determined by the leaf growth (folding around the veins and lamina growth). In this article, we will thus show that the actual known folding of the leaves indeed correspond to the final perimeter observed, with some particular three dimensional effect, and in particular folds of non-zero Gaussian curvature surfaces.

\section{$\underline{2 \text { - Results }}$}

\section{1 - Compactness of the bud}

Many dicotyledonous leaves of various phylogenetic origins are folded during their development. Even if these plants have different phyllotactic patterns, their buds have similar organisations. They fill a part of roughly an ellipsoid volume, with the veins on the outside, rounded part of the ellipsoid, and the lamina, always as locally flat as possible, folded inside. The leaf margins lay on a flat border, either on an axial limitation plane or inside its own volume. The differences in the organisation just come from the different objects in the bud delimiting the internal border. In the Acer platanus type, the object is the margin of the opposite folded leaf (Figure 1a). In the Morus platanifolium type, the objects are the stipules of the next leaf, which are joined in a closed envelope that protects the next younger leaf (Figure 1b). In the Pelargonium cuculatum type, it corresponds to the bottom part of an older leaf (Figure 1c).

Figure 1: Similar structures in different kinds of buds. The external part is always constituted by veins and the lamina is folded inside. a. An Acer pseudoplatanus bud. The folded leaf margin lays on a plane against the opposite leaf margin. b. A Morus platanifolium bud. The folded leaf margin lays on a plane against the stipules, which envelopes a smaller bud. c. A Pelargonium cuculatum bud. The folded leaf margin lays on a plane against the lower part of an older leaf. d. Gunera manicata. Side view of the leaf. d'. Front view of the same leaf. The leaf constitutes its own bud, and the lamina is delimited by the central axis (as seen in Figure $2 \mathrm{c}$ ).

In the fourth type of bud organisation typical of tropical climate, the leaf is globally wrapped around itself, so that inside delimitation is not applied by another leaf but by the leaf itself. In this way, each leaf behaves as an autonomous bud (Figure 1d-d'), where the leaf margin is limited on a central axis. This is essentially the case for compound leaves, with each folded leaflet having a margin on a central internal leaf plane. These four types of organisations enable perfect filling and tilling of the 
internal bud space (Figure 2a-b-c).

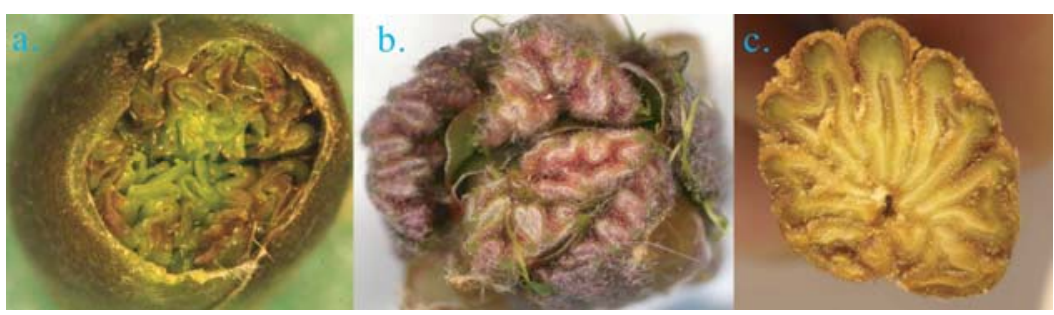

Figure 2: Three cuts of the three different bud kinds : a perfect and rational filling of the bud. a. Opposite phyllotaxy, Acer pseudoplatanus. b. Spiral phyllotaxy, Quercus Rubra. c. Autoclosing leaf, Tetrapanax papyrifer.

\section{2 - Basic perimeter symmetry}

In all these types, the minimum consequence of this organisation is that even if the actual folding occurs in three dimensions with thick veins, the margins of two consecutive main lobes are folded on the same line. On both sides of a fold, the leaf margins lay on the same line, thus each fold corresponds to the symmetry axis of the perimeter in the unfolded leaf, either for a sinus or a lobe. The abaxial folds (away from the stem axis), which correspond to lobes, form along main veins (Figure 3a). The adaxial folds (toward the stem axis), which correspond to sinuses, form in the lamina (Figure $3 b$ ). The lamina expands in the zone between two main veins, where the smaller secondary veins join together (Figure 3c). We call these lamina folds "antifolds" or "anti-veins".
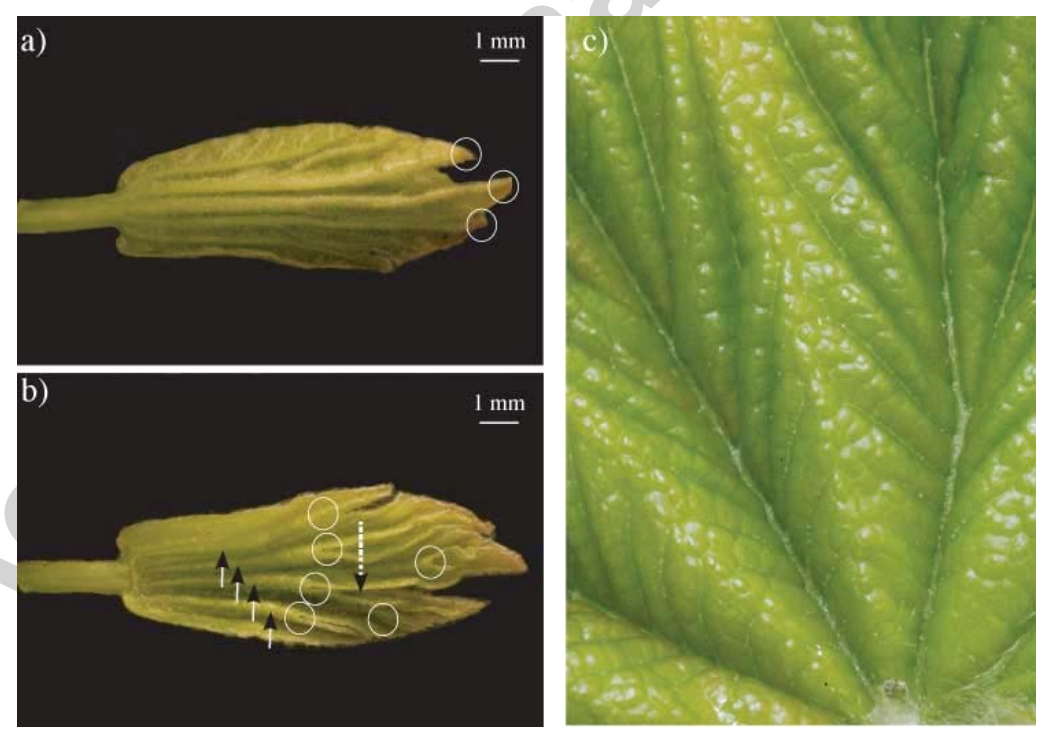

Figure 3: Folded immature leaf extracted from a bud of Acer campestre. a. The abaxial side shows the folds running along veins and ending at peaks (circles). b. The adaxial side shows the lamina folds (arrows) running along (immaterial) anti-veins and ending at sinuses (circles). Peaks and sinuses lay in the contact plane of the pair of leaves (Figure 1), but while peaks are at the extreme of this contact surface, sinuses are positioned inside. c. Upward rising antifolds of Acer pseudoplatanus between the main veins. Only the last order veins, emanating from surrounding main veins, join along the antifold.

As the symmetry of the leaf margin around a fold or antifold is the geometrical constrain for packing, it works for all folded species independently of their phylogeny (Figure 4). 


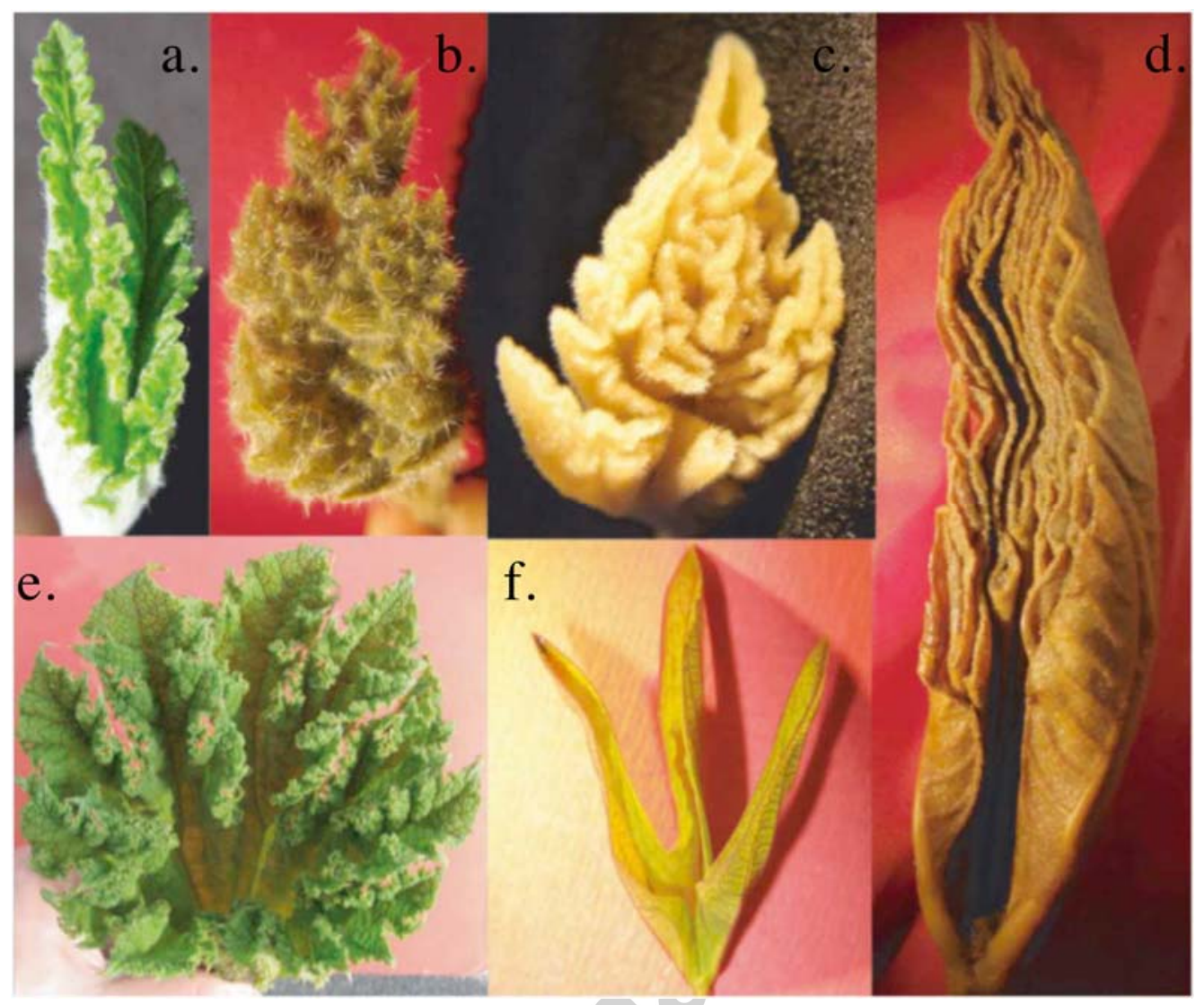

Figure 4: Front view of different folded-and-cut leaves . a. Ribes Nigrum. b. Pelargonium cuculatum. c. Malva Sylvestris. d. Tetrapanax papyrifer. e. Gunnera manicata. $f$. Passiflora. They are of different phyllogenetical origins: a belongs to Saxifragales, b to Geraniales, c to Sapindales, d to Apiales, e to Gunnerales, and $\mathrm{f}$ to Malpighiales.

This symmetric property, built during the leaf development in the bud, is preserved in the expansion of the mature leaf. The veins, which correspond to the abaxial folds, are the medial axes of the lobe even for mature leaves (Figure 5a,c). The antifolds correspond also to the symmetry of the medial axes of the sinuses (Figure 5b). Because of the preservation of these symmetries during the leaf expansion, the leaves of these species can be refolded from the unfolded mature states (Figure 6). The delimitation of the folded lamina by an external plane is similar to the case of a sheet of paper folded and cut. We thus call this limitation Kirigami ("folded-cut-paper" in Japanese, see Figure 7). 


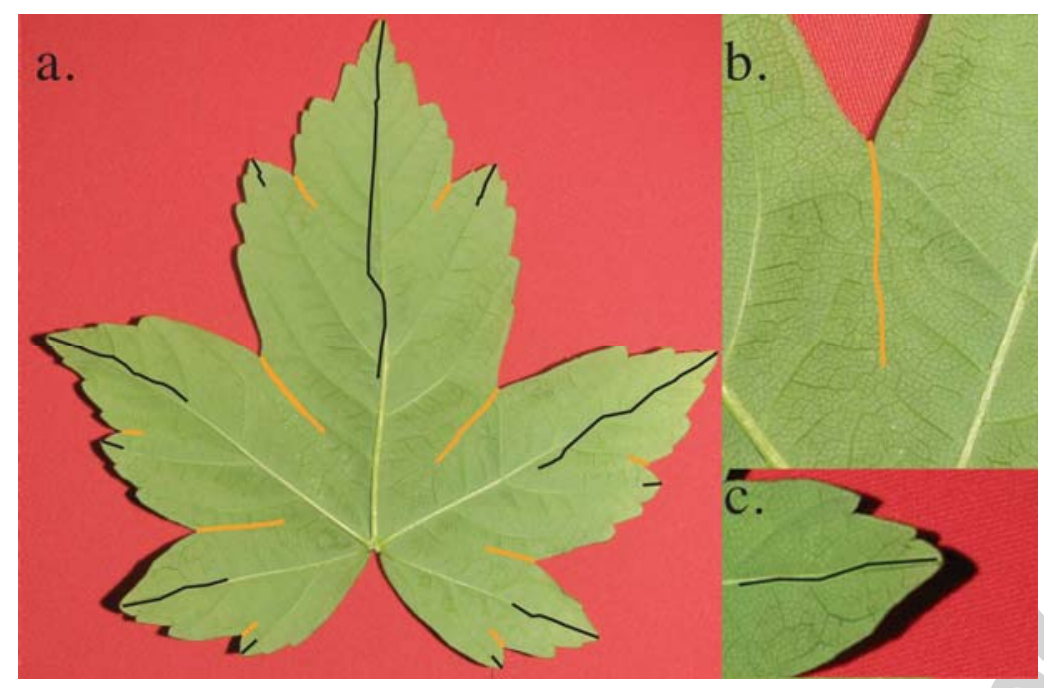

Figure 5: a. An Acer pseudoplatanus leaf with symmetry lines drawn. Black lines marks the medial axes of the lobes, derived from the perimeter. Orange lines are the symmetries around the sinus minmum of the medial axis of the sinus. b. The orange line corresponds to a lamina, or an antivein, fold. $\mathrm{c}$. The black line corresponds to a vein fold. The black lines are not exactly on the veins, because they were determined from the leaf boundaries, and thus were influenced by the secondary perimeter variations.
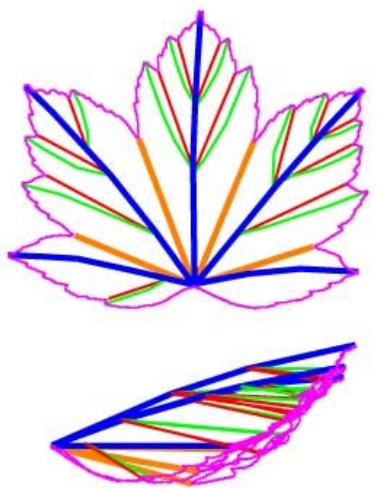

c)

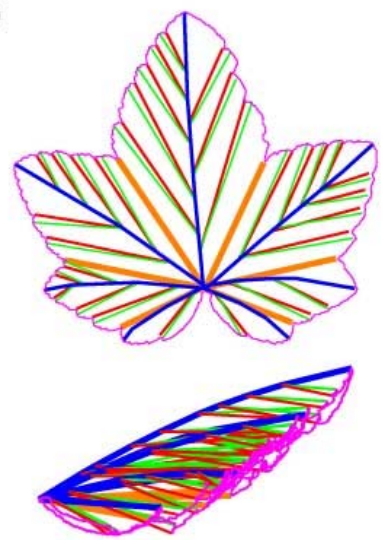

b)
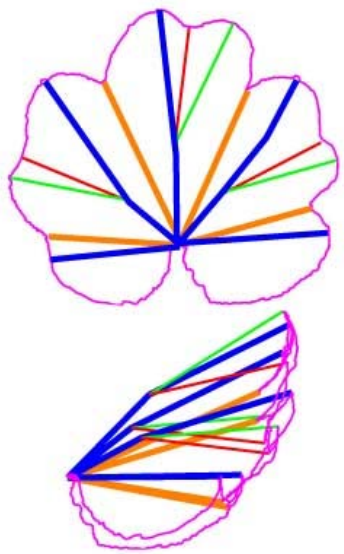

d)

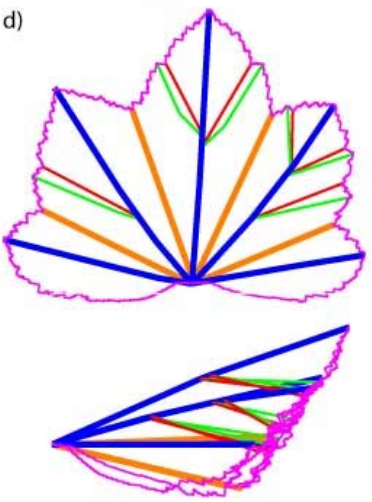


Figure 6: Numerical folding of several species. a. Acer pseudoplatanus. b. Gunera manicata. c. Ribes Nigrum. d. Malva sylvatica. Note that the perimeter refolds on a single line even for asymmetric leaves, as in $\mathrm{d}$. See Annex for the method of numerical folding.

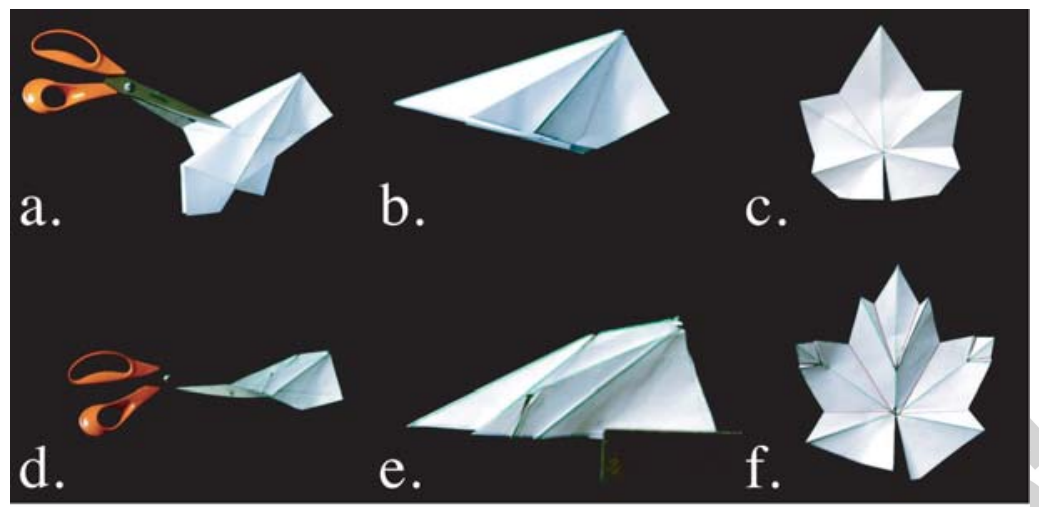

Figure 7: Examples of cut, folded paper in the shape of a leaf (Kirigami -'(folded)-cut paper' in japanese) a. A rectangular sheet of paper with five folds originating from the same point (petiole) is then cut in a straight line with scissors. The folded margin is complicated. b. Same folded sheet of paper, cut. The folded margin lays on a simple surface. c. Same sheet, unfolded. Folds correspond to sinuses and lobes. d. Same sheet folded with secondary folds. e. Same sheet, cut f. Same sheet, unfolded. Secondary folds correspond to secondary lobes.

\section{3 - Lamina Antifold symmetry}

Leaves have other symmetries originating from their growth, during which they are folded in a particular way. For all the folded leaves, the main veins constitute an outside envelope of contiguous veins around the lamina, protecting it like armour (Figure 8 a-d). In order for a vein to become contiguous to its neighbouring one, the flat lamina has to be folded symmetrically (Figure 8e). The lamina antifold is thus the bisector of the two surrounding vein folds. For the same reason, in case of more than two vein folds around a lamina, the lamina antifolds are constituted by the bisectors of successive pairs of vein folds (Figure 8f), even if in this case the lamina, locally flat between the lamina folds, is no more a globally flat surface. In the common cases of antifold formation between two secondary veins, it takes the typical shape of a wedged roof (see Figure 8f'), which in particular has a non-zero Gaussian curvature. This is the reason why these leaf shapes, during their development, cannot be obtained simply by folding paper. As the antifolds correspond to the bisectors of the veins, the sinuses, which are at the end of the antifolds, are on the bisectors of the veins (Figure 8e-f). The final contour of the leaf is strongly constrained by this property. 


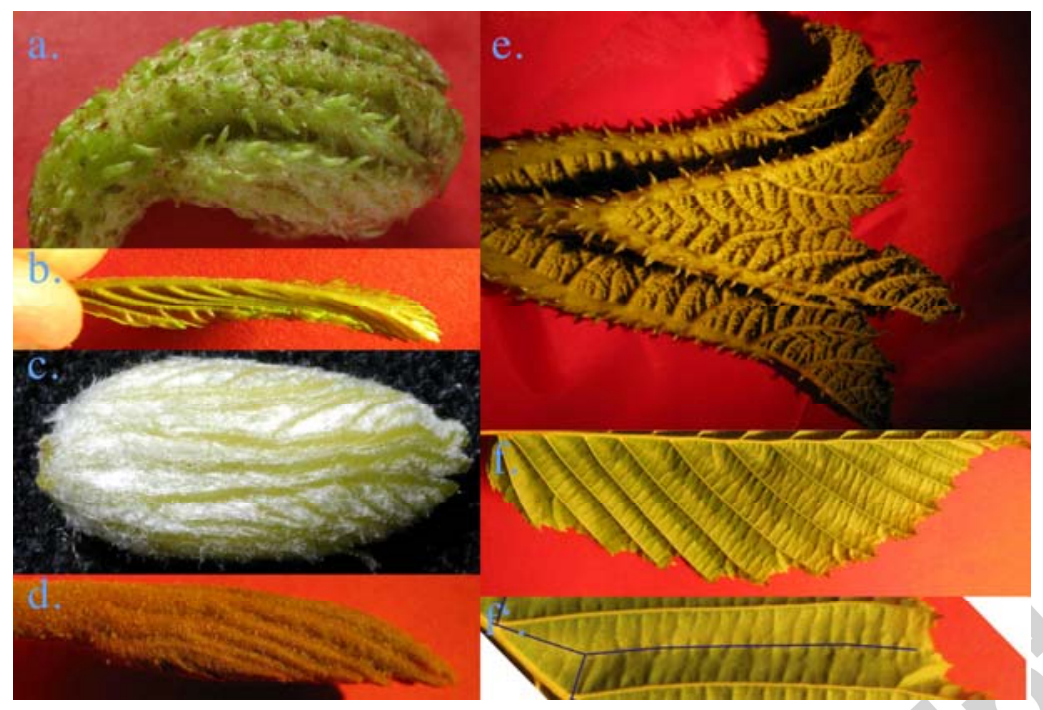

Figure 8: Veins are on the outside of the folded leaf, protecting the lamina with antivein folds inside. a. Gunnera manicata. b. Carpinus betulus. c. Acer pseudoplatanus. d. Tetrapanax papyrifer. e. As the antifold folds a vein on its neighbouring vein, and the lamina is locally flat, the antifold is situated at the bisector of two adjacent veins. f. f'. For the same reason, the antivein fold which is situated between three veins, is constituted by three pieces of bisectors.

al.

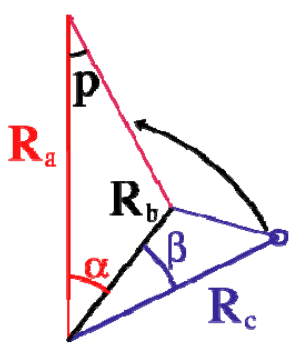

bl.

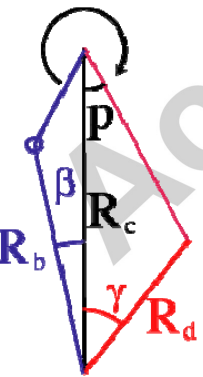

a2.

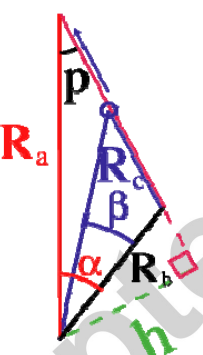

b2.

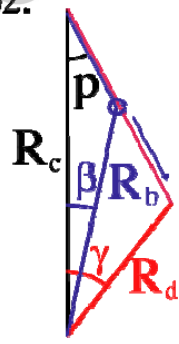

a3.

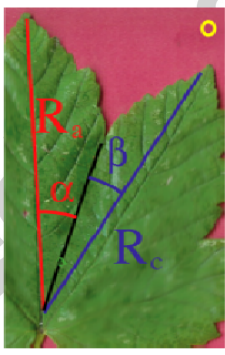

b3.

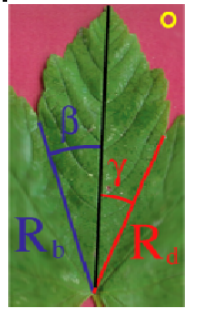

a4.

b4.

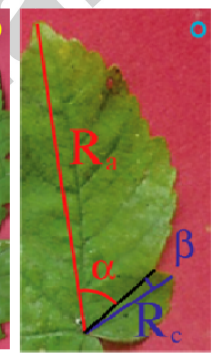

a5.
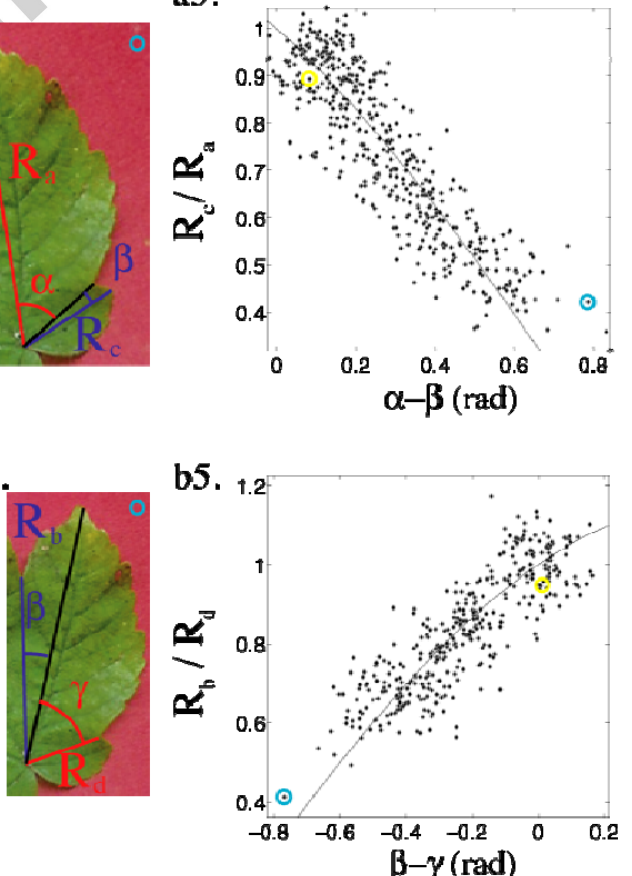

Figure 9: Geometric relationships between two successive lobes and sinuses due to the Kirigami property. a1:

Two consecutive primary lobes have veins of lengths $R_{a}$ and $R_{c}$. They respectively make angles $\alpha$ and $\beta$ with the anti-vein between them, of length $R_{b}$. b1: The vein of length $R_{c}$ is surrounded by two anti-veins of lengths $R_{b}$ and $\mathrm{R}_{\mathrm{d}}$. These are respectively making angles $\beta$ and $\gamma$ with the vein. a2: From the different expressions of the height $\mathrm{h}$, one can derive quantitative relationships between these elements (see text). a3-4 : two extreme examples from real leaves. a3: When lobes have equal length the anti-vein is then the bisector $(\alpha \sim \beta)$. a4: When the smaller 
lobe is close to the anti-vein $(\beta<<\alpha, a 4)$, its length is that of the antivein. In general, the antivein is always closer to the smaller lobe. a5: Quantitative relationship between two successive lobes and sinuses due tothe Kirigami property, for 121 Acer pseudoplatanus (sycomore) leaves. Length ratio $\left(\mathrm{R}_{\mathrm{a}} / \mathrm{R}_{\mathrm{c}}\right)$ of two consecutive main veins in function of the difference $(\alpha-\beta)$ between the angles they make with the anti-vein. $b$ : same figure for two antiveins on the side of the central vein lobe. b5 : Length ratio $\left(\mathrm{R}_{\mathrm{b}} / \mathrm{R}_{\mathrm{d}}\right)$ of two consecutive main anti-veins in function of the difference $(\beta-\gamma)$ between the angles they make with the vein. When anti-veins are equal in length the vein is then the bisector $(\beta=\gamma, \mathrm{b} 3)$. The vein always runs closer to the smaller anti-vein (b4).

\section{4 - Quantitative perimeter symmetry}

\subsection{1 - Primary folds}

Different kinds of folding will have different consequences on the leaf shape. The simplest leaf folding, called « radial folding », which has folds radiating from the same point (the end of the petiole), corresponds to the simplest way of folding a sheet of paper. To represent the limitation of the lamina from the enclosing volume (i.e. the filling law), these folds are cut along a plane. This adaxial plane determines the folded margin of the leaf. After unfolding, the geometry is very simple (Figure $7 \mathrm{c})$.

To find the formula, we superimposed the triangle delimitated by $R_{a}$ and $R_{c}$ and the perpendicular $\mathrm{h}$ to the leaf lamina (Figure 9 a2). One can simply express the value of this height, which depends on the three lengths and angles. The first relation is $h=\sin (p) R_{a}$. Given that the sum of the angle of a triangle is $\pi$, one can also write $h=\sin (p+\alpha) R_{b}$, and $h=\sin (p+\alpha-\beta) R_{c}$. Setting the first and last expressions equal yields the equation:

$$
R_{a} / R_{c}=\sin (p+\alpha-\beta) / \sin (p) \text {. }
$$

In figure 9, we used the average of all the $\mathrm{p}(\mathrm{s})$ from all the groups of two consecutive lobes to compare the formula with the measurements.

Because the leaf margin lays on the same line on the both sides of a fold, the longest is the one whose vein makes the biggest angle with the separating anti-vein fold for two consecutive lobes (Figure 9a). Similarly, for two sinuses around a middle vein, the smaller of two adjacent sinuses makes the bigger angle with the middle vein (Figure 9b). This qualitative rule explains why all « radially folded » leaves, called the palmate leaves, have a similar set of traits in their shapes, regardless of their phylogenetic origins (Figure $10 \mathrm{a}-\mathrm{c}$ ). 


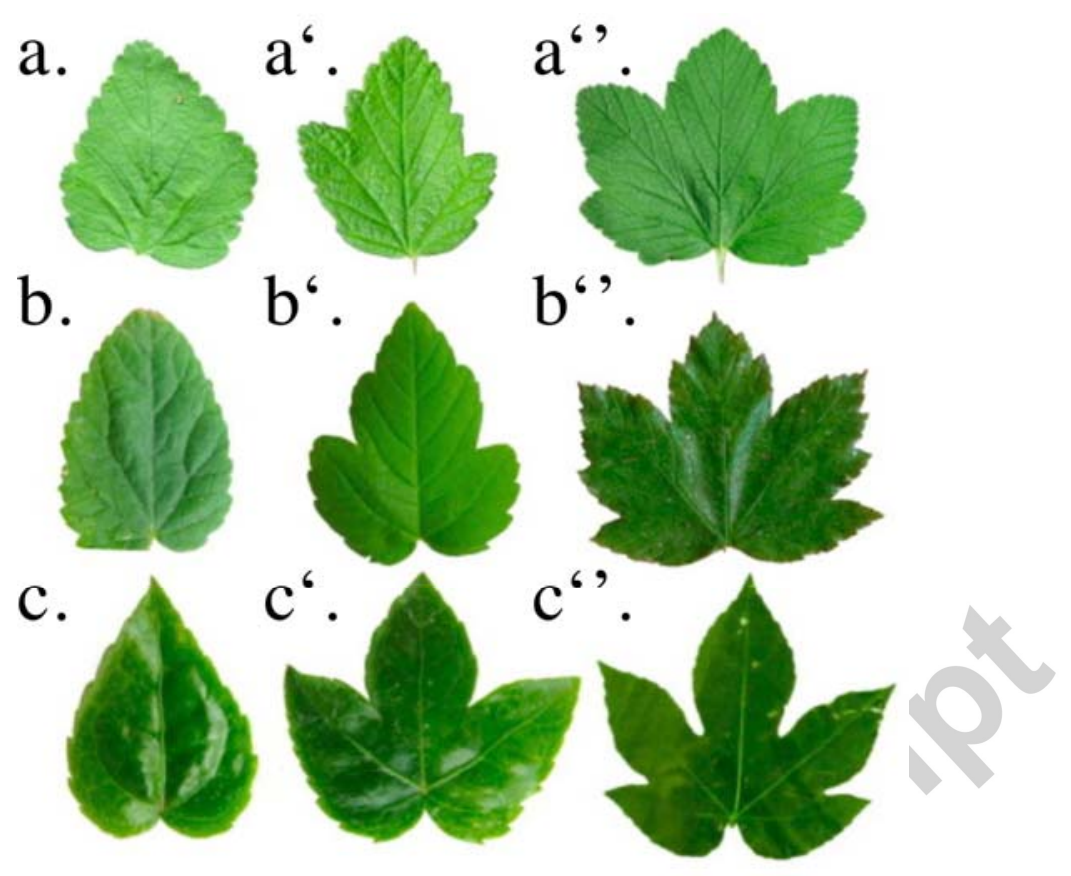

Figure 10: Fold-and-cut leaves from different phylogenetic origins have the similar traits of leaf shapes. a-a": Ribes Nigrum leaves, Saxifragales. b-b": Acer pseudoplatanus leaves, Sapindales. c-c": Fatsia japonica leaves, Apiales.

\subsection{2- Secondary folds}

The second step of complexity in folding is the creation of secondary folding along a previous fold. After cutting, the secondary folds will create secondary lobes at the margin of the unfolded sheet of paper (Figure 7f). The different dimensions of these secondary lobes will be linked quantitatively. For instance, using Figure 11a, we can predict the opening angle $\psi_{2}$ using the precedent opening angle $\psi_{1}$, and the angles $\alpha_{1}, \beta_{2}, \gamma_{2}$ between the veins and antiveins. For this purpose, we need to find the value of the angle at the stage where the leaf was folded (Figure 11b). As in the case of numerical folding, we have used the angles $\beta_{2}^{\prime}, \gamma_{2}^{\prime}$, which are the best approximations from $\beta_{2}$ and $\gamma_{2}$, but which (unlike $\beta_{2}$ and $\left.\gamma_{2}\right)$ follow the relation of Kobayashi and thus are foldable in a plane: $\beta_{2}^{\prime}=\left(\pi+\beta_{2}-\alpha\right.$ 2) $/ 2, \gamma_{2}^{\prime}=\left(2 \gamma_{2}+\beta_{2}-\alpha_{2}-2 \pi\right) / 2$ (see Annex).

We consider the angles of ADCB of the figure $11 \mathrm{~b}$ :

$\alpha_{1}+\left(\beta_{2}^{\prime}-\gamma_{2}^{\prime}\right)+\left(\pi-\psi_{1} / 2\right)+\left(\pi-\left(\pi-\psi_{2} / 2\right)\right)=2 \pi$.

We can rewrite it:

$$
\alpha_{1}+\beta_{2}^{\prime}-\gamma_{2}^{\prime}-\psi_{1} / 2-\pi+\psi_{2} / 2=0 .
$$

Thus:

$$
\psi_{2}=2 \pi+\psi_{1}-2 \alpha_{1}-2 \beta_{2}^{\prime}+2 \gamma_{2}^{\prime} \text {. }
$$

To test this prediction, we measured the angles shown on Figure 11a on five leaves of Tetrapanax papyrifer. We calculated the refolding angle by using the nearest angle, obeying the theorem of Kobayashi (see Annex). Using these calculations, we could predict the angle, though the 
measurements nearly always exceeded the predicted value (Figure 11c). This is probably because in our model we only considered the curvature of the central main vein that resulted from the secondary folds. As shown in Fig 8d, the central main vein is also curved between the folds. Thus, our model underestimated the curvature of the central vein and overestimated $\alpha$ and $\beta$ (imagine the first red segment of the main vein curved outside in Figure 11a), and the formula underestimated $\psi$.

a.

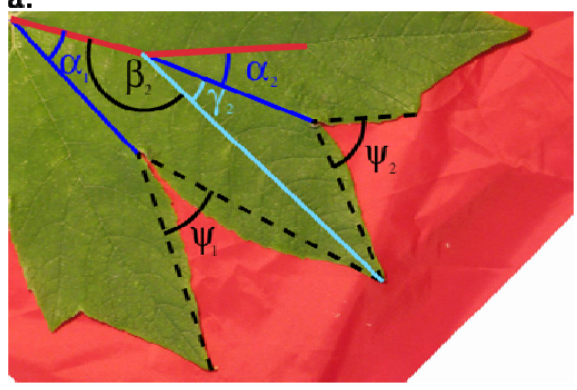

b.

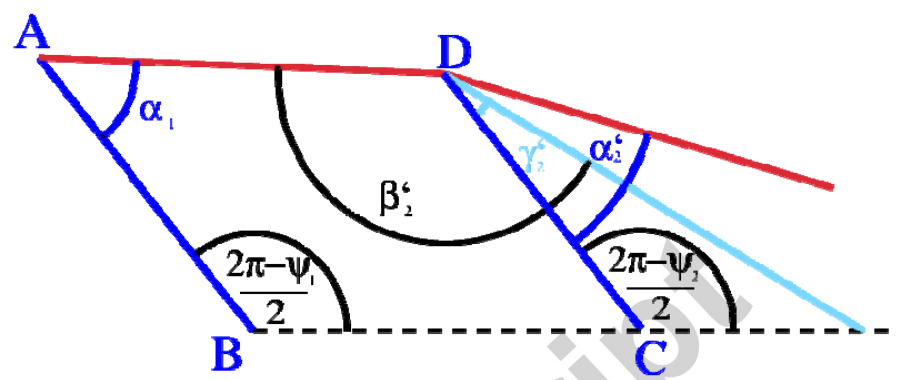

c.

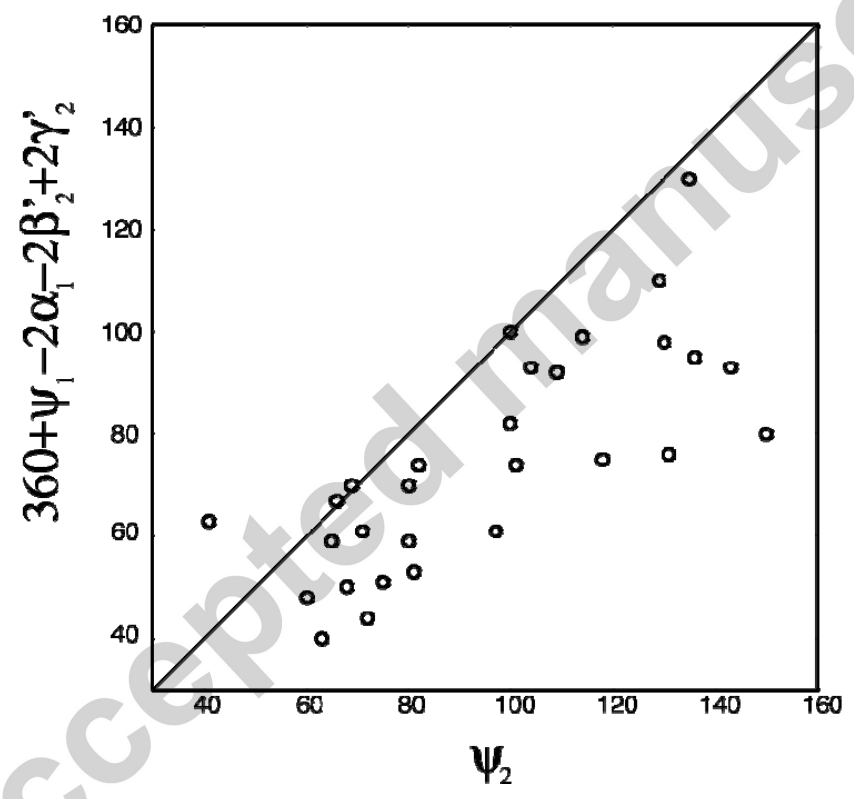

Figure 11. Quantitative relationship between successive secondary folds. a: A lobe of a Tetrapanax papyrifer leaf. The red line corresponds to the main vein. The cyan line corresponds to the secondary vein. The blue line corresponds to the antifold. Notation for the measured angle. $\alpha_{1}, \varphi_{1}, \alpha_{2}, \beta_{2}, \gamma_{2}$ and $\varphi_{2}$. $b$ : The same lobe, folded. $\beta_{2}^{\prime}$ and $\gamma_{2}^{\prime}$ are the nearest angle from $\beta_{2}, \gamma_{2}$ that you can fold in a plane, which is why we used them to make the prediction. c: Data obtained by measurement on five leaves of Tetrapanax papyrifer, with $\psi_{2}$ on the abscissa and the prediction made by assuming that all the lobe refolds along the same line on the ordinate.

These quantitative constraints have a simple consequence on leaf shape: the opening angle of each sinus $(\psi)$ successively increases along the lobe (Figure 12b-c). If we take into account the folded phase of development, the reason for this observation becomes clear. The key factor is that the secondary folds bend the central vein (Figure 12a). Measurements showed that the bending is regular: $\beta=166^{\circ} \pm 5^{\circ}$ (30 folds). Measurements also showed that angle $\alpha$ between the vein and the 
antifold do not depend on their positions along the main fold, indicating that development of the antifold is uniform: $28.1^{\circ} \pm 5^{\circ}$ for the first angle along the lobe ( 22 folds), $26^{\circ} \pm 6^{\circ}$ for the second one ( 22 folds) and $27.4^{\circ} \pm 5^{\circ}$ for the third ( 8 folds). Then, because of the curvature of the main vein that results from these secondary folds (Figure 12a), the angle), between the antifold and the cut plane, decreases along the lobe (Figure 11c). As the angle) is smaller along the lobe, the angle of sinus opening $\Pi$, which is equal to $2 \pi-2$ ), becomes larger along the successive lobes (Figure 12c).

Because of this central vein curvature, all the secondary lobes make a different angle with the adaxial plane. Rather than having a uniform shape, as would result from a simple reiteration process, they adapt their shape to this local geometrical environment as the filling law predicts.

a.

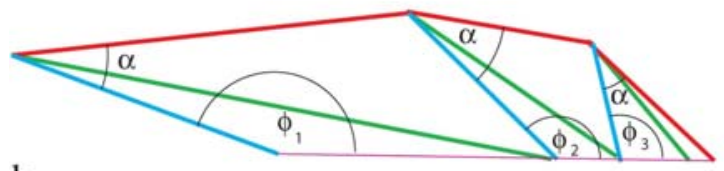

b.
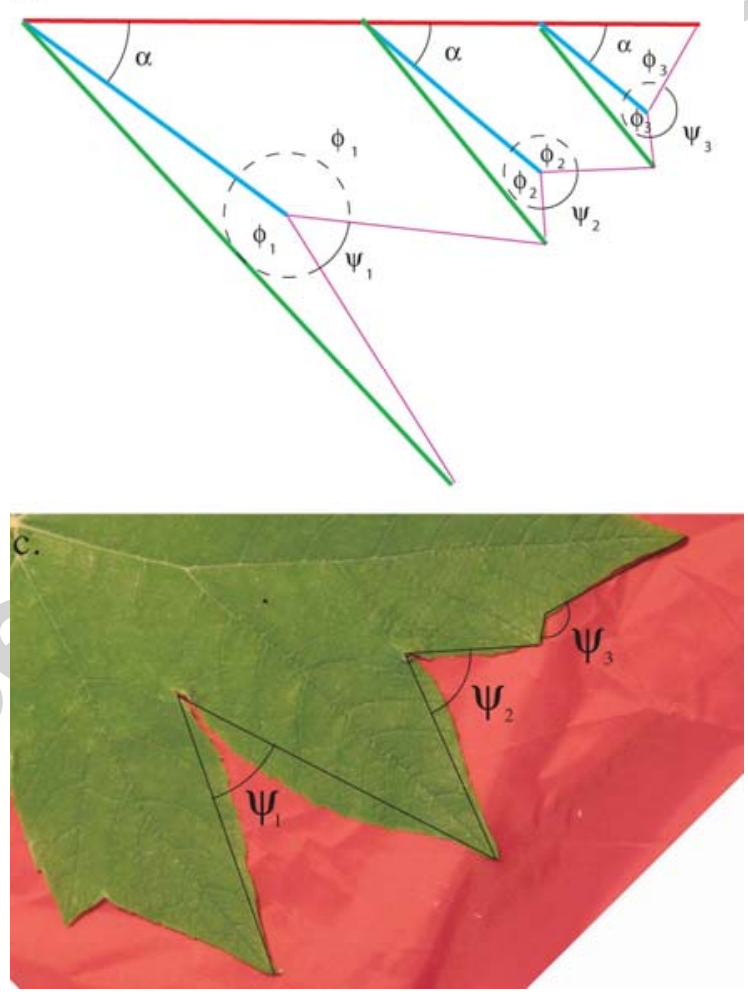

Figure 12 : Consequence of secondary folds on the opening angles of the successive sinuses. a: Main vein (red) is bent by each secondary fold (green) and antifold (cyan). The angle between antifold and vein does not depend on its positions along the vein. For this reason, the angle $\phi$ between the antifold and the cut decreases along the vein. b: For this reason, the opening angle $\psi$ of the sinus increases along the lobe. c: Three successive sinuses along a lobe of Tetrapanax papyrifer. The opening angle increases toward the apical tip: $\psi_{1}<\psi_{2}<\psi_{3}$.

\section{5 - Non planar folding \& Adaxial limitation}

All the precedent shapes of leaves can be imitated with a flat sheet of paper (which has null Gaussian 
curvature) because even if these leaves are not totally flat locally, their fold network follows some topological rules: for instance, folds are nearly straight, and thus if two folds originate from the same point, they cannot rejoin somewhere else. Also, each fold, originating either from the petiole (primary folds) or a vein (secondary folds), can only propagates until it reaches the leaf margin and creates either a sinus or a lobe. Some other leaves do not follow these simple rules; their topology has an intrinsic Gaussian curvature, as for Morus platanifolium (as the antifold of Figure 8f'), and their shapes cannot be imitated using a flat sheet of paper. If the external shape of an immature Morus platanifolium leaf is as simple as the "radial folding" case (i.e. the abaxial part corresponds to the protecting veins, and the margin lies on an adaxial plane delimited by a stipules), the folds (Figure 13a) can be curved and thus have non-zero Gaussian curvature, which changes the leaf properties dramatically. If the folds are no longer straight lines, two folds originating from the same point can reconnect elsewhere, and then cancel each other before reaching the edge (Figure 13b1-b2). For this reason, they do not alter the margin. These curved folds enable a new kind of folding such as inverted folds: in this case, a fold along a vein inverts just before the margin and becomes an antifold (Figure $13 \mathrm{c} 1-\mathrm{c} 2-\mathrm{c} 3-\mathrm{c} 4)$.
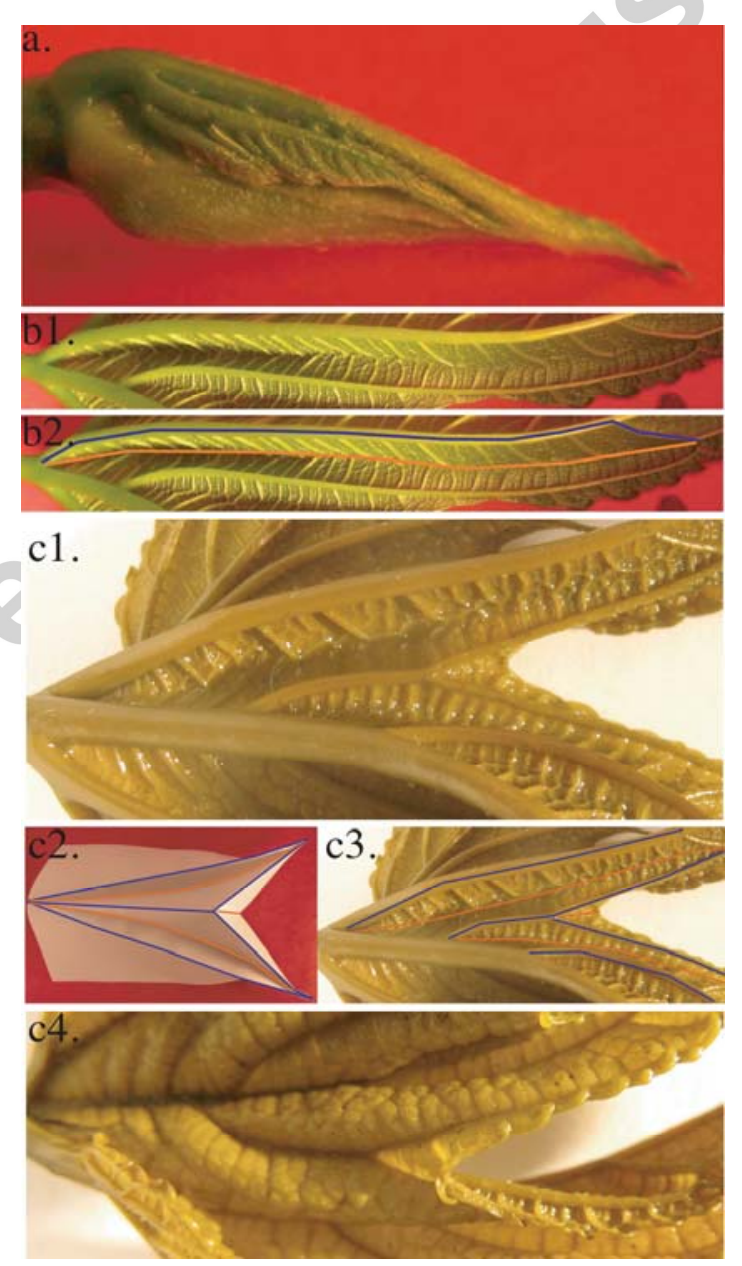

Figure 13. Particular folds of Morus Platanifolium leaves. a: A Morus platanifolium bud. The leaf is folded with its margin on the smaller bud envelope. b1: A Morus platanifolium leaf fold, which ends before the margin of 
the leaf without creating sinus. b2: Sketch of the folds network. (Blue lines correspond to the folds, and orange lines to the antifolds.) c1: A Morus platanifolium leaf fold (along a vein) inverts itself in an antifold just before the leaf margin (abaxial view). c2: Simplified fold network using a sheet of paper. c3: Fold network of c1. c4: Adaxial view of the same inverted fold.

If two curved folds that cancel each other do not directly affect the margin, they have an indirect effect on the shape of the leaf: the antifolds can become the axes of symmetry of the lobes (Figure 14b-b'), and the folds can become the axes of symmetry of the sinuses (Figure 14c-c'). These symmetry rules appear to be the inverse of classical Kirigami (Figure 14d), but they can be simply explained, considering some topological rules. For example, the combination of fold + antifold + fold is equivalent to a simple fold, so that the antifold in between the two folds becomes the axis of symmetry of a lobe (and not a sinus, as is the general Kirigami case, Figure 14b-b'). Similarly, the combination of antifold + fold + antifold is the equivalent of a simple antifold, and the fold between the two antifolds becomes the axis of symmetry of a sinus (and not a lobe, as in the general Kirigami case, Figure $14 c-c ')$.

By taking into account these folds that do not affect the margin but do influence the axes of symmetry (which we call 'mute folds'), we can determine the original folded shape the leaf occupied in the bud. (Figure 15)

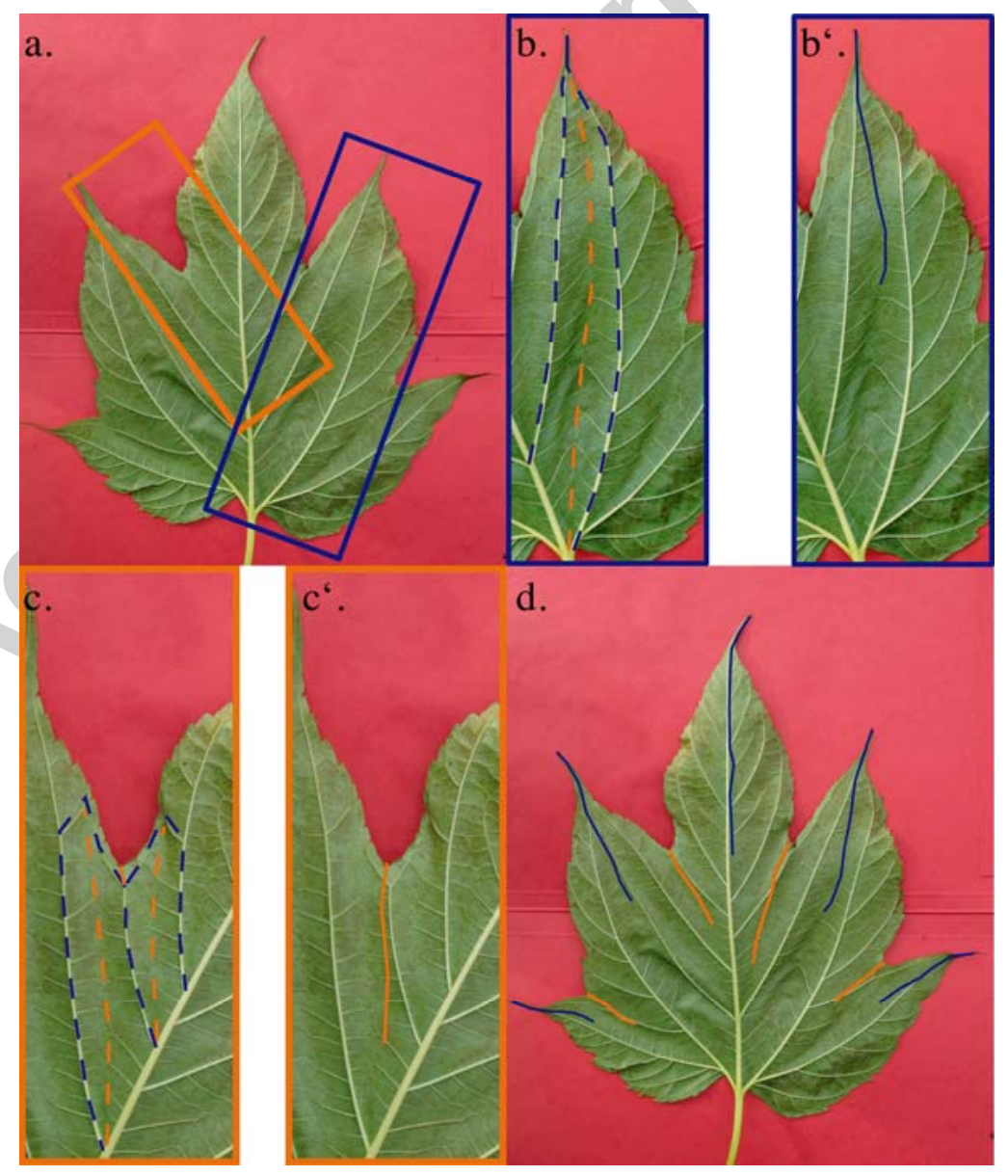

Figure. 14 : Symmetry rules are inverted in non planar leaves. a : A Morus platanifolium leaf. b: The most basal 
fold (dashed blue line) originates at the same point as the antifold (orange dashed line). They merge at the lobe tip without creating either another lobe or another sinus. b': The medial axis of the lobe (blue line) follows the antifold, unlike in the case of Acer pseudoplatanus, whose medial axes follow the fold (Figure 5c). c: The folds (dashed blue line) and the antifolds (orange dashed line) create a complicated network, which is an inverted fold. $c^{\prime}$ : The medial axis of the sinus, when rotated $180^{\circ}$ around the sinus point (orange line) or the symmetric of the medial axis, follows mainly the vein and not the antifold, as in the case of Acer pseudoplatanus (Figure 5b). $d$ : A Morus platanifolium leaf. Symmetry rules are inverted : The symmetry of the medial axis of the sinus (orange line) often corresponds to a vein, and the medial axis of the lobe (blue line) often corresponds to an antifold.

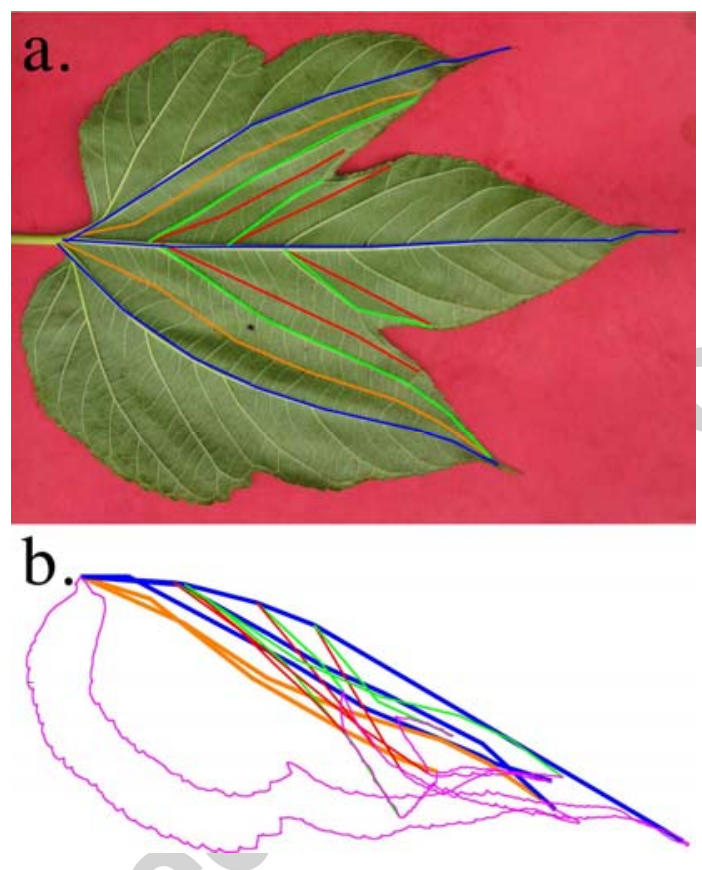

Figure 15. Numerical folding of a Morus Platanifolium leaf. a: A mature leaf of Morus platanifolium. b: Same leaf numerically folded back. The leaf contour is pink. Primary and secondary veins are colored blue and red, respectively. Primary and secondary anti-veins are marked yellow and green, respectively. Synclinal folds run along segments (anti-veins), linking a sinus to the branching point of the two surrounding veins. The thickness of the leaf is not taken into account, and the leaf is folded back onto a plane, approximating the angles as accurately as possible (see Annex).

\section{6 - Adaxial limitation \& self limitation.}

Another particular case is exemplified by Ficus Carica, which is close to Morus platanifolium in the phylogeny. The volume occupied by the leaf in the bud is still a quarter of an ellipsoid, delimited by the previous and next stipules (Figure 16a), which also have curved folds. However, a small detail in the folding leads to a drastic change in their leaf shapes. As in other folded leaves, the central fold develops its lamina partly behind the lateral one and partly in front of the stipules of the smaller bud. At first glance, the whole leaf margin lies on the stipule plane (Figure 16b). But if the central lobe is shifted slightly away from the lateral lobes, the edge of the lobe is shown to run along the vein of the lateral lobe and does not extend up to the stipule plane, except at its end where there is no longer any secondary veins (Figure 16b'). This way of being folded results in the spoon-like shape 
of the lobes. The base of the lobe is thin. because it is limited by the lateral veins (Figure 17). The end of the lobe is large, because it is limited by the bud in front and not by the lateral veins (Figure 16c). This relationship between the base of the lobe and its lateral vein clearly results from regulation, not by chance. This can be demonstrated by the fact that the central and lateral lobes fit together perfectly, while the global shape of the leaf varies strongly in size and number of lobes. Along the stipules, the ratio between the length of the edge of the lobe tip and the rest of the margin can vary from 0.4 to 0.9 (Figure 18a-b). However, the edge of the central lobe always lies along the lateral veins. Do the lateral veins dictate where the edge of the central lobe lies, or vice versa? If we refold the leaf, we see the margins of all lobes almost align with one another, whereas the edge of the base of every lobe (except for the most lateral lobes) lies along the next folded vein (in blue, Figure 16g-h). The numerical folding of a mature leaf (Figure 18b-b') brings the lobe edges close to a plane, even though expansive growth after budding somewhat reduces the fit to a plane in comparison to a young folded leaf (Figure $16 b)$.

The geometry of the packing in the bud leaves its imprint on the mature, unfolded leaf shape. The fig leaf shows that the filling law is widely applicable: it remains true for a curved limitation and a limitation by two distinct objects.

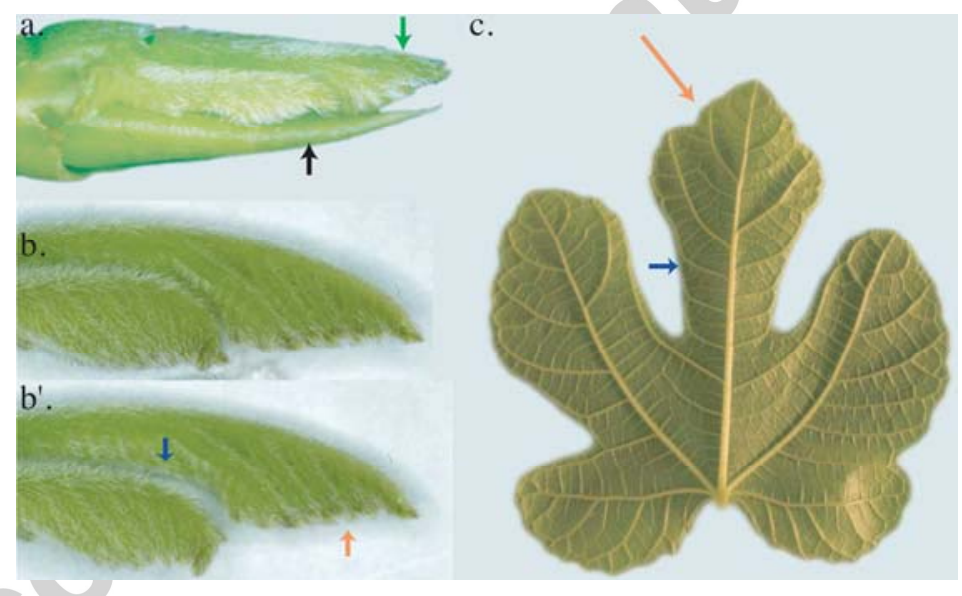

Figure 16. Particularity of Ficus Carica leaf folding. a: A Ficus Carica bud. The leaf (green arrow) is limited by another smaller bud in front (black arrow). b: A single leaf of Ficus Carica. b': The same leaf with the central lobe slightly moved. It reveals that its lower border lies on the lateral lobe (blue arrow), except at the end where it remains along the stipules (orange arrow). c: A mature Ficus Carica leaf, showing the two parts indicated in b', the part limited by the side veins (blue arrow) and the part limited by the stipules (orange arrow). 


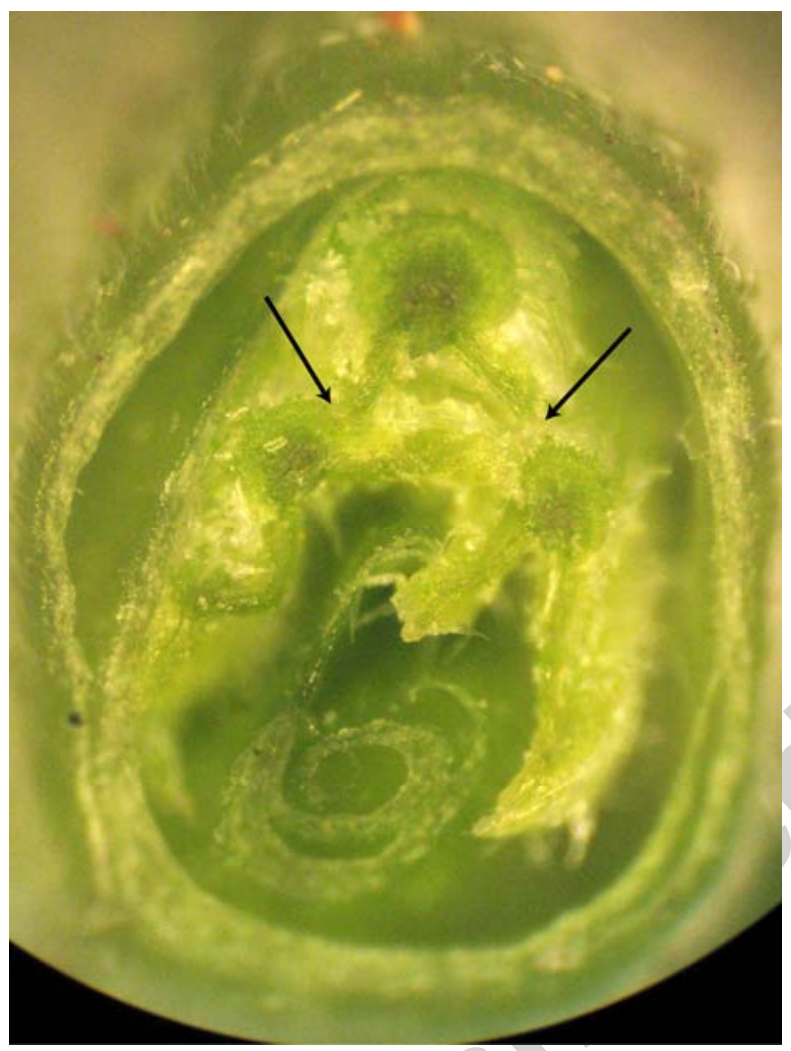

Figure 17: A transverse cut of a Ficus Carica bud. The lamina folded around the central vein ends near the lateral veins, while the lamina of the lateral lobes ends at the lower stipules.

a.

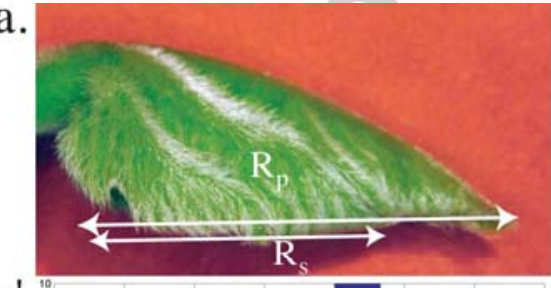

$a^{\prime}$

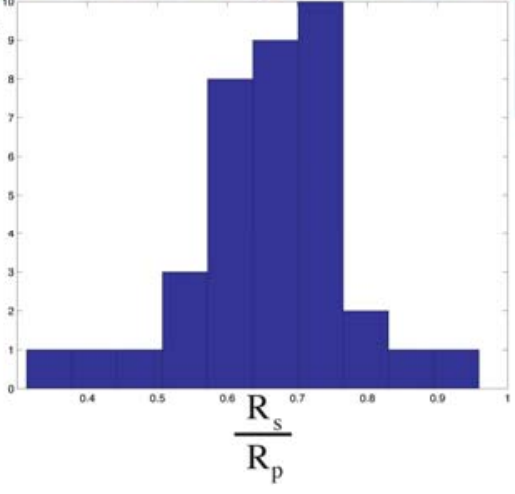

b.

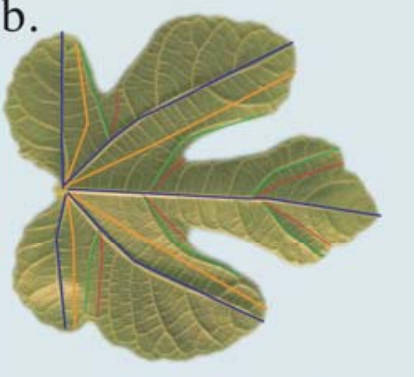

$b^{\prime}$.

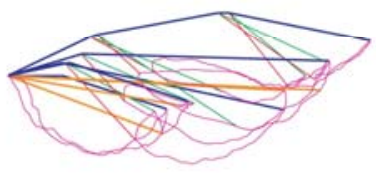

Figure 18. Aspect ratios and folding of Ficus Carica leaf. a: $R_{p}$ is the length of the entire edge of the leaf. $R_{s}$ is the length of the edge, not including the central lobe. a': Histogram of the ratio $R_{p} / R_{s}$, showing large variations. b: A mature leaf of Ficus Carica. Blue, orange, green, and red lines indicate principal folds (lobes), main 
antifolds or valley folds (sinuses), secondary folds (lobes), and secondary antifolds (sinuses), respectively. b': The same leaf folded numerically (see Annex).

\section{7 - Abaxial limitation \& tangential cut}

Suppose a cut delimiting the leaf border is not oriented toward the adaxial plane but toward the abaxial surface. The folds always occur along the veins, which are contiguous with the abaxial envelope. In the previous (i.e. common) cases, it is the adaxial plane that cut the folds transversally (Figure 19a). In the case we consider here, it is the abaxial limitation that cut the folds, but tangentially (Figure 19b). For a purely geometric reason, it considerably changes the geometry of the leaf border. In the case of transverse folding, folds are axes of symmetry of the edge of this filling surface (Figure $\left.19 \mathrm{a}-\mathrm{a}^{\prime}\right)$. But if the cutting direction is tangent to the fold, the fold is no longer an axis of symmetry of this margin (Figure 19b-b').
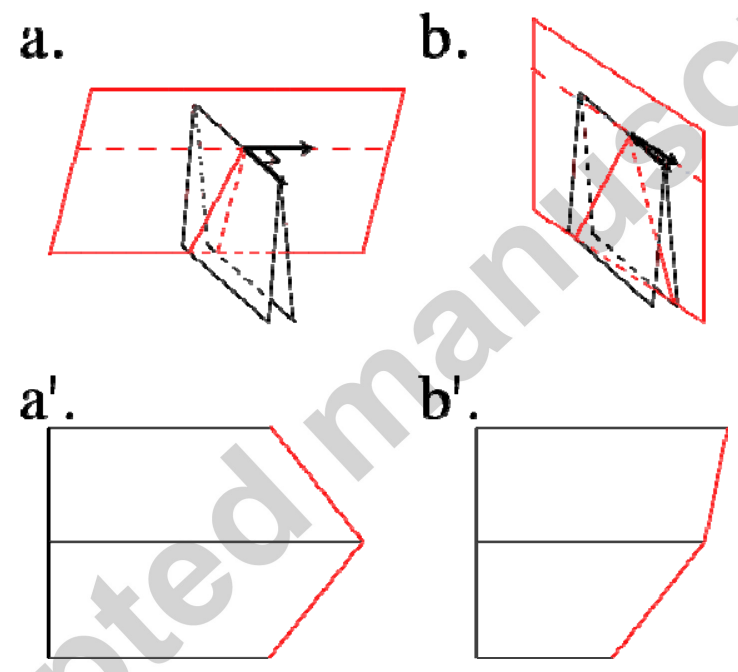

Figure 19. Different geometries of cuts. a : Transverse cut of a fold, the cut plane is orthogonal to the half-planes on the both sides of the fold. a' : Same fold as in a.,unfolded. The fold is the axis of symmetry of the edge (in red). $\mathrm{b}$ : Tangential cut of a fold. The cut plane is nearly tangential to the half-planes on both sides of the fold. b' : Same fold as in b. when unfolded. The fold is no longer the axis of symmetry of the edge (see Annex, 3D Cuts geometry).

It is particularly interesting that this difference between adaxial and abaxial limitations, and thus transverse and tangential cuts, can be obtained within the same species in two different cultivars. An example, among the beechplants, there are the cultivar Rohan obelix (var.) and the normal beech. In the case of Rohan obelix, the cutting plane is adaxial, corresponding to the back of a younger leaf (Figure 20a). Limitation is transverse to the fold, as common otherwise. As a consequence, folds are axes of symmetry of the edge (Figure 20b-c). 
a.

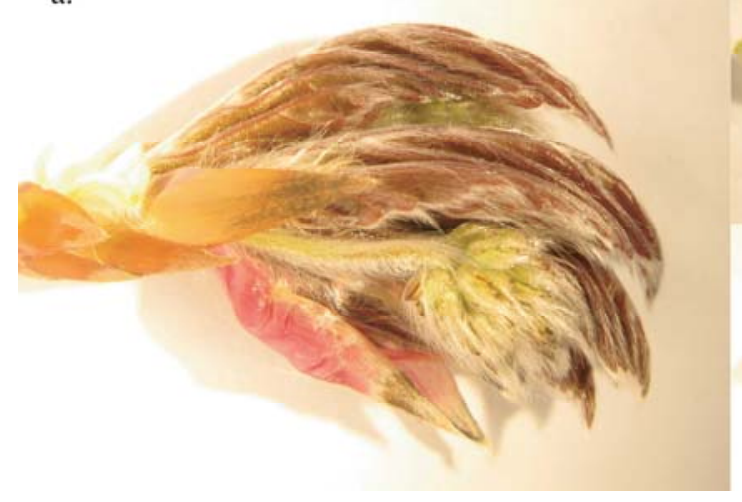

b.

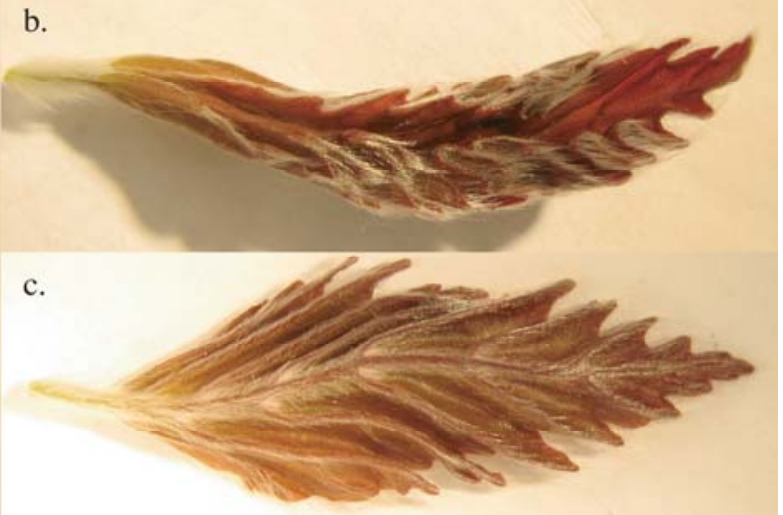

Figure 20. Fagus sylvatica (Rohan obelix var.) bud and leaf. a: A bud with folded leaves. Each leaf margin is folded on the back of the previous leaf, transversaly to the fold. b: Front (adaxial) view of a Fagus sylvatica (Rohan obelix var.) leaf and c: back (abaxial) view. Folds and antifolds are axes of symmetry of the margin.

In case of the classical beech, Fagus sylvatica, the limitation of each leaf is on its abaxial side, contrary to all the other species of kirigami leaves (Figure 21b). The limitation is applied by the envelope of the bud, which is tangential to the folds on the sides (Figure 21a-b). As a consequence, folds are not the axes of symmetry of the margin (Figure 22c). What is unchanged is that even in this configuration, the surface of the leaf still fills the volume at its disposal (Figure 21c).

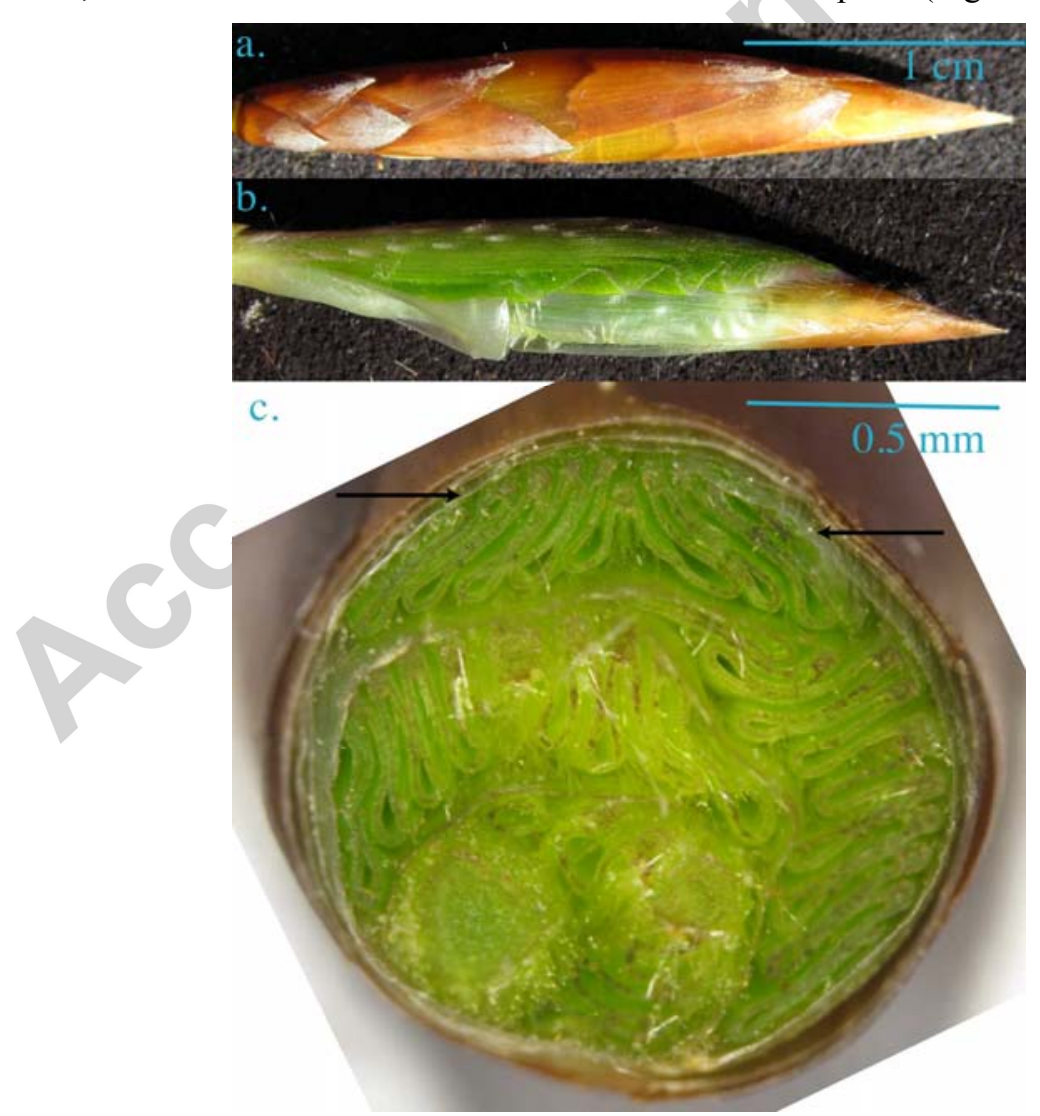

Figure 21. Organization of a wild Fagus sylvatica bud. a: A bud of Fagus sylvatica b: The same bud whithout its upper outer-shell. The fold of the leaves are not axes of symmetry. c: A transversal cut of a bud. The folded leaf margin lies on the abaxial envelope. (black arrows). 
A numerical relationship linking $\alpha$ and $\beta$ (where $\alpha$ is the angle between the two parts of the margin on both sides of the fold, which measures asymmetry, and $\beta$ is the angle between the fold and the limiting surface) can be derived as detailed in Figure 23d-e: $\operatorname{cotan}(\alpha / 2)=\mathrm{h} \tan (\beta) / \mathrm{e}$. To verify that the border of beech leaves follows this relation, we have taken folded beech leaves and measured the angle $\beta$ between the fold and the contour of the folded leaf (which corresponds to the outer-shell) and the angle $\alpha$ of the asymmetry of the cut (Figure 23a-b-c). These angles follow the predicted law (Figure 23f). When $\beta$ is small, the fold is tangent to the limiting surface, and the asymmetry $\alpha$ is large. When $\beta$ is bigger, asymmetry is much smaller.

One also observes this behaviour between the successive secondary folds of the same leaf. The first folds along the central vein are tangential to the outer-shell ( $\beta$ small) and very asymmetric ( $\alpha$ large). On the contrary, the last folds are nearly perpendicular to the outer-shell ( $\beta$ large) and much more symmetric ( $\alpha$ small) (Figure 22c). Repeating these measurements on different leaves, we see that all the measurements align on the same curve (Figure 23g). It means that cutting geometry is conserved among these leaves.

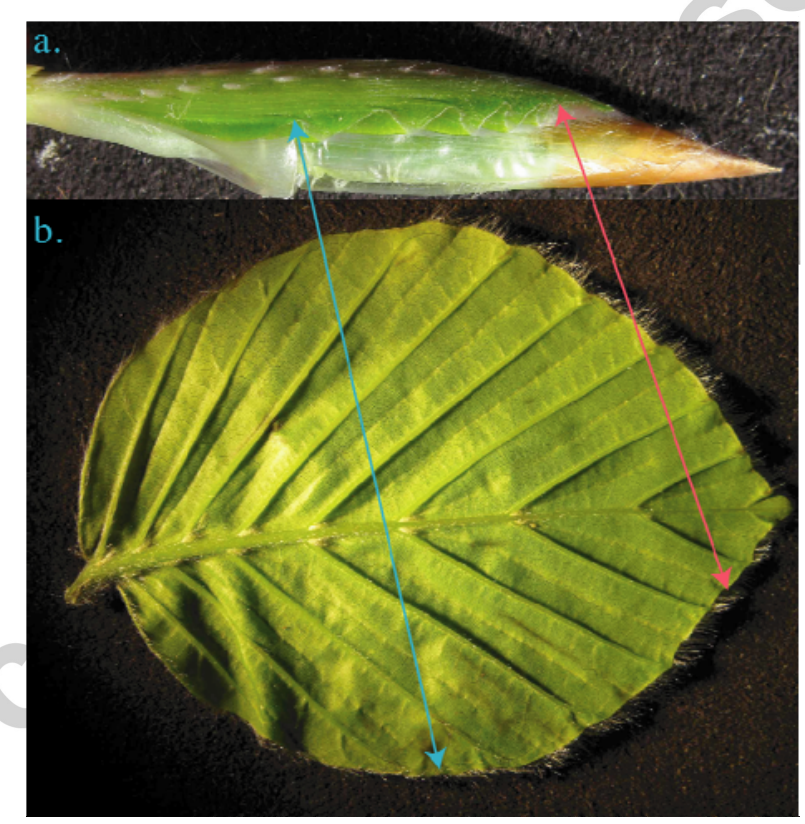

Figure 22. Relation of folding and leaf margin in wild Fagus sylvatica. a. A bud of wild Fagus sylvatica without its upper outer shell. The fold of the leaves are not axes of symmetry. $b$ : A mature leaf. First folds along the central vein are not the axes of symmetry of the margin (blue array), because they correspond to folds tangential to the outer shell. Also, because the folds (which in this case are along the veins) do not lie on top of each other due to the thickness of the veins, the antifold is not equi-distant from the two nearby folds. The last secondary folds along the central vein are the axes of symmetry of the margin, because they correspond to the folds, which are transverse to the outer shell (red array).

Tangential cuts result in leaves whose antivein folds do not always correspond to the sinuses (Figure 24). It depends precisely on where the cut is between two consecutive veins. If the antivein fold-cut is closer to the tip of the first vein, then the antivein fold will yield a sinus, as in the normal case (Figure 
24a-a'). But if the cut is closer to the tip of the second vein, the antivein fold will end in an unusual small bump between the veins (Figure 24b-b'). This observation, in addition to all the preceding calculations, shows that beech leaves are subjected to tangential cutting.

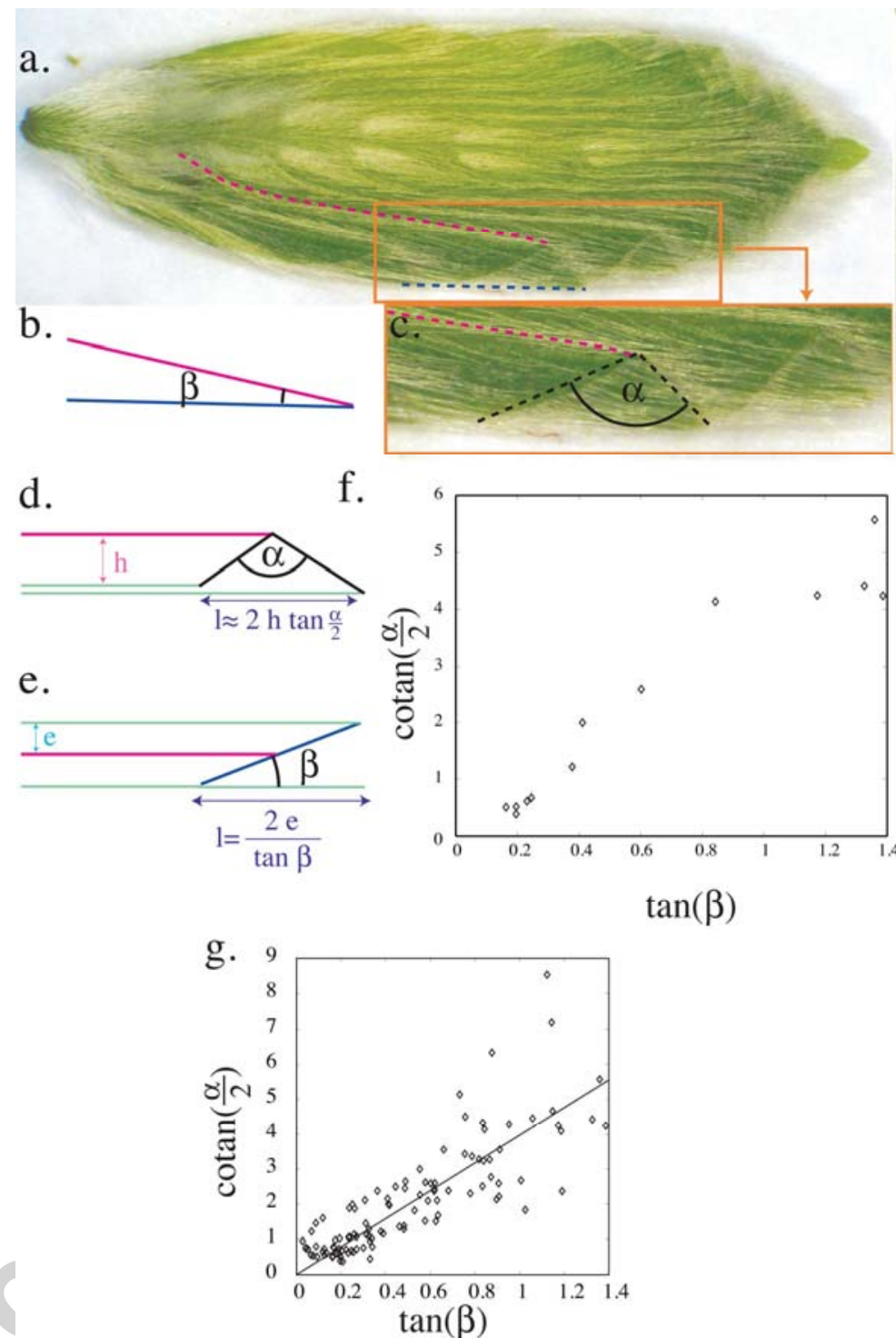

Figure 23. Geometrical relationships of tangential cutting of beech leaves. a: Schema of measurements on the abaxial view of a beech leaf. Magenta dashed line corresponds to the fold, which lies along a vein. Blue dashed line links two consecutive sinuses centered around this vein fold. $b: \beta$ is the angle between the fit of the blue dashed line (represented by a blue continuous line) and the fit of the magenta dashed line (represented by a magenta continuous line). c: The angle $\alpha$ (black) corresponds to the opening of the cut, and to its asymetry with the folds. d: Schema of a lateral view of a fold. First way to calculate the length 1 with the angle of asymetry $\alpha$. e: Schema of an upper view of an unfolded fold. Second way to calculate the length 1 with the border angle $\beta$. $f$ : $\operatorname{cotan}(\alpha / 2)$ as a function of $\tan (\beta)$ for the leaf a. g. $\operatorname{cotan}(\alpha / 2)$ as a function of $\tan (\beta)$ for 8 different leaves.

This example of beech leaves shows that the filling law remains true even in an extreme case where folds are orientated toward the abaxial side and are cut tangentially to the leaf border. The 
difference in the leaf shape and the angle of the cut of the folded leaf, even between two geneticallyclose cultivars, shows that this filling law is stable even when the details of the cutting geometry change drastically.
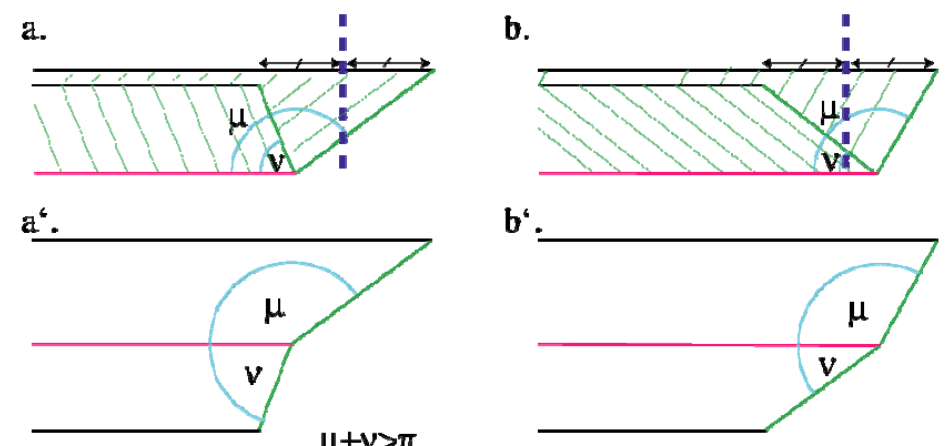

$\mathbf{b}^{\mathbf{2}}$
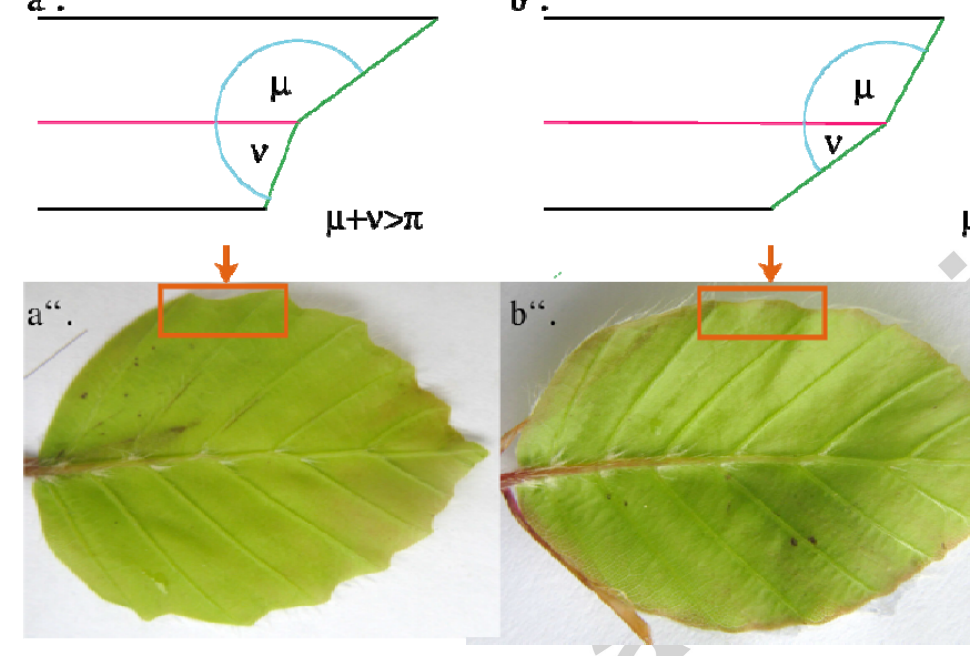

$\mu+v<\pi$

$b^{\prime \prime}$.

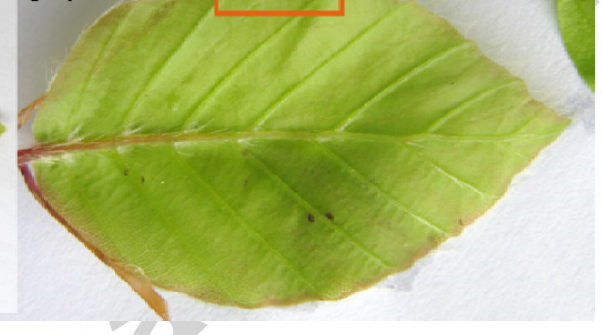

Figure 24. Possible geometries of transverse cuts in beech leaves. The black, red, and green lines correspond to veins, an antivein fold, and the leaf margin, respectively. a: If the antifold's end is closer to the tip of the first vein than to the tip of the second vein, then $\mathrm{a}^{\prime}: \mu+\nu>\pi$, and it will yield a sinus when unfolded. a": Beech leaf whose antifolds become sinuses (orange arrow). b-b'-b": If the antifold's end is closer to the tip of the second vein than to the tip of the first vein, then $\mu+v<\pi$. It will yield a beech leaf whose antivein folds become lobes (orange arrows) (see Annex, 3D Cuts geometry).

\section{3 - Discussion}

\section{1 - Regulation}

Even if leaf shapes are very diverse, a common relation links them to the folding that the leaves experienced when they were young. Leaf shape seems to be the consequence of filling the bud with a certain folding, and not the contrary. Evolution must have acted on this bud packing constrain in generation of the mechanisms of leaf shape regulation. We can thus ask how this packing constrain is encoded in the leaf development. From the point of view of developmental genetics, lobes are reiteration of primordia on the primordia themselves. First they are constituted by an axis, which will give the main vein. The lamina emerges symmetrically from the both side of this axis.

This theory explains why the veins are the axes of symmetry of the lobe margin. But it does not explain why antivein folds are the axes of symmetry for the sinuses. It also doesn't explain why the secondary lobe angles are different, for example, in Tetrapanax papyrifer. The Kirigami rules of folding and cutting seem to override this rule of symmetry of the margin around a central vein, as 
suggested by both the cases of Morus and Fagus. Because of the mute folds, the veins are not the axes of symmetry in Morus platanifolium; because of the tangential cuts, neither the veins nor the antifolds are the axis of symmetry of the Fagus sylvatica leaf margin.

We could, therefore, argue that the Filling law does not come from a pre-controlled mechanism but from a global regulation during development. The lamina could spontaneously develop in symmetry, but its growth is further regulated in the later phases. A possible candidate for integrating the packing is contact regulation by the constraining volume. Contact regulation is common in plant development, such as tree canopy formation (as in the shyness of crown) [Putz et al (1984)] [Prusinkiewicz and Barbier de Reuille (2010)] or the stem expansion [Coutand and Moulia (2000)], although it has been less studied at the level of leaf development [Williams (1975)].

Recently it was shown that mechanics plays a role in primordia development by modulating the direction of growth [Hamant et al (2008)]. The mechanical forces in the Kirigami regulation, however, are not signals to trigger and guide organogenesis, as proposed by Paul Green [Green (1980)] and D'Arcy Thomson [Thomson (1942)]. Rather the mechanical forces generated by physical contacts regulate growth, more precisely halt it, and prevent the increase of the contact pressure. Such contact regulation adds another level at which mechanical forces regulate the development of leaf primordia. This idea was cautiously proposed by Williams [Williams (1975)], and it seems the only reasonable explanation given the tight packing of primordia in the bud, especially because the geometry of the packing correlates with the distinct types of leaf morphology well (see his Figure 4.5.1, 4.5.10 and 5.1 for instance).

Contrary to the mostly studied flat leaves of Arabidopsis thaliana, which grow freely, the leaves studied in this article are stacked and constrained by each other in a three dimensional bud. One conclusion we draw is that folded leaves should not be considered as simple surfaces, but as fully three dimensional objects. Growing folded leaves are then more likely to conflict over the space within the bud, even if there is at least one known example, the cotton ovules, which shows adaptation to avoid such conflicts between organs: the growth of the nucellus could provoke compressive stress in the cotton ovules, but the geometry of integuments insures that the compressive stresses do not collapse the embryo sac, which hangs in a compression-free, open space away from the ovary wall [Lintilhac (1973)].

Our observations hint how the interplay between reiteration of primordia (induction of growth on primordia) and regulation of lobe expansion could work. The secondary lobes in Tetrapanax papyrifer show that the fold angle (angle between the vein and the antifolds) is independent on its position along the main vein, indicating a stereotyped lamina growth, whereas the parameters of the corresponding lobe adapt so that its border end meets the enclosing surface. There seems to be a reiteration of identical folds, but the longitudinal expansion of the lamina, and thus the lobe angle, adapts to the available volume in the environment. The fold seems to be the real unit of reiteration rather than the lobe, whose shape is then determined by the volume limitation, in the Kirigami type 
leaves.

\section{2 - Evolution}

The fact that leaf grows folded could have evolutional significance. The folds of leaves are not random like in expanding poppy petals, but directly related to venation patterns. The first interest is to present the largest veins on the outside. These primary veins are large and robust, mainly composed of spongy tissue which first role is mechanical (parenchyma), but also provide isolation. When damaged, the phloem, which is placed on the abaxial side, induces a rapid chemical response. Thus the veins covering the whole exterior of the leaf primordia provide protection against cold, dryness, and pathogens. The bud structure itself is also aimed to protect the fragile young leaves.

The Filling law also suggests a reason behind the lobed leaf shapes, alternative or complementary to the hypothesis that the leaf teeth and lobes were evolutionary favoured for the efficiency in gas exchange [Royer et al. 2006]. The flat shape of a leaf without lobe is simpler than one with lobes; once folded, on the contrary, the lobed leaf is much simpler and more compact (see Figure 7: compare a folded sheet of paper, $7 \mathrm{a}$, is complicated, while a folded lobed sheet of paper, $7 \mathrm{~b}$, is simpler). The evolutionary reasons of the lobes, thus, might be indirect. It could be an efficient way to develop the largest leaf surface inside a volume with the smallest external surface. In temperate climates, it could be a means to minimize the loss of water during winter and to maximize the photosynthesis when spring comes.

A good indication supporting the notion is the variation of maple leaf shapes, from the almost simple without any lobe to the most elaborate, highly dissected, not only on different species in the tropics or the Northern regions, but even within a single species of maple. Royer et al. [Royer (2009)] found that Acer rubrum leaves form few lobes (3) without any secondary lobes in Florida, but they develop five deep lobes with secondary ones near the Canadian border. A simple interpretation of this result is that the leaf spends more time developing in the bud and created more folds in cold climates, while being able to expand out of the bud sooner in the south, thus making the simpler shape.

This perspective is coherent with the observation that within one species the folded shape of the leaves (with its margin on a plane, or other folding types) is much more conserved than the final unfolded leaf shape, which has a great variability (as we can see in Figure 9 for instance). It seems that the plant regulates the shape of the leaf at this folded stage rather than later. As was the case with phyllotaxis [Douady and Couder (1996))], the particular result may just be a consequence of a dynamical packing problem. The shape of the leaf would just be the consequence of the details of the growth of each bud, their particular geometry and growth history.

This is very different from morphological regulation of the floral organs (e.g. petals), where precise control of the final shape [Rolland-Lagan et al. (2003)] would be important to achieve successful reproduction, especially to attract the pollinator reacting by the shape (and colour and smell, etc.). The function of the leaf is to produce irrigated surface for photosynthesis, relatively independently of its shape. The widespread presences of the Kirigami leaves across the whole 
evolutionary tree of flowering plants also indicate that it is not highly stabilized property, contrary to flower shapes, and also implicate that it is based on basic mechanisms that can be switched on and off easily.

The folding of leaves as a protecting strategy could explain why palmate leaves are predominant in cold temperate regions [Bailey and Sinott (1916)], where such protection is most needed. Some buds have pointed shape (e.g. Ficus Carica and Fagus sylvatica), which might also be a protection against herbivores. Interestingly, these spiky buds correspond to most complicate folding of leaves inside (Figure 25). Leaf shapes could be an indirect consequence of the bud shape, which might be under the pressure by evolutionary selection. For instance, it has been found in tropical rain forest that enrolled monocotyledons buds are less eaten by insects [Grubb et al (2008)].

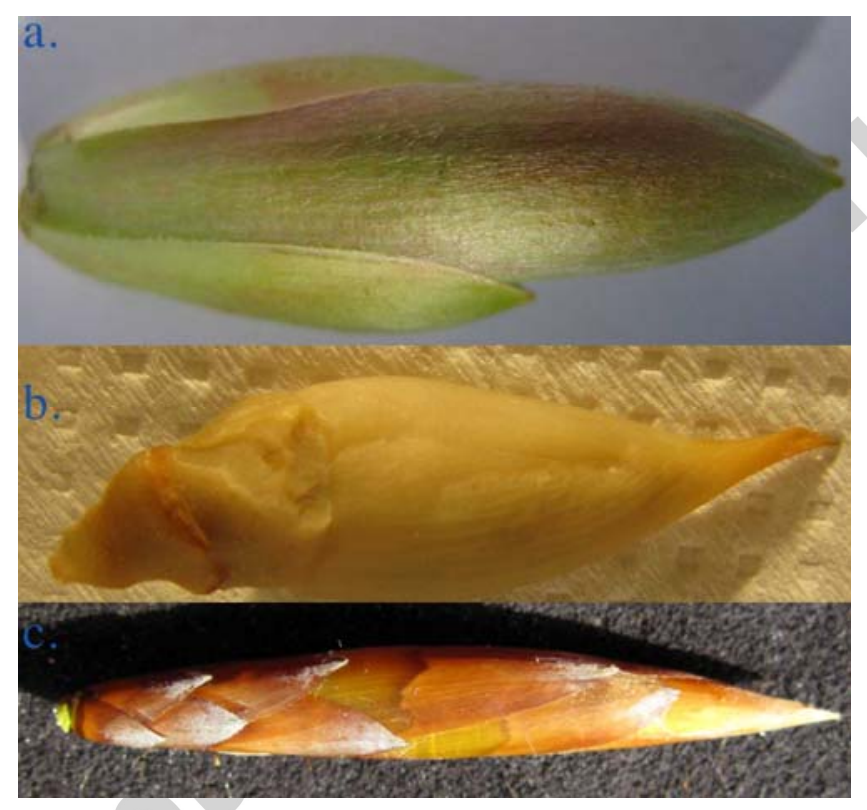

Figure 25. Different bud shapes. a: Acer pseudoplatanus. b: Ficus Carica. c: Fagus sylvatica. The shapes of the bud $\mathrm{b}$ and $\mathrm{c}$ are more acute than the first one.

\section{4 - Conclusion}

We have presented the link between the different kinds of leaf packing inside the bud and their consequences on leaf shape. We have shown how a mild modification in folding can make a drastic change on leaf shape. The main conclusion of this work is that we can predict palmate leaf shapes exactly, if we know how the leaves were folded and oriented inside the bud. By playing with these two parameters, you can create nearly all the leaf shapes observed in nature.

The fact that the bud is always filled with leaves (i.e. the Filling law) implies that the lamina grows until it reaches the border of the constraining volume. Together with the folds, this induces a limitation of the lamina as if it has been cut to fit the volume (the Kirigami Property). This in turn sufficiently determines the unfolded shape. This law holds true in so many diverse geometries as 
presented here, and thus is likely a key rule underlying leaf shape regulation and evolution. All the various leaf shapes can be understood as distinct, efficient ways to fill the available space in the bud with different kind of folds.

One aim of our descriptive botanical work is to formulate hypotheses and questions that can be addressed in developmental biology: for instance, the interplay between "reiteration" and "confinement", as demonstrated by the Tetrapanax papyrifer example. In this example, successive secondary lobes along a main lobe do not have a uniform shape, as the pure reiteration rule would suggest, but they rather adopt a shape which enables them all to be folded along the same line. More generally, the folding and packing regulation are necessary to bridge between the primordial stages of development and the final shapes of the leaves. The fact that the perimeters of these different folded lobes fall at the same border reveals a regulation process that enables precise filling of the bud volume, and we propose that it is a mechanical regulation by contact.

The general theory of leaf shape regulation revealed by our study (the folding of the leaf around the veins, the mechanically sensitive growth of the lamina, and the overall flatness of the leaf), we hope, will inspire further studies, in particular on the underlying molecular mechanisms. The evolutionary aspects would also be interesting to study, in order to identify the selective pressures that shift the types of packing and the corresponding leaf shapes.

Another interesting question which emerges from this study is more mathematical: the non-zero thickness "fold-and-cut" is an unexplored problem. The two different cases of the "auto-cut" fig leaves and the "tangential-cut" beech leaves could be the first step toward a more general geometrical theory inspired by plant development.

\section{Annex - Material and numerical folding methods}

\section{Material}

Acer pseudoplatanus leaves were harvested in October or June in different gardens and forests in and around Paris, France. Tetrapanax papyriferum leaves come from our lab specimens, Pheonix Botanical garden in Nice, and Val Rahmeh Botanical garden in Menton. The buds of Fagus sylvatica, Fagus sylvatica Rohan obelix and Ficus Carica were collected in late spring in the Arboretum of Joinville-le-pont.

\section{Numerical folding method}

Leaves were numerically folded back using these drawing of their veins (synclinal folds), contour and anti-veins (anticlinal folds, Figure 26 a). For instance we took the picture of mature Acer pseudoplatanus or Tetrapanax papyrifer leaves. The main veins and secondary veins were drawn (by hand), acquiring the numerical positions. The contour was also acquired numerically.

The first main veins and anti-veins joined in the same point, at the end of the petiole. For the first refolding of the leaf, we just measured the angles between the successive first segment of veins and 
anti-veins (Figure $26 \mathrm{~b}$ ), and redrew these segments by inverting the sign of one angle on two.

To fold the rest of the vein (i.e after the first segment) we used two different methods, one for the central vein, which is nearly a straight line, and one for the lateral curved one. For the central vein we needed to take into account the secondary folds (Figure $26 \mathrm{Bc}$ ), and for the lateral one the curved antivein (Figure 28 a, a,' b, b', see below).

After having folded these veins and antiveins, we reconstructed the contour as described above. Before folding, we decomposed the initial contour on segments joining two consecutive folds extremities and the normal at this segment (orientation). After folding, we recompose the contour putting each segment on the new position of the end of veins and antiveins.

- Case of secondary folds

To fold the secondary folds there can be a geometrical problem. It is not always possible to keep the actual angles as the resulting folded sheet may not lie flat. The method used to numerically fold the leaves onto a plane keeps at best the fold lengths and the angle values between the folds. Finding close values is a way to project the folding into the plane (or look at it from the side), with minimal distortions. Lets consider the branching detail on figure 26e, sketched in figure 26f. When unfolded, a secondary vein is branching at a primary vein at an angle $\mu$. The primary vein is making an angle $v$ at this branching point. Between these two anticlinal folds, i.e. the secondary and the primary veins, stands a synclinal fold that makes angles $\alpha$ and $\beta$ with them. To these angles $\alpha, \beta, \mu$ and $v$ for the unfolded leaf, correspond the angles $\alpha^{\prime}, \beta^{\prime}, \mu^{\prime}$ and $v^{\prime}$ when the leaf is folded (Figure 26g).

The sum of these angles for the unfolded leaf is:

$\alpha+\beta+v+\mu=2 \pi$

Considering the angle $v^{\prime}$, the sketch of figure $26 \mathrm{~g}$ is in a plane only if (Kobayashi Theorem) :

$v^{\prime}=\mu^{\prime}-\alpha^{\prime}+\beta^{\prime}(1)$

If the sum of folded angles also follows (still on a plane unfolded)

$\alpha^{\prime}+\beta^{\prime}+v^{\prime}+\mu^{\prime}=2 \pi$

equation (1) rewrites:

$v^{\prime}=\pi-\alpha^{\prime}$ 

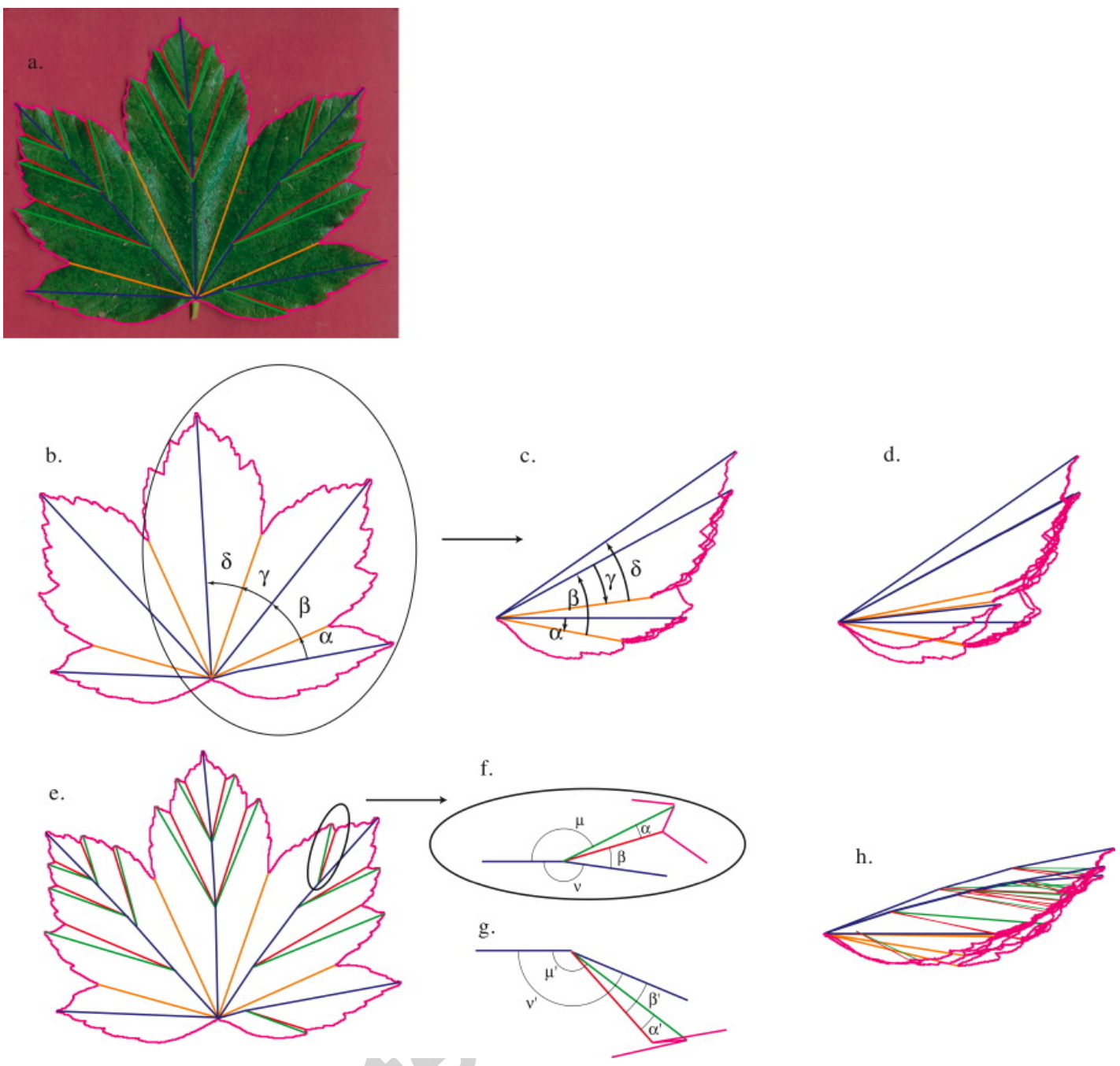

Figure 26 a. On a maple leaf, the first order veins (blue lines) and secondary veins (green lines) are drawn. The first order anti-veins (orange lines), which extend from the intersection of two veins up to the sinus between these veins, and the second order anti-veins (red lines) are also drawn. The contour of the leaf (mangenta line) is numerically detected. b. The result with the contour and only the main veins and anti-veins, and the first angles between them. c. Half part of the precedent sketch once refolded. $\alpha$ and $\gamma$ were reversed, and their respective contour were drawn inversed. d. The refolded leaf using only its main folds. e. Sketch of the leaf with all its folds: secondary one and main one. f. Scheme of a secondary fold, unfolded. g. Folded in a plane. The new angles are obtained as described in the text. h. The whole set of veins and anti-veins is then drawn, with their respective contour, giving the completely refolded leaf.

Folding the branches while keeping to the best the angle $v$ is then minimizing the quantity:

$\left(v^{\prime}-v\right)^{2}+\left(\alpha^{\prime}-\alpha\right)^{2}$

which rewrites

$\left(v^{\prime}-v\right)^{2}+\left(\pi-v^{\prime}-\alpha\right)^{2}$. 
One finds the best $v^{\prime}$ value:

$v^{\prime}=(\pi+v-\alpha) / 2$

In the same way, one finds:

$\alpha^{\prime}=(\pi+\alpha-v) / 2$

$\beta^{\prime}=(\pi+\beta-\mu) / 2$ and

$\mu^{\prime}=(\pi+\mu-\beta) / 2$.

Once the angle corrected, the whole figure of folded veins and antiveins is drawn, and finally the contour is drawn for each vein-antivein segment, reverted if necessary, and with its angular position stretched or compressed if necessary, keeping the distance to the fold center. Usual corrections from the actual angles are around few degrees. For instance, in Figure 6 the corrections are around $1.5^{\circ}$.

\section{- Case of curved folds}

To refold the curved lateral lobes, we have taken their lines of symmetry using the curved adjacent antifold as the axis of symmetry (Figure $27 \mathrm{a}-\mathrm{b}$ ). For this the vein and antifold were cut in small elements, and each vein element is reflected around the closest antifold element. We then stretched the leaf perimeter to fit to the new length of the new vein if necessary. We made the assumption that the curvature due to the secondary folds is already taken in account in the curvature of the vein. We have located the secondary folds at their curvilinear abscissa along the already refolded main vein (Figure 28).

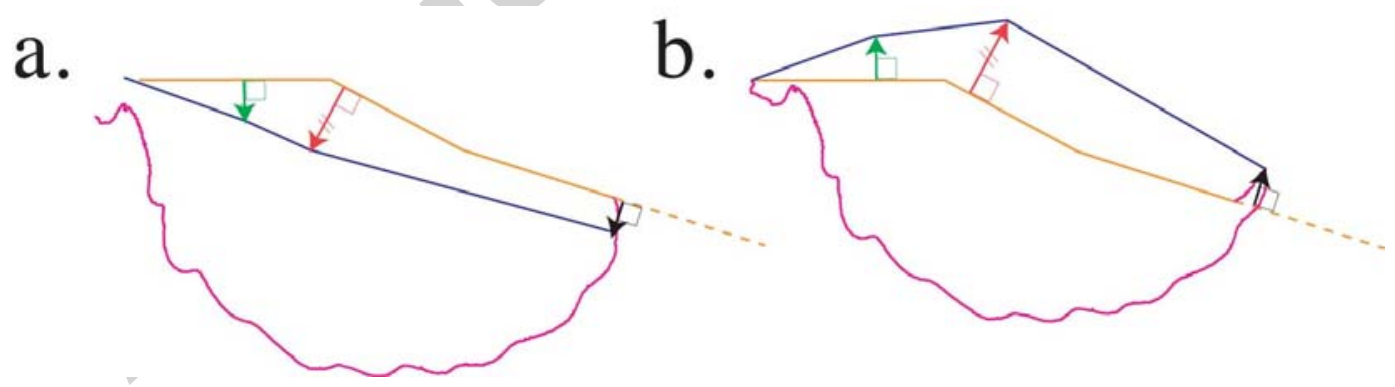

Figure 27: Symmetry around a curved fold. a. An antifold was drawn with an orange line, a fold by a blue one, and the leaf margin by a magenta one. b. After the symmetry of the fold using the antifold as axis of symmetry. 


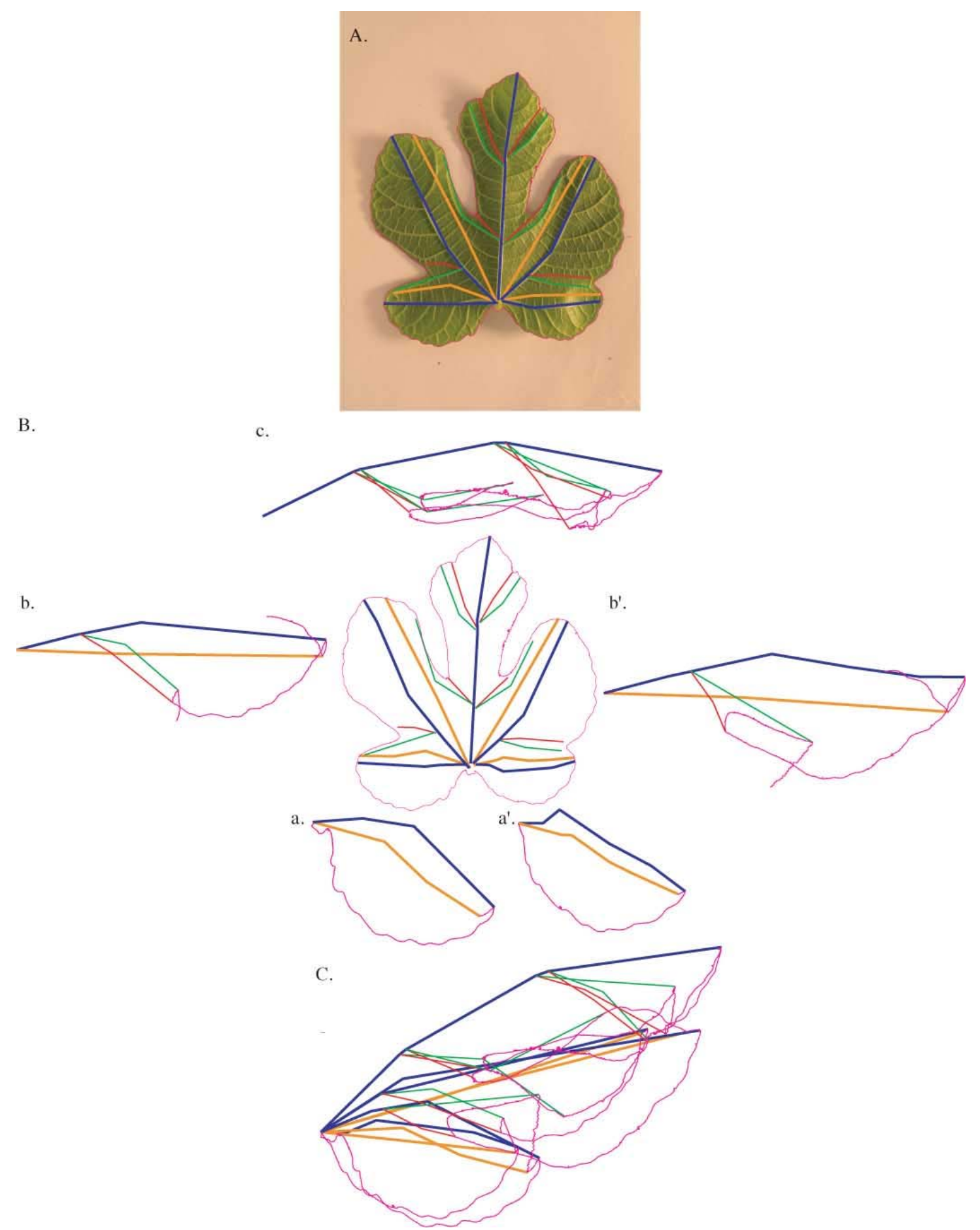

Figure 28: A. Coordinate of folds (along the veins) and antiveins (the zone where secondary veins join) are measured on a Ficus Carica leaf image. B. Folding method. a. We orientate the left lateral lobe symetric (using the antifold as axias) in such way that the first segment of the fold is horizontal. b. We draw the following fold symetric turned with the angle between this fold and the precedent antifold. c. We represent the central lobe with its secondary folds. $a^{\prime}$. b'. Idem of $a$. and $b$ on the right side of the leaf. C. Final result.

\section{D Cuts geometry}



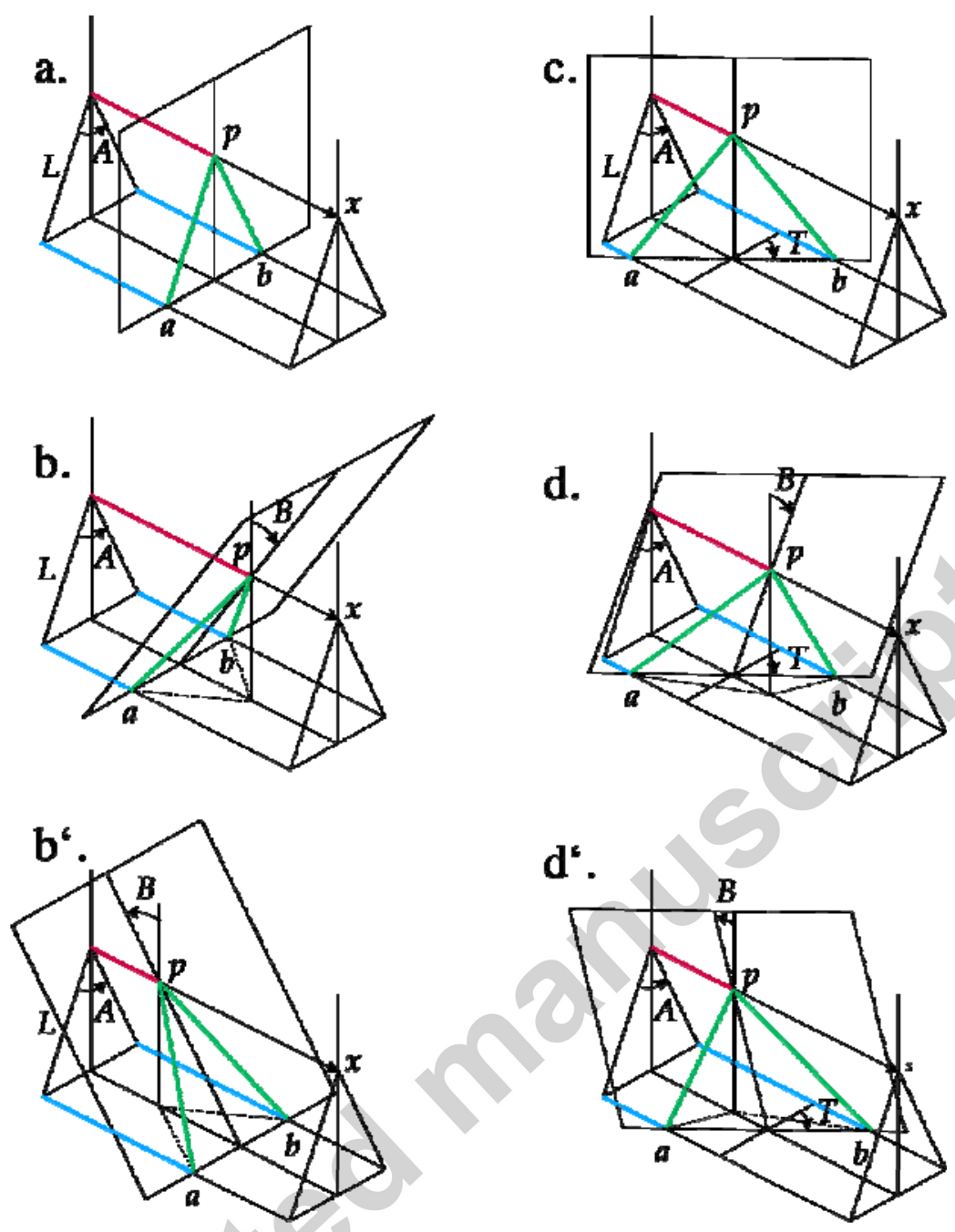

Figure 29 : Geometry of a cut. With a lamina open with and angle A, a vein fold on top (red), and a lamina length on each side L. a : a simple perpendicular cut, leaving a flat perimeter. $b$ : if the cut goes adaxially (inward), with a negative angle B, the positions on the side retract, and the vein fold correspond to the tip of a lobe, as common. b' : if the cut goes abaxially (forward), with a postive B, the side points moves foward on the contrary, leading to the rare case of a vein at a sinus. $\mathrm{d}:$ if the cut is made tranversaly, with an angle $\mathrm{T}$, the points remains aligned on a straight boundary but one moves foward while the other backward. d-d' : when the cut is slented in both ways, then asymatrical cuts corresponding to a point (d) or a sinus (d') can be obtained (as in : Fagus sylvatica Figure 24).

From the figure, one can compute the height below the point $\mathrm{p}$ :

$\mathrm{H}=\mathrm{L} \cos (\mathrm{A} / 2)$

If we take the coordinate of $\mathrm{p}$ along the vein fold $(\mathrm{x})$, as the $\mathrm{x}$ origine $\left(\mathrm{x}_{\mathrm{p}}=0\right)$,

Then for a simple angle B and a null angle T (fig 29 b-b'), we find

$\mathrm{X}_{\mathrm{a}}=\mathrm{L} \cos (\mathrm{A} / 2) \tan (\mathrm{B})$

$\mathrm{x}_{\mathrm{b}}=\mathrm{L} \cos (\mathrm{A} / 2) \tan (\mathrm{B})$

For a simple angle $\mathrm{T}$ and a null angle B (fig $29 \mathrm{~d}$ ), we find

$\mathrm{X}_{\mathrm{a}}=-\mathrm{L} \sin (\mathrm{A} / 2) \tan (\mathrm{T})$ 
$\mathrm{X}_{\mathrm{b}}=+\mathrm{L} \sin (\mathrm{A} / 2) \tan (\mathrm{T})$

and in the general case (fig 29 d-d'), we find :

$\mathrm{X}_{\mathrm{a}}=\mathrm{L} \cos (\mathrm{A} / 2) \tan (\mathrm{B})-\mathrm{L} \sin (\mathrm{A} / 2) \tan (\mathrm{T})$

$\mathrm{X}_{\mathrm{b}}=\mathrm{L} \cos (\mathrm{A} / 2) \tan (\mathrm{B})+\mathrm{L} \sin (\mathrm{A} / 2) \tan (\mathrm{T})$

Thus we see that we can have assymetric perimeters either inward or outward at the vein or antivein fold, as in :

Fagus sylvatica of figure 24 .

\section{Acknowledgment}

We thanck Anna Fumiko Towbridge, Naomi Nakayama, Melanie Pereira for improving greatly the english of the article.

\section{Bibliography}

Adanson, Famille des plantes, chez Vincent à Paris, (1763) - I Partie. Contenant une Préface Iftorik fur l'état ancien et actuel de la Botanik, \& une Téorie de cette Science. $19^{\mathrm{e}}$ Syftême, cclj-cclij.

Bailey, I.W, Sinott, E.W. The climatic distribution of certain type of angiosperm leaves. Am. J. Bot. 3 , 24-39 (1916)

Bell, A. D., Plant Form: an Illustrated guide to flowering plant morphology, Oxford University Press, 342 p. (1991). Ptyxis pp. 36-37

Blein, T. et al A Conserved Molecular Framework for Compound Leaf Development. Science 322, $1835-1839$ (2008).

Clos, D. Monographie de la préfoliation, dans ses rapports avec les divers degrés de classification. Rouget frères et Delahaut, Toulouse (1870).

Coutand, C. , Moulia, B. Biomechanical study of the effect of a controlled bending on tomato stem elongation : local strain sensing and spatial integration of the sigqnal. Journal of Experimental Botany 51 : 352 1825-1842 (2000).

Couturier, E., Courrech du Pont, S., Douady, S., A Global Regulation Inducing the Shape of Growing Folded Leaves. PLoS ONE 4 (11), e7968 (2009).

Cullen, J., A preliminary survey of ptyxis (vernation) in the Angiosperms. Note from the Royal Botanical garden, Edinburgh, 37, 161-214 (1978).

Demaine ED, Demaine ML, Lubiw A. Folding and One Straight Cut Suffice. Proceedings of the 10th Annual ACM-SIAM Symposium on Discrete Algorithms 891-892 (1998).

Dengler, N.G. , Dengler, R.E. , Kaplan, D.R.. The mechanism of plication inception in palm leaves: histogenetic observations on the pinnate leaf of Chrysalidocarpus lutescens. Can. J. Bot. 60: 82-95. (1982).

Dolan, L. \& Poethig, R. Clonal analysis of leaf development in cotton. Am. J. Bot. 85, 315-321. (1998).

Douady, S. \& Couder, Y. Phyllotaxis as a self organizing process-Part I-II-III. J. theor. Biol. 178 255312 (1996)

Esau, K. Anatomy of seed plants (2nd ed.), Wiley (Hoboken, NJ, USA) (1977) 
a Biophysical View. Annual Rev. Plant Physio. Plant Mol. Bio. 31, 51-82

(1980).

Monocot Leaves are

Eaten Less than Dicot Leaves in Tropical Lowland Rain Forests: Correlations with Toughness and Leaf Presentation, Annals of Botany 101: 1379-1389 (2008)

Hamant, O. , Heisler, M. G. , Jönsson, H. , Krupinsky, P. , Uytteval, M. , Bokov, P. , Corson, F. , Sahlin, P. , Boudaoud. , A. , Meyerovitz, E. M. , Couder, Y. , Traas, J. Developmental Patterning by 322: $1650-1655$ (2008)

Hofmeister, W., Allgemeine Morphologie der Gewächse. In: W. Hofmeister (ed.): Handbuch der Physiologischen Botanik I-2. W. Engelmann, Leipzig. (1868).

Jussieu, Adrien de, Cours Elémentaire d'Historie Naturelle: Botanique, Masson, Langlois \& Leclercq ed., Paris, (1839).

Kaplan, D.R., Dengler N.G., Dengler, R.E. The mechanism of plication inception in palm leaves problem and developmental morphology, Can. J. Bot., 60, 2939-2975 (1982).

Kaplan, D.R., Dengler N.G., Dengler, R.E. The mechanism of plication inception in palm leaves histogenic observations of the palmate leaf of Rhapis Excelsa, Can. J. Bot., 60, 2999-3016 (1982).

Kobayashi, H., Kresling, B. \& Vincent, J.F.V. The geometry of unfolding tree leaves. Proc. R. Soc. Lond. B 265, 147-154 (1998).

Kobayashi, H, Daimaruya, M and Vincent, JI .V. Folding/ Unfolding Manner of Tree Leaves as a Deployable Structure. IUTAM-IASS Symposium on Deployable Structures: Theory and applications, Pellegrino, Guest ed. 211-220 (2000).

Lamarck, J.-B. De, Candolle, A.P. De, Flore Française, 3eme ed. Dresay, Paris, 1815

Linthilac, P. Differentiation, Organogenesis, and the Tectonics of Cell Wall Orientation. II. Separation of Stresses in a Two-Dimensional. Am. J. Bot. 61, 135-140 (1974)

Mahadevan, L; Rica, S, Self-organized origami, Science, 307 , 1740-1740, (2005).

Putz, F. E., Parker, G. G. and Archibald, R. M. Mechanical Abrasion and Intercrown Spacing. American Midland Naturalist 112:24-28. (1984)

Prusinkiewicz, P., Barbier de Reuille, P. Constraints of space in plant development. Journal of Experimental Botany, 61: 2117-2129. (2010)

Rolland-Lagan, A. G., Bangham, A. J. Coen, E. Growth dynamics underlying petal shape and assymmetry. Nature 422: 161-163 (2003).

Royer, D.L., Wilf, P., Why do toothed leaves correlate with cold climates? Gas exchange at leaf margins provides new insights into a classic paleotemperature proxy. International Journal of Plant Sciences 167: 11-18 (2006).

Royer, D.L., Meyerson, L.A., Robertson, K.M., Adams, J.M., Phenotypic Plasticity of Leaf Shape along a Temperature Gradient in Acer rubrum PLoS ONE 4(10): e7653 (2009) Thompson, D.A.W., (1942) On Growth and Form, 2nd edn Cambridge: Cambridge Univ. Press (2 t); Reprint: (1992) Dover. 


\section{ACCEPTED MANUSCRIPT}

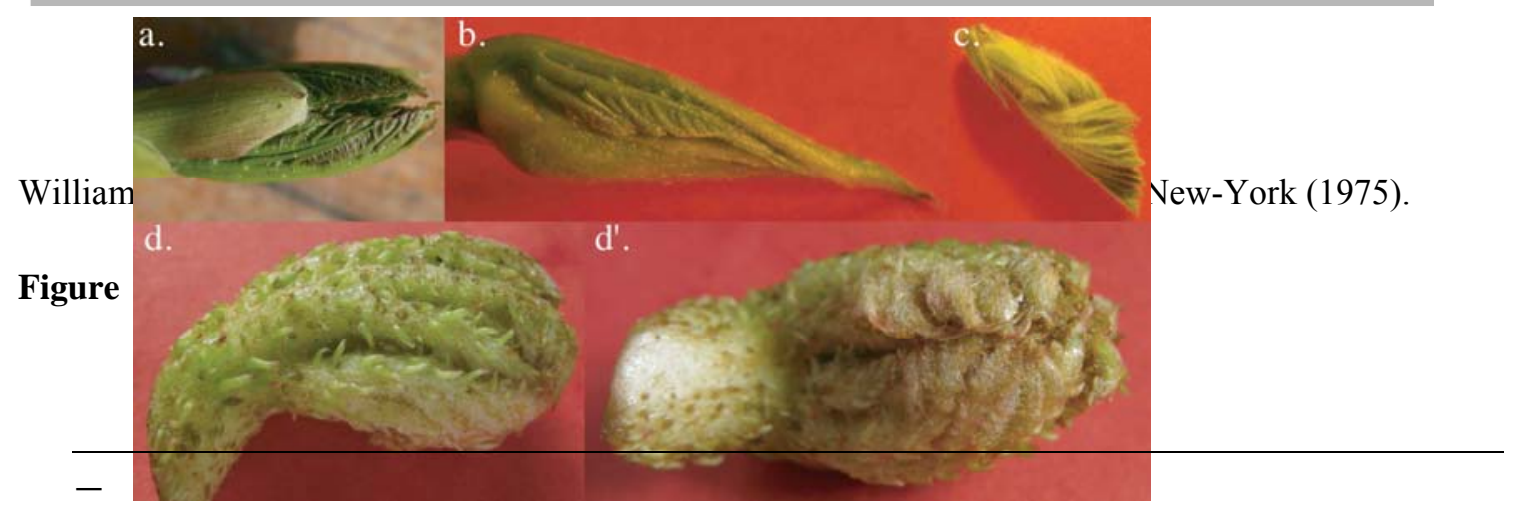

Figure 1: Similar structures in different kinds of buds. The external part is always constituted by veins and the lamina is folded inside. a. An Acer pseudoplatanus bud. The folded leaf margin lays on a plane against the opposite leaf margin. b. A Morus platanifolium bud. The folded leaf margin lays on a plane against the stipules, which envelopes a smaller bud. c. A Pelargonium cuculatum bud. The folded leaf margin lays on a plane against the lower part of an older leaf. d. Gunera manicata view of the leaf. d'. Front view of the same leaf. The leaf constitutes its own bud, and the lamina is delimited by the central axis (as seen in Figure 2 c).
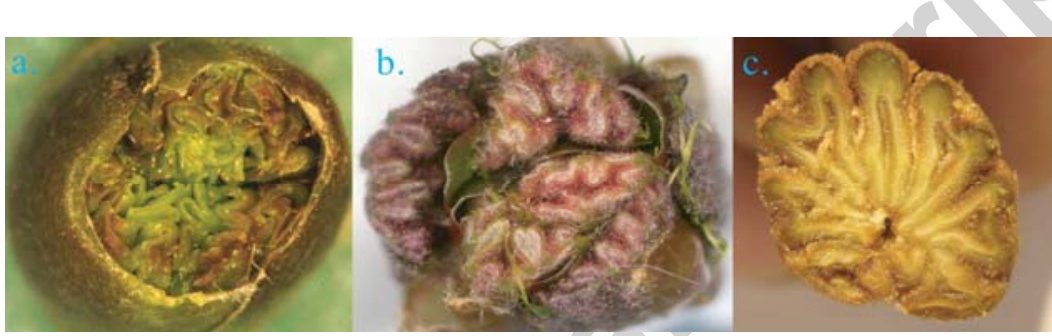

Figure 2: Three cuts of the three different bud kinds : a perfect and rational filling of the bud. a. Opposite phyllotaxy, Acer pseudoplatanus. b. Spiral phyllotaxy, Quercus Rubra. c. Autoclosing leaf, Tetrapanax papyrifer.
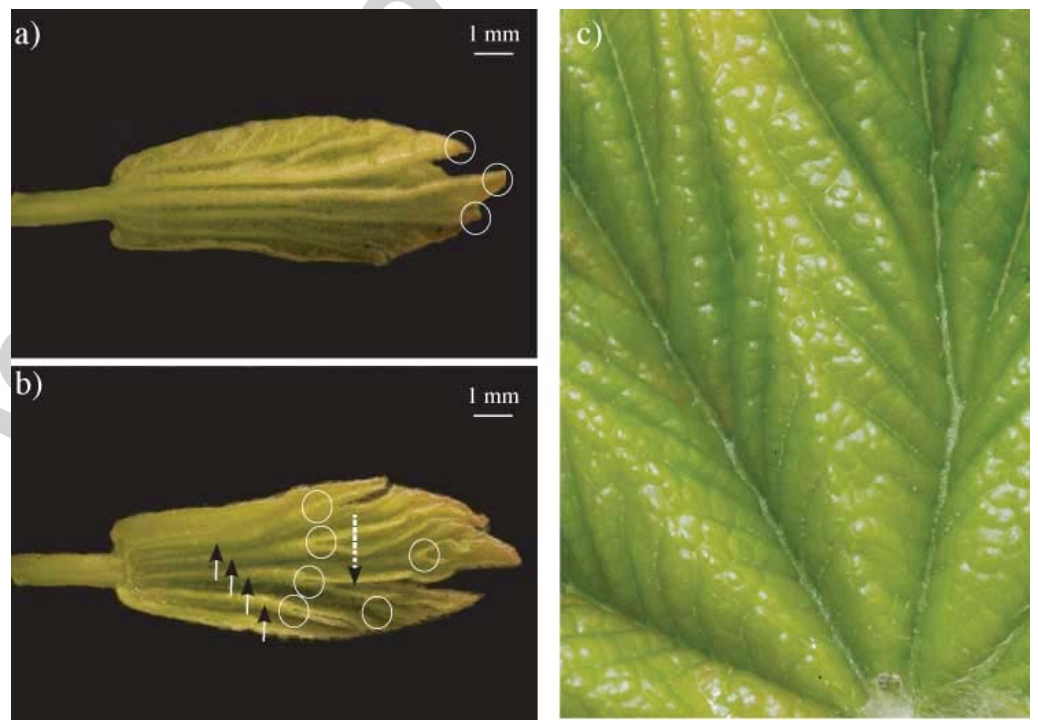

Figure 3: Folded immature leaf extracted from a bud of Acer campestre. a. The abaxial side shows the folds running along veins and ending at peaks (circles). b. The adaxial side shows the lamina folds (arrows) running along (immaterial) anti-veins and ending at sinuses (circles). Peaks and sinuses lay in the contact plane of the pair of leaves (Figure 1), but while peaks are at the extreme of this contact surface, sinuses are positioned inside. c. Upward rising antifolds of Acer pseudoplatanus between the main veins. Only the last order veins, emanating 
from surrounding main veins, join along the antifold.

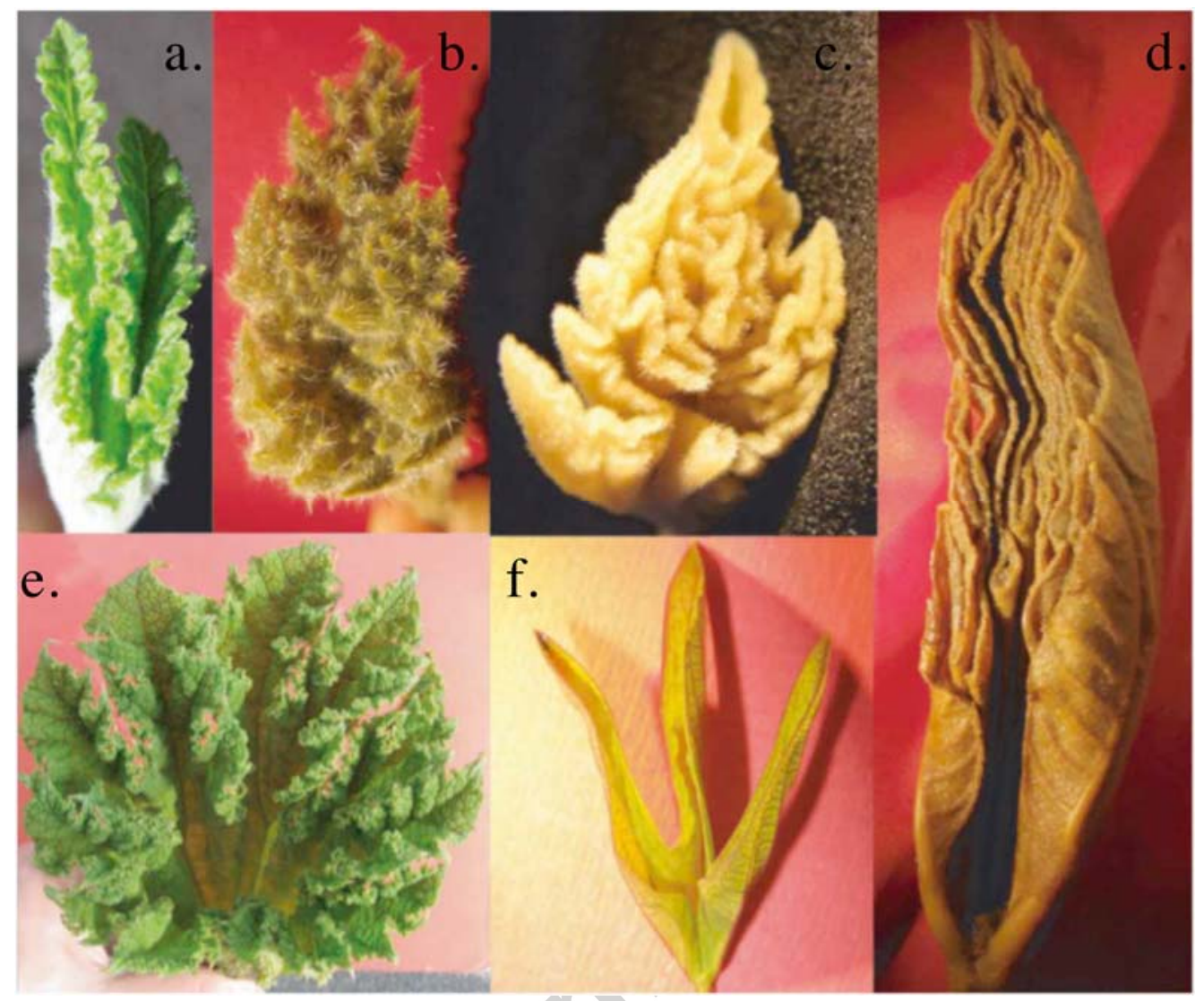

Figure 4: Front view of different folded-and-cut leaves . a. Ribes Nigrum. b. Pelargonium cuculatum. c. Malva Sylvestris. d. Tetrapanax papyrifer. e. Gunnera manicata. f. Passiflora. They are of different phyllogenetical origins: a belongs to Saxifragales, b to Geraniales, c to Sapindales, $\mathrm{d}$ to Apiales, e to Gunnerales, and $\mathrm{f}$ to Malpighiales.

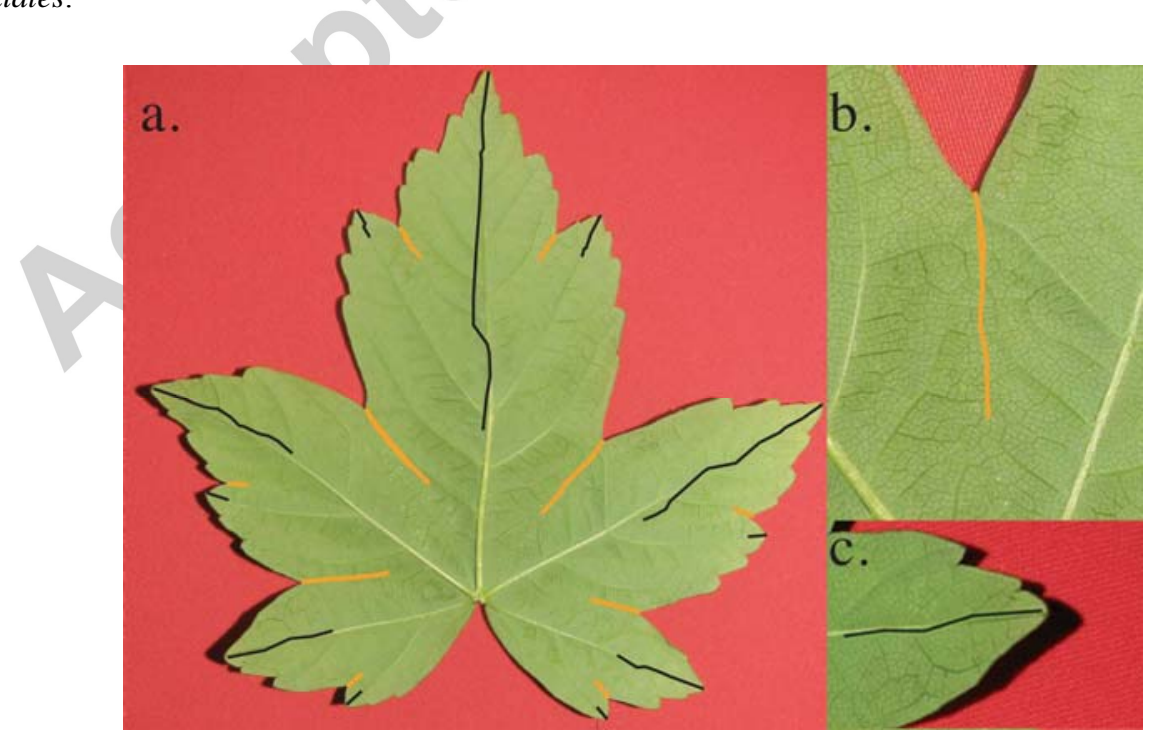

Figure 5: a. An Acer pseudoplatanus leaf with symmetry lines drawn. Black lines marks the medial axes of the lobes, derived from the perimeter. Orange lines are the symmetries around the sinus minmum of the medial axis of the sinus. $\mathrm{b}$. The orange line corresponds to a lamina, or an antivein, fold. $\mathrm{c}$. The black line corresponds to a 
vein fold. The black lines are not exactly on the veins, because they were determined from the leaf boundaries, and thus were influenced by the secondary perimeter variations.

a)
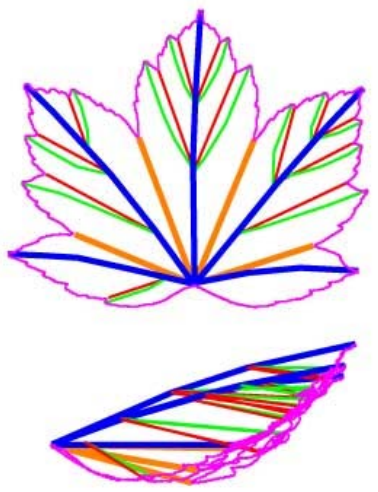

c)

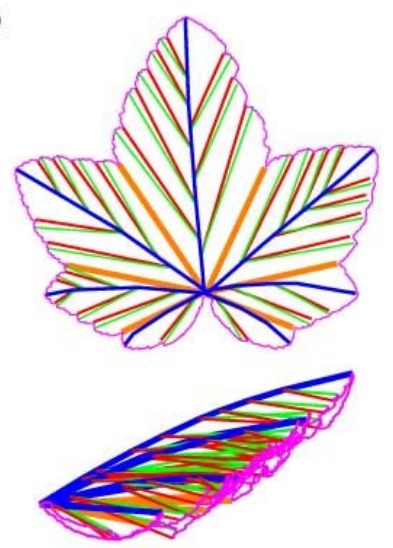

b)
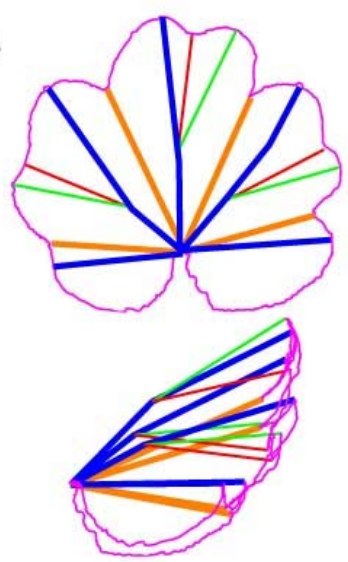

d)

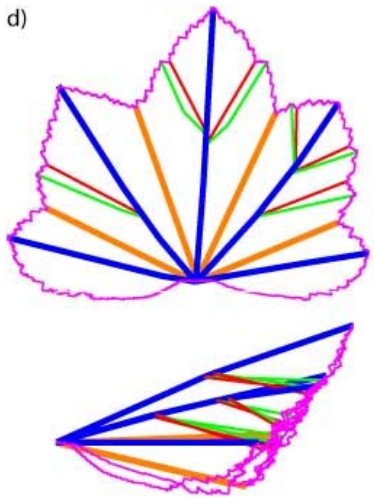

Figure 6: Numerical folding of several species. a. Acer pseudoplatanus. b. Gunera manicata. c. Ribes Nigrum. d. Malva sylvatica. Note that the perimeter refolds on a single line even for asymmetric leaves, as in $\mathrm{d}$. See Annex for the method of numerical folding.

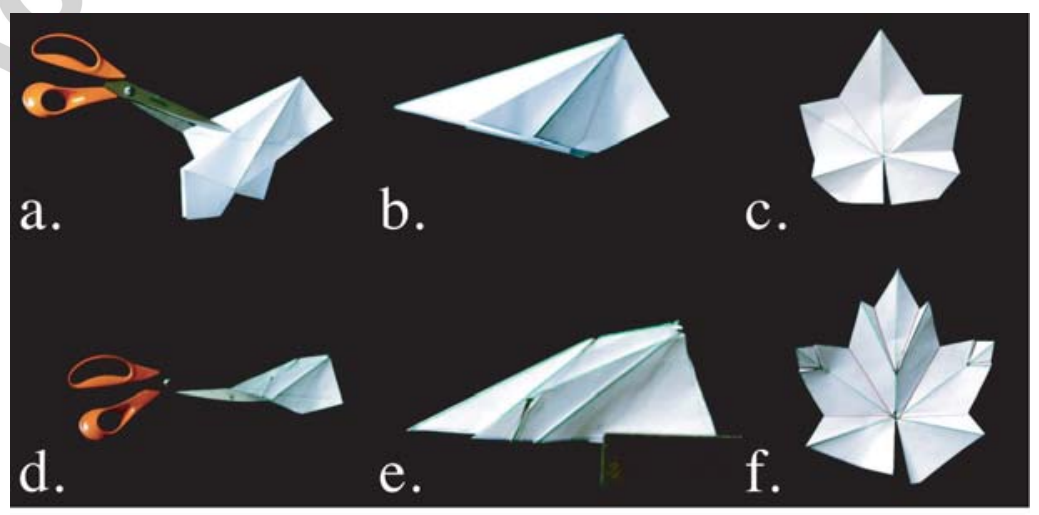

Figure 7: Examples of cut, folded paper in the shape of a leaf (Kirigami -'(folded)-cut paper' in japanese) a. A rectangular sheet of paper with five folds originating from the same point (petiole) is then cut in a straight line 
with scissors. The folded margin is complicated. b. Same folded sheet of paper, cut. The folded margin lays on a simple surface. c. Same sheet, unfolded. Folds correspond to sinuses and lobes. d. Same sheet folded with secondary folds. e. Same sheet, cut f. Same sheet, unfolded. Secondary folds correspond to secondary lobes.

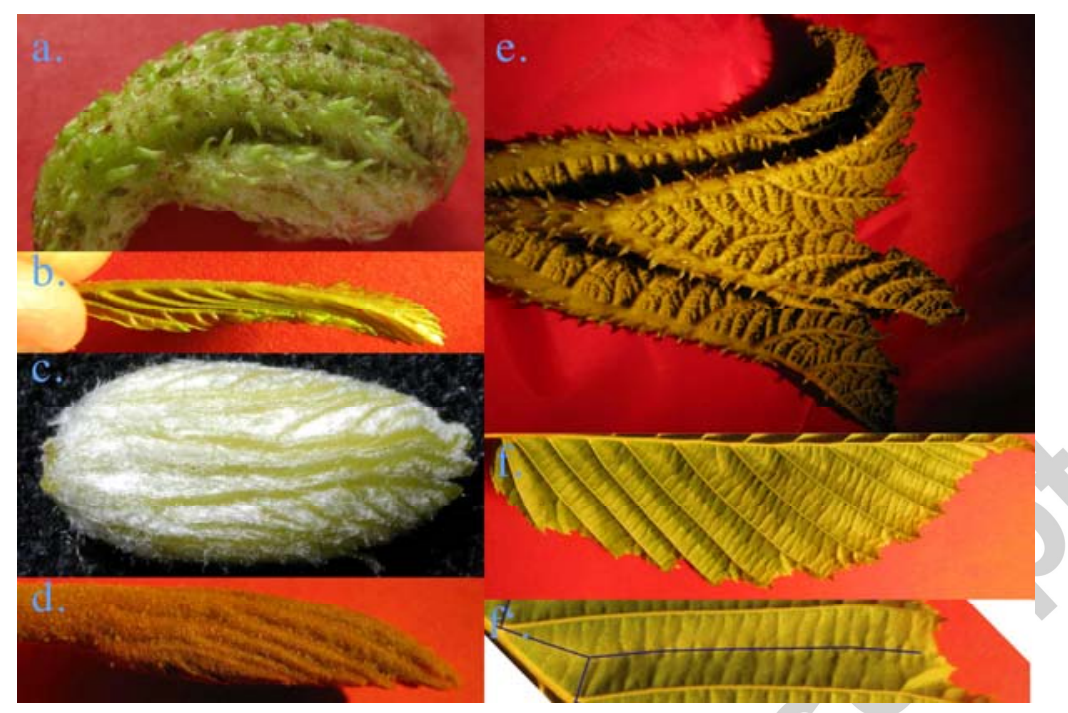

Figure 8: Veins are on the outside of the folded leaf, protecting the lamina with antivein folds inside. a. Gunnera manicata. b. Carpinus betulus. c. Acer pseudoplatanus. d. Tetrapanax papyrifer. e. As the antifold folds a vein on its neighbouring vein, and the lamina is locally flat, the antifold is situated at the bisector of two adjacent veins. f. f'. For the same reason, the antivein fold which is situated between three veins, is constituted by three pieces of bisectors.

aI.
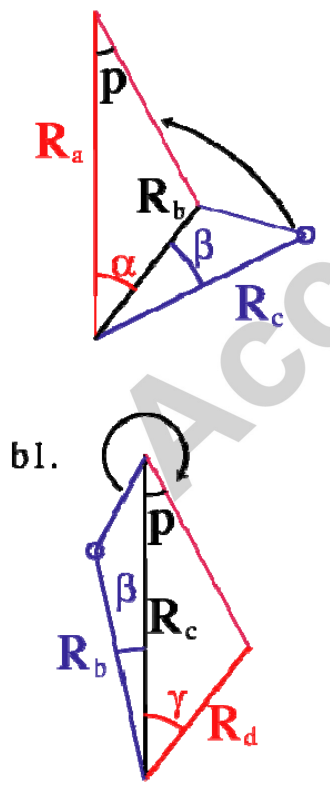

a2.

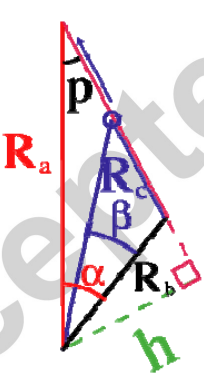

b2.

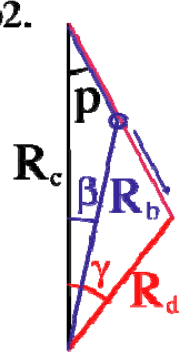

a3.

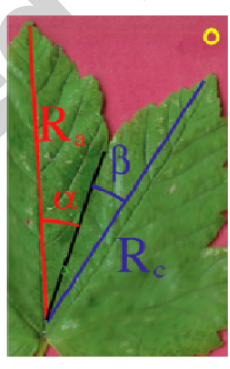

a4.

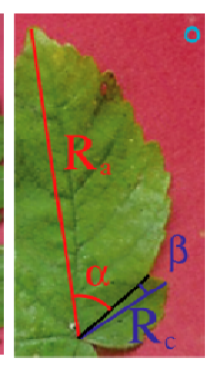

b3.

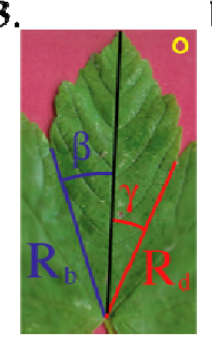

b4.

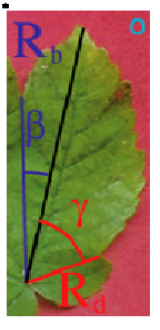

a5.
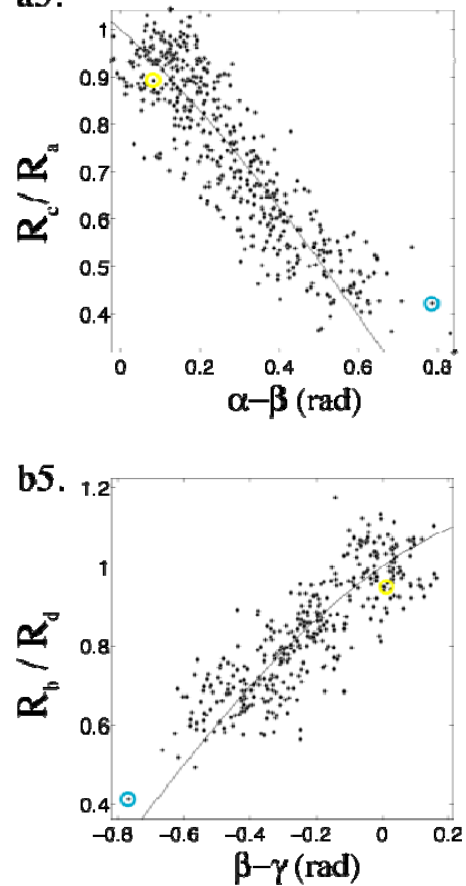

Figure 9: Geometric relationships between two successive lobes and sinuses due to the Kirigami property. a1:

Two consecutive primary lobes have veins of lengths $R_{a}$ and $R_{c}$. They respectively make angles $\alpha$ and $\beta$ with the anti-vein between them, of length $R_{b}$. b1: The vein of length $R_{c}$ is surrounded by two anti-veins of lengths $R_{b}$ and 
$R_{d}$. These are respectively making angles $\beta$ and $\gamma$ with the vein. a2: From the different expressions of the height $\mathrm{h}$, one can derive quantitative relationships between these elements (see text). a3-4 : two extreme examples from real leaves. a3: When lobes have equal length the anti-vein is then the bisector $(\alpha \sim \beta)$. a4: When the smaller lobe is close to the anti-vein $(\beta<<\alpha, a 4)$, its length is that of the antivein. In general, the antivein is always closer to the smaller lobe. a5: Quantitative relationship between two successive lobes and sinuses due tothe Kirigami property, for 121 Acer pseudoplatanus (sycomore) leaves. Length ratio $\left(\mathrm{R}_{\mathrm{a}} / \mathrm{R}_{\mathrm{c}}\right)$ of two consecutive main veins in function of the difference $(\alpha-\beta)$ between the angles they make with the anti-vein. b: same figure for two antiveins on the side of the central vein lobe. b5 : Length ratio $\left(R_{b} / R_{d}\right)$ of two consecutive main anti-veins in function of the difference $(\beta-\gamma)$ between the angles they make with the vein. When anti-veins are equal in length the vein is then the bisector $(\beta=\gamma, \mathrm{b} 3)$. The vein always runs closer to the smaller anti-vein (b4).

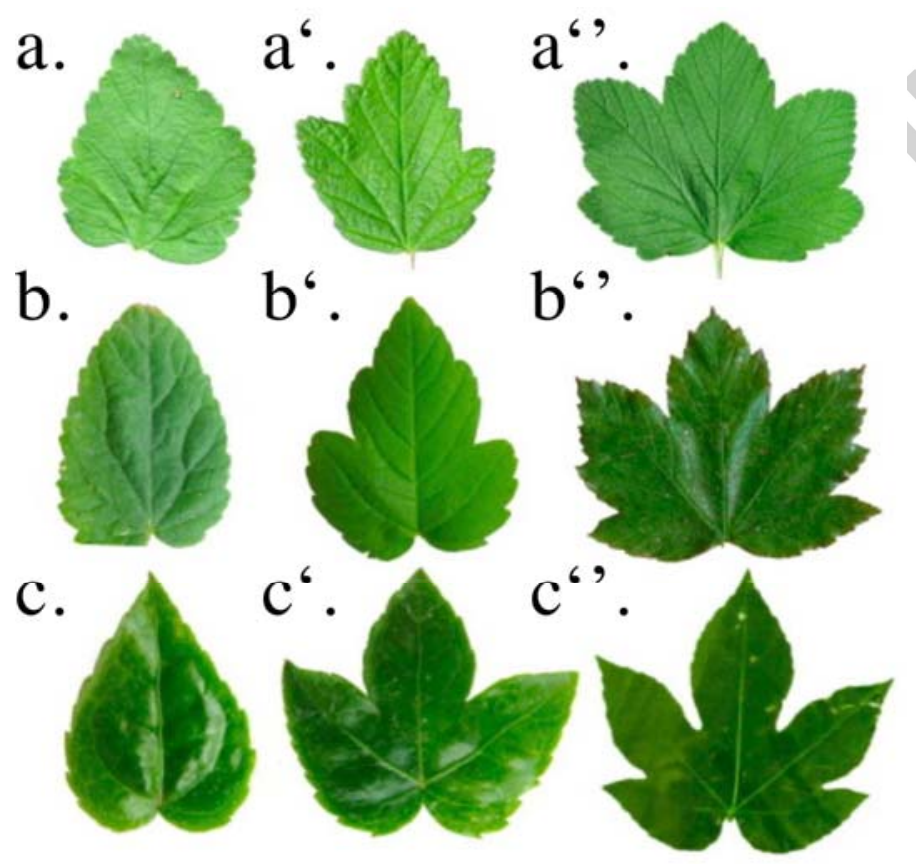

Figure 10: Fold-and-cut leaves from different phylogenetic origins have the similar traits of leaf shapes. a-a": Ribes Nigrum leaves, Saxifragales. b-b": Acer pseudoplatanus leaves, Sapindales. c-c": Fatsia japonica leaves, Apiales. 


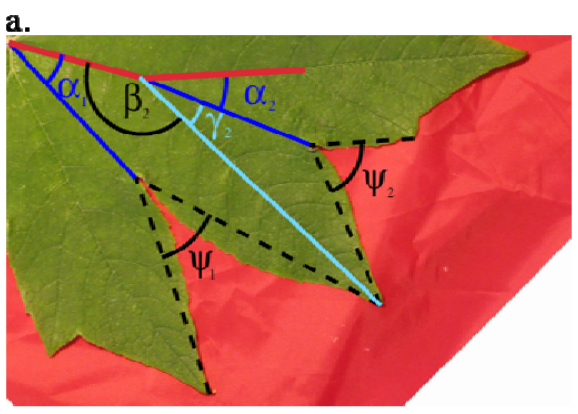

b.

c.
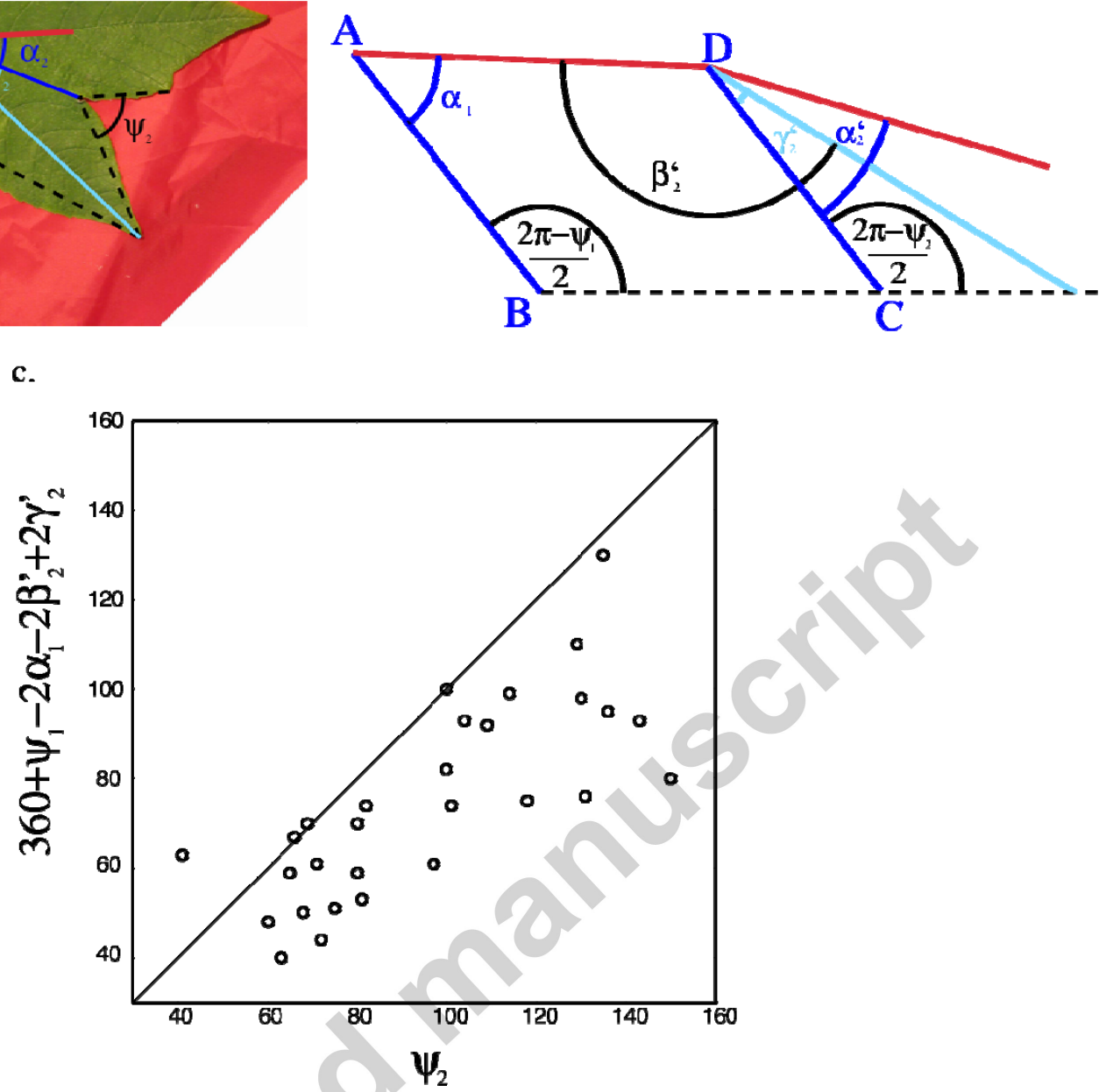

Figure 11. Quantitative relationship between successive secondary folds. a: A lobe of a Tetrapanax papyrifer leaf. The red line corresponds to the main vein. The cyan line corresponds to the secondary vein. The blue line corresponds to the antifold. Notation for the measured angle. $\alpha_{1}, \varphi_{1}, \alpha_{2}, \beta_{2}, \gamma_{2}$ and $\varphi_{2}$. b: The same lobe, folded. $\beta_{2}^{\prime}$ and $\gamma_{2}^{\prime}$ are the nearest angle from $\beta_{2}, \gamma_{2}$ that you can fold in a plane, which is why we used them to make the prediction. c: Data obtained by measurement on five leaves of Tetrapanax papyrifer, with $\psi_{2}$ on the abscissa and the prediction made by assuming that all the lobe refolds along the same line on the ordinate. 
a.

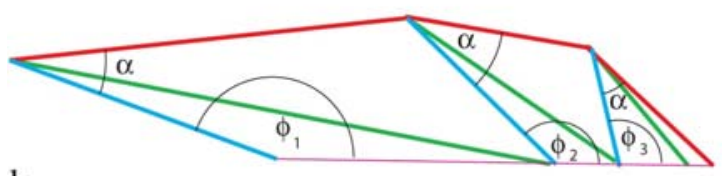

b.
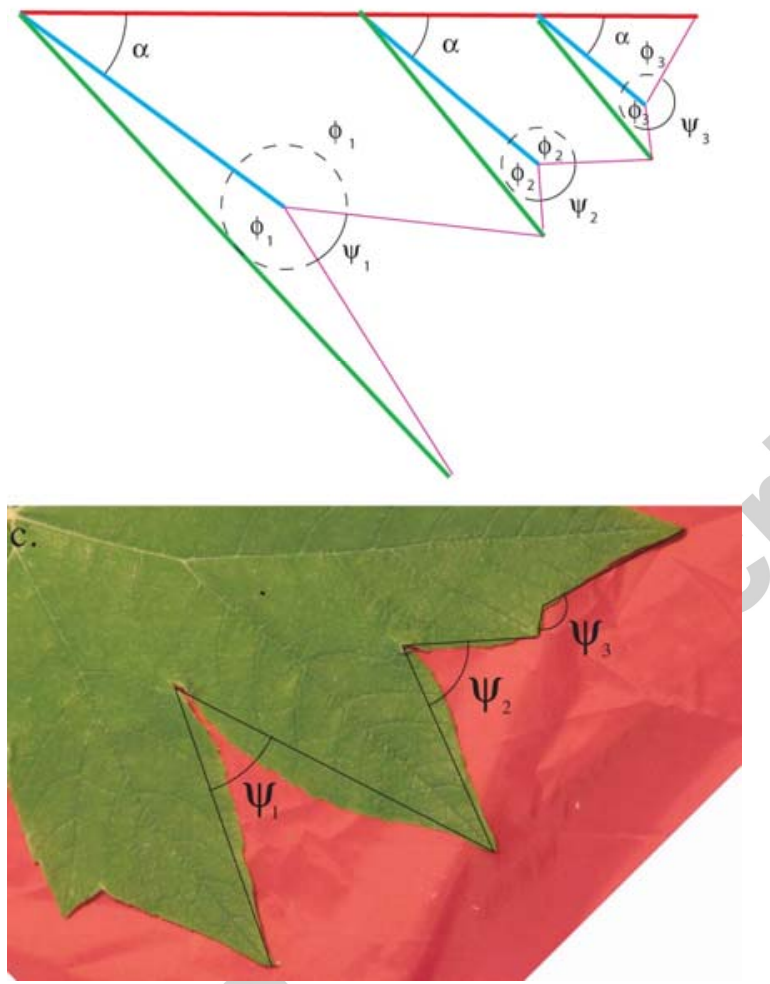

Figure 12: Consequence of secondary folds on the opening angles of the successive sinuses. a: Main vein (red) is bent by each secondary fold (green) and antifold (cyan). The angle between antifold and vein does not depend on its positions along the vein. For this reason, the angle $\phi$ between the antifold and the cut decreases along the vein. b: For this reason, the opening angle $\psi$ of the sinus increases along the lobe. c: Three successive sinuses along a lobe of Tetrapanax papyrifer. The opening angle increases toward the apical tip: $\psi_{1}<\psi_{2}<\psi_{3}$. 

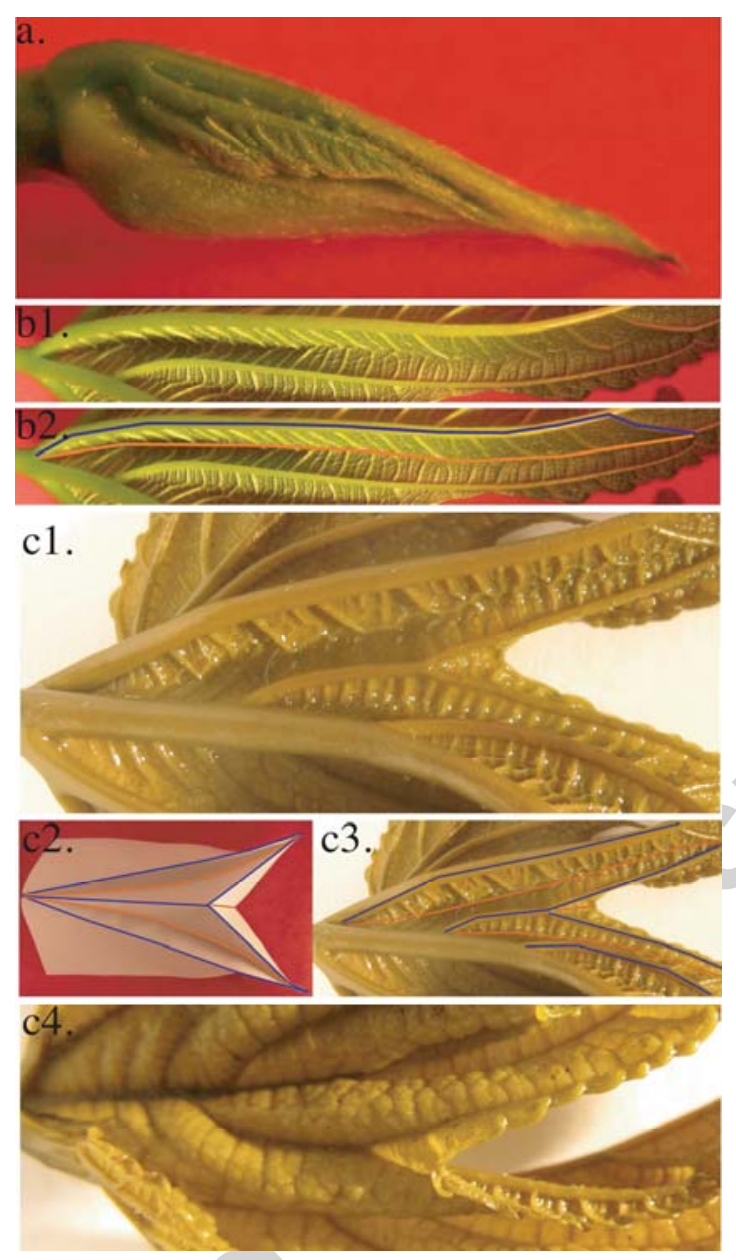

Figure 13. Particular folds of Morus Platanifolium leaves. a: A Morus platanifolium bud. The leaf is folded with its margin on the smaller bud envelope. b1: A Morus platanifolium leaf fold, which ends before the margin of the leaf without creating sinus. b2: Sketch of the folds network. (Blue lines correspond to the folds, and orange lines to the antifolds.) c1: A Morus platanifolium leaf fold (along a vein) inverts itself in an antifold just before the leaf margin (abaxial view). c2: Simplified fold network using a sheet of paper. c3: Fold network of c1. c4: Adaxial view of the same inverted fold. 


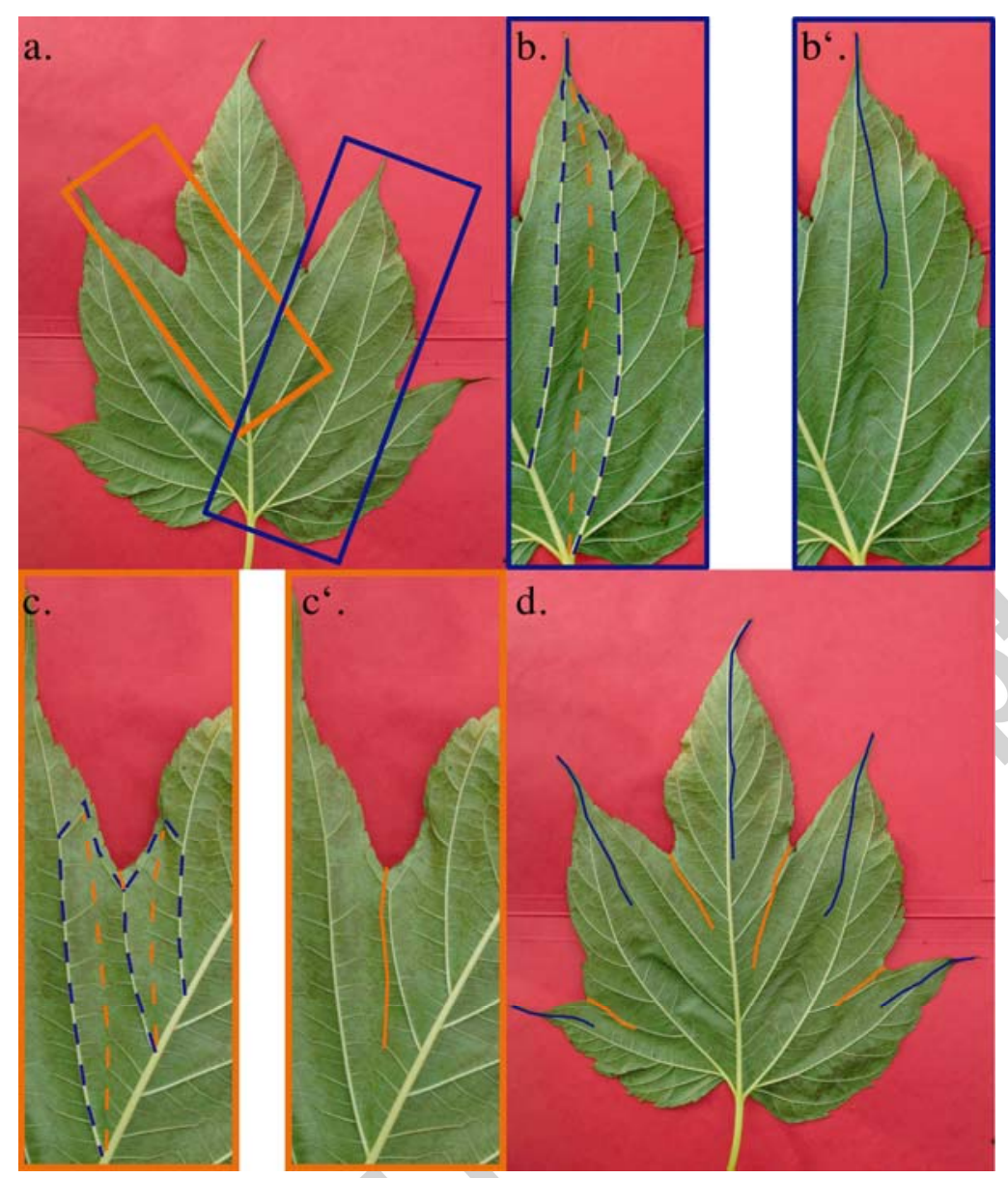

Figure. 14 : Symmetry rules are inverted in non planar leaves. a : A Morus platanifolium leaf. b: The most basal fold (dashed blue line) originates at the same point as the antifold (orange dashed line). They merge at the lobe tip without creating either another lobe or another sinus. b': The medial axis of the lobe (blue line) follows the antifold, unlike in the case of Acer pseudoplatanus, whose medial axes follow the fold (Figure 5c). c: The folds (dashed blue line) and the antifolds (orange dashed line) create a complicated network, which is an inverted fold. c': The medial axis of the sinus, when rotated $180^{\circ}$ around the sinus point (orange line) or the symmetric of the medial axis, follows mainly the vein and not the antifold, as in the case of Acer pseudoplatanus (Figure 5b). d : A Morus platanifolium leaf. Symmetry rules are inverted : The symmetry of the medial axis of the sinus (orange line) often corresponds to a vein, and the medial axis of the lobe (blue line) often corresponds to an antifold. 

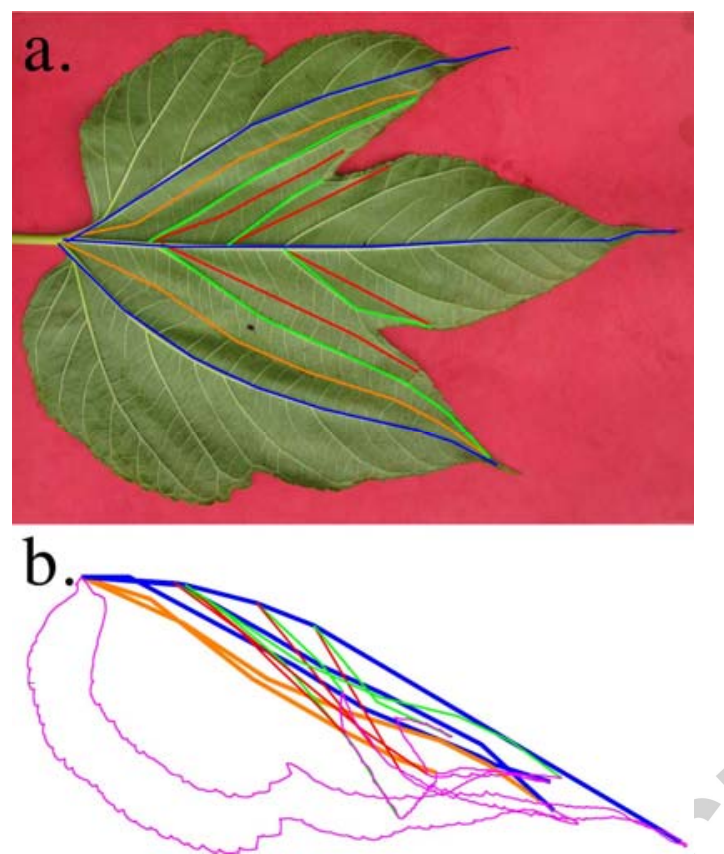

Figure 15. Numerical folding of a Morus Platanifolium leaf. a: A mature leaf of Morus platanifolium. b: Same leaf numerically folded back. The leaf contour is pink. Primary and secondary veins are colored blue and red, respectively. Primary and secondary anti-veins are marked yellow and green, respectively. Synclinal folds run along segments (anti-veins), linking a sinus to the branching point of the two surrounding veins. The thickness of the leaf is not taken into account, and the leaf is folded back onto a plane, approximating the angles as accurately as possible (see Annex).

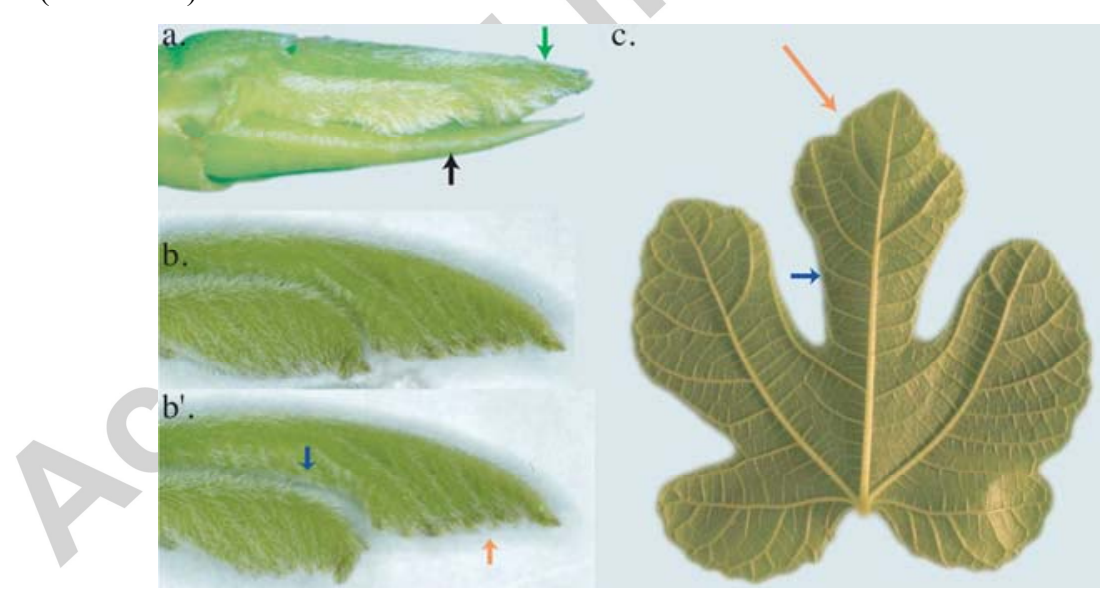

Figure 16. Particularity of Ficus Carica leaf folding. a: A Ficus Carica bud. The leaf (green arrow) is limited by another smaller bud in front (black arrow). b: A single leaf of Ficus Carica. b': The same leaf with the central lobe slightly moved. It reveals that its lower border lies on the lateral lobe (blue arrow), except at the end where it remains along the stipules (orange arrow). c: A mature Ficus Carica leaf, showing the two parts indicated in b', the part limited by the side veins (blue arrow) and the part limited by the stipules (orange arrow). 


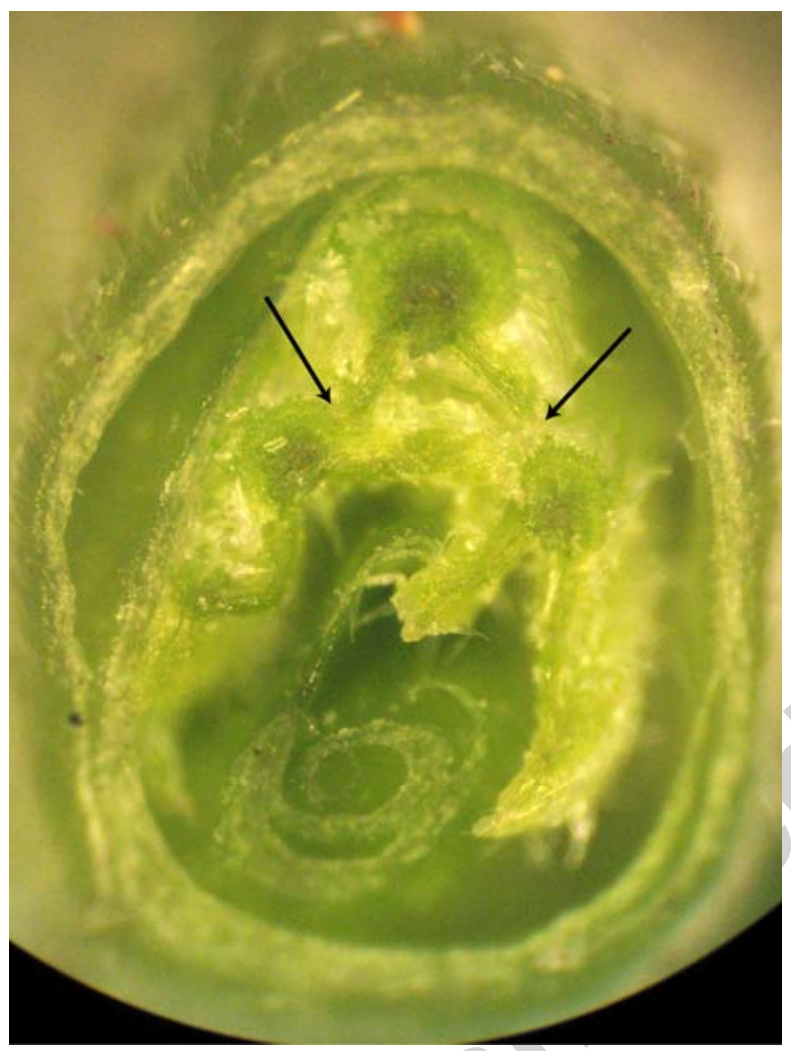

Figure 17: A transverse cut of a Ficus Carica bud. The lamina folded around the central vein ends near the lateral veins, while the lamina of the lateral lobes ends at the lower stipules.

a.

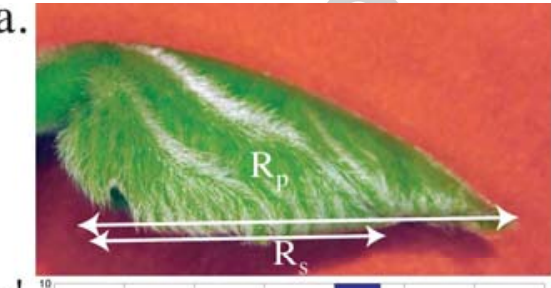

$a^{\prime}$

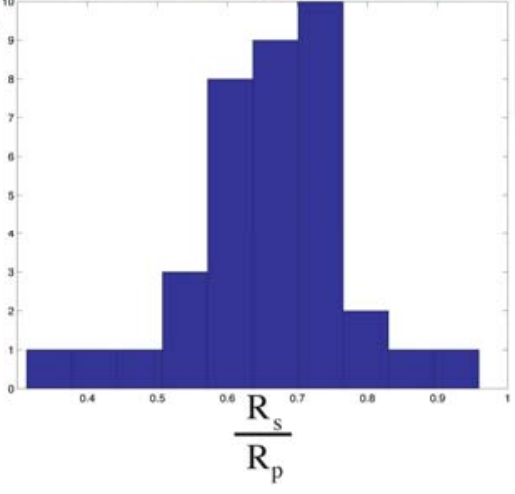

b.

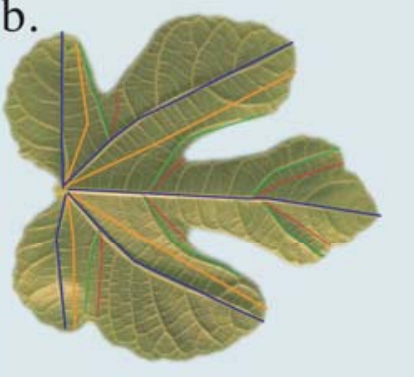

$b^{\prime}$.

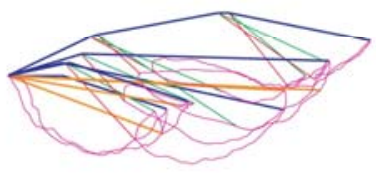

Figure 18. Aspect ratios and folding of Ficus Carica leaf. a: $R_{p}$ is the length of the entire edge of the leaf. $R_{s}$ is the length of the edge, not including the central lobe. a': Histogram of the ratio $R_{p} / R_{s}$, showing large variations. b: A mature leaf of Ficus Carica. Blue, orange, green, and red lines indicate principal folds (lobes), main 
antifolds or valley folds (sinuses), secondary folds (lobes), and secondary antifolds (sinuses), respectively. b': The same leaf folded numerically (see Annex).
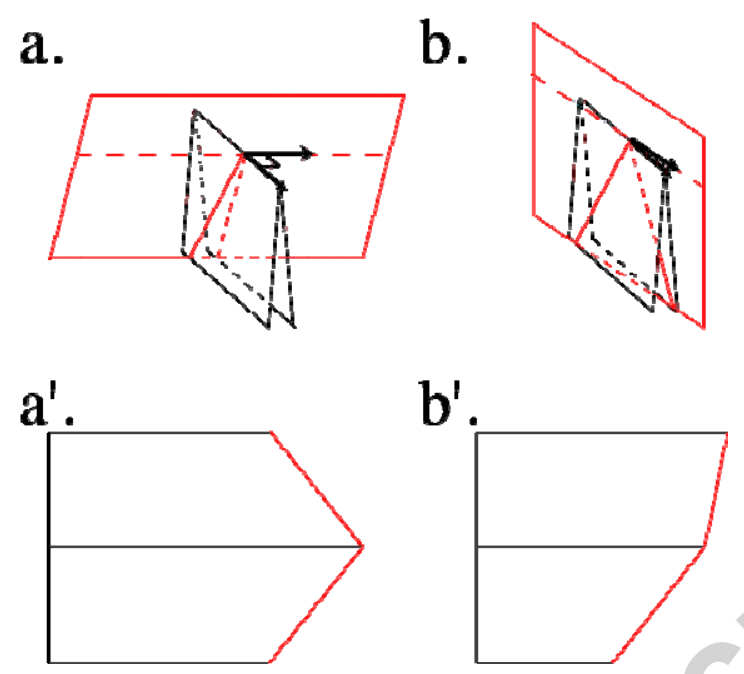

Figure 19. Different geometries of cuts. a : Transverse cut of a fold, the cut plane is orthogonal to the half-planes on the both sides of the fold. a' : Same fold as in a.,unfolded. The fold is the axis of symmetry of the edge (in red). $\mathrm{b}$ : Tangential cut of a fold. The cut plane is nearly tangential to the half-planes on both sides of the fold. b' : Same fold as in b. when unfolded. The fold is no longer the axis of symmetry of the edge (see Annex, 3D Cuts geometry).

a.

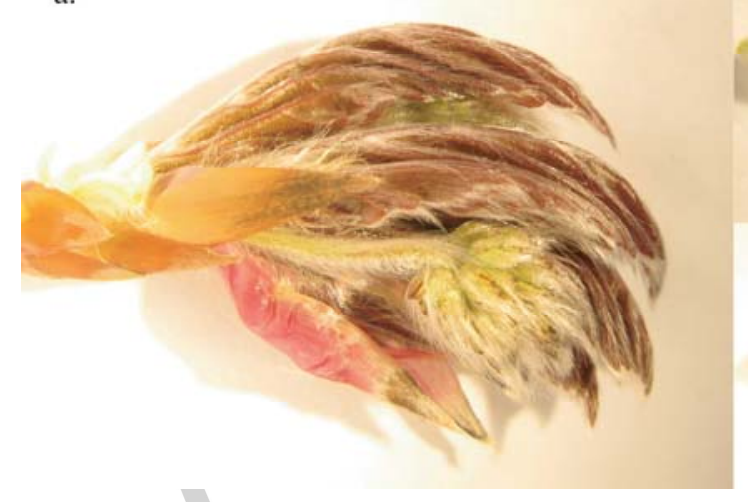

b.

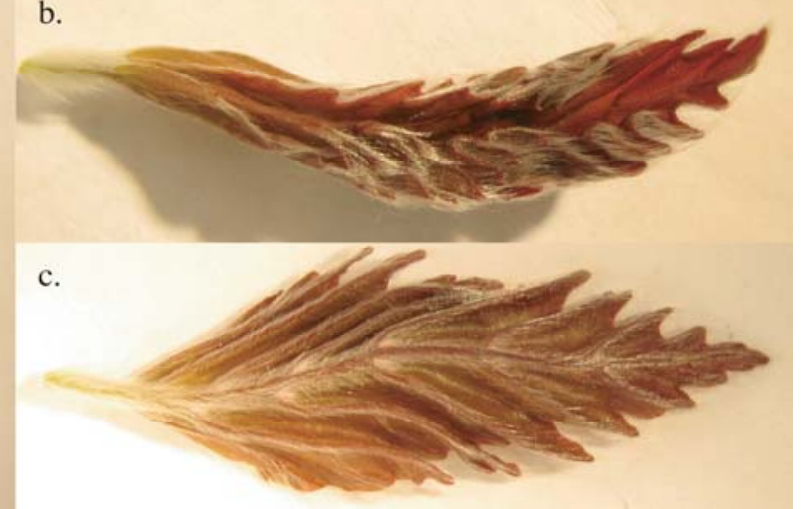

Figure 20. Fagus sylvatica (Rohan obelix var.) bud and leaf. a: A bud with folded leaves. Each leaf margin is folded on the back of the previous leaf, transversaly to the fold. b: Front (adaxial) view of a Fagus sylvatica (Rohan obelix var.) leaf and c: back (abaxial) view. Folds and antifolds are axes of symmetry of the margin. 


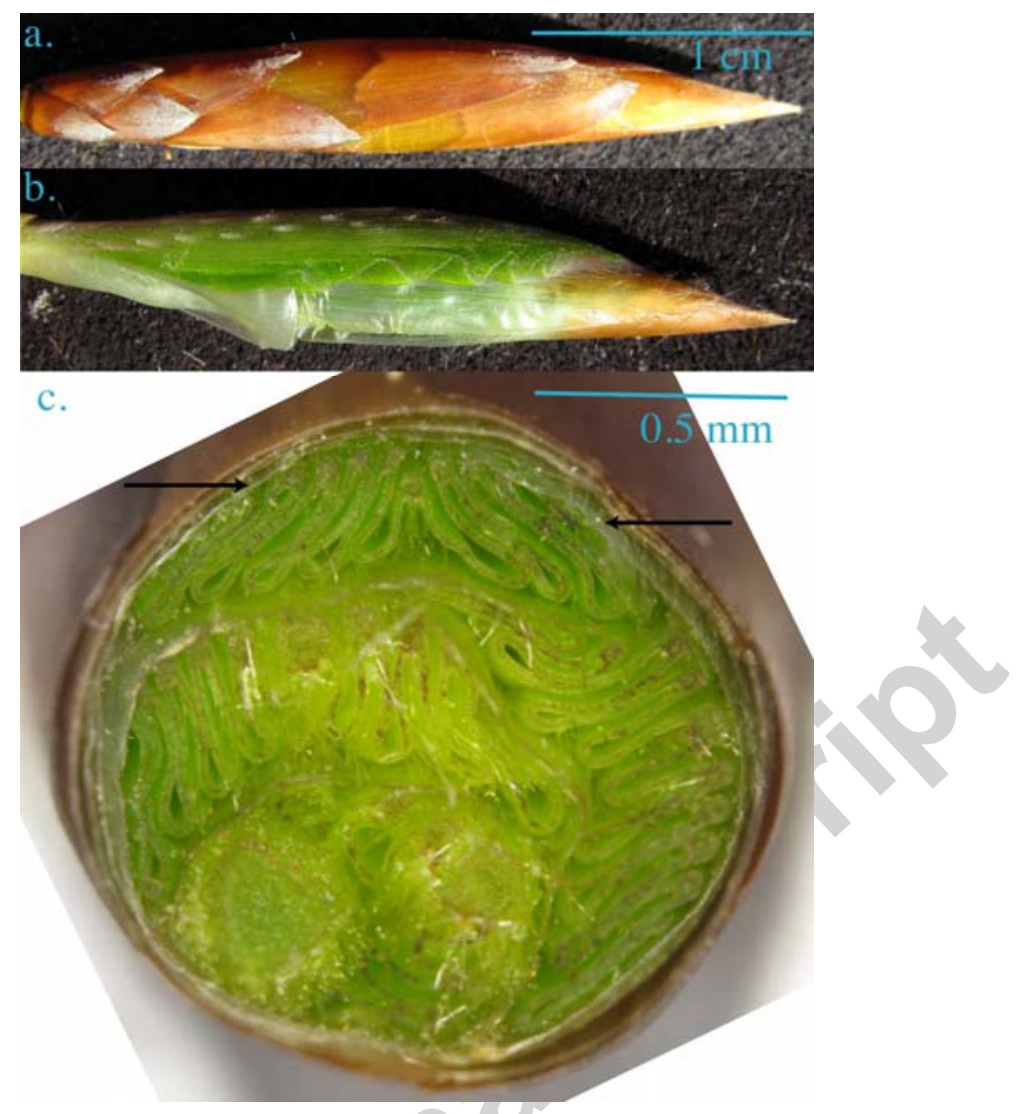

Figure 21. Organization of a wild Fagus sylvatica bud. a: A bud of Fagus sylvatica b: The same bud whithout its upper outer-shell. The fold of the leaves are not axes of symmetry. c: A transversal cut of a bud. The folded leaf margin lies on the abaxial envelope. (black arrows).

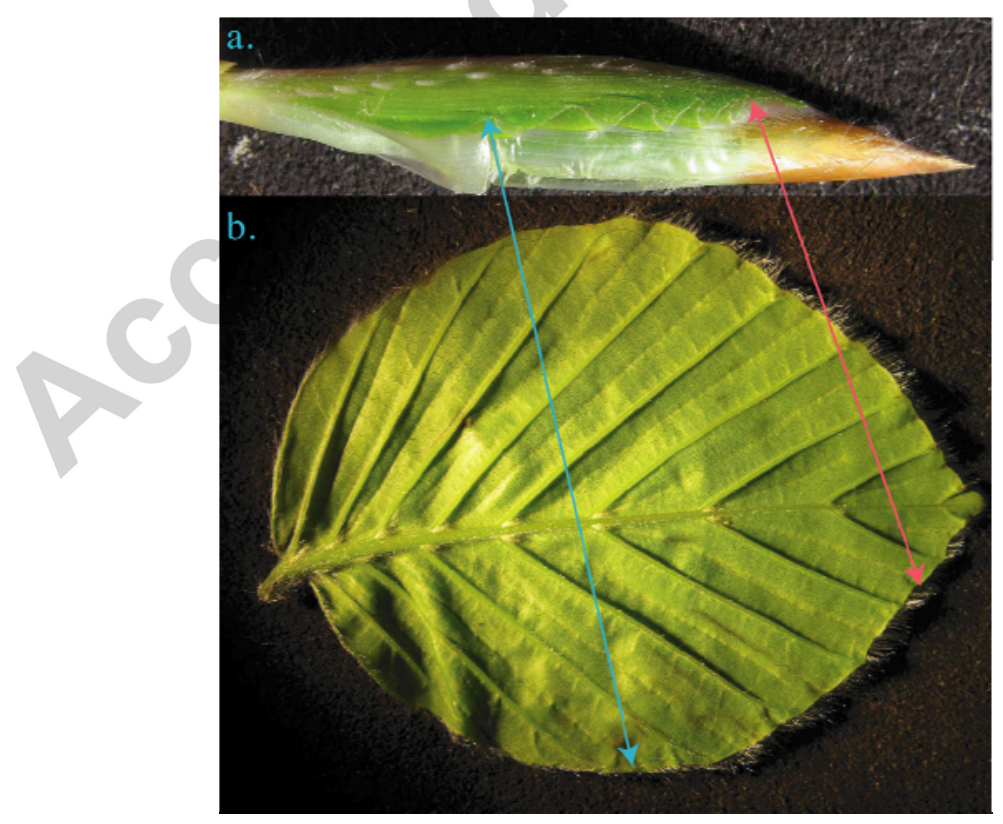

Figure 22. Relation of folding and leaf margin in wild Fagus sylvatica. a. A bud of wild Fagus sylvatica without its upper outer shell. The fold of the leaves are not axes of symmetry. b: A mature leaf. First folds along the central vein are not the axes of symmetry of the margin (blue array), because they correspond to folds tangential 
to the outer shell. Also, because the folds (which in this case are along the veins) do not lie on top of each other due to the thickness of the veins, the antifold is not equi-distant from the two nearby folds. The last secondary folds along the central vein are the axes of symmetry of the margin, because they correspond to the folds, which are transverse to the outer shell (red array).
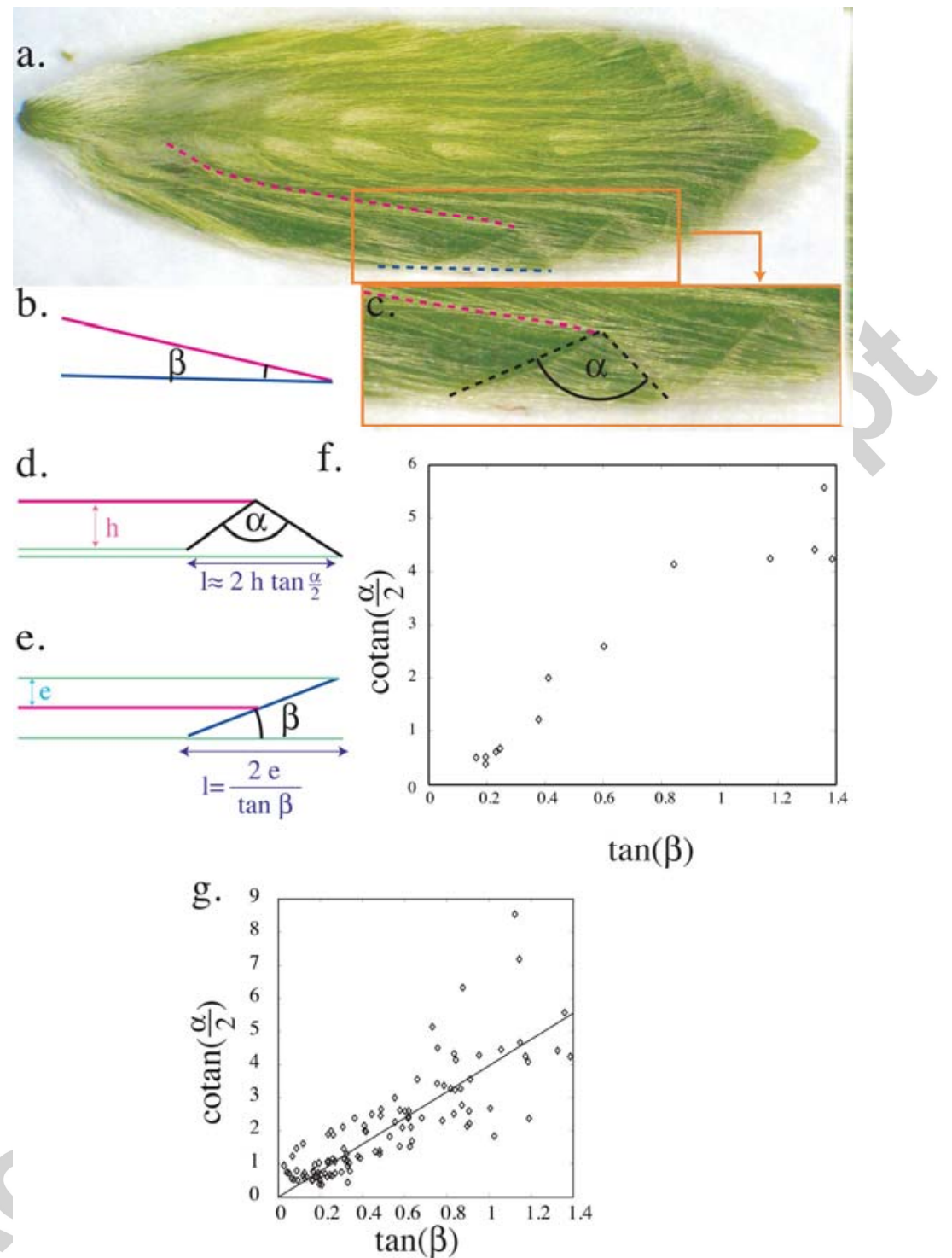

Figure 23. Geometrical relationships of tangential cutting of beech leaves. a: Schema of measurements on the abaxial view of a beech leaf. Magenta dashed line corresponds to the fold, which lies along a vein. Blue dashed line links two consecutive sinuses centered around this vein fold. $b$ : $\beta$ is the angle between the fit of the blue dashed line (represented by a blue continuous line) and the fit of the magenta dashed line (represented by a magenta continuous line). c: The angle $\alpha$ (black) corresponds to the opening of the cut, and to its asymetry with the folds. $d$ : Schema of a lateral view of a fold. First way to calculate the length 1 with the angle of asymetry $\alpha$. e: Schema of an upper view of an unfolded fold. Second way to calculate the length 1 with the border angle $\beta$. f: $\operatorname{cotan}(\alpha / 2)$ as a function of $\tan (\beta)$ for the leaf a. $g \cdot \operatorname{cotan}(\alpha / 2)$ as a function of $\tan (\beta)$ for 8 different leaves. 


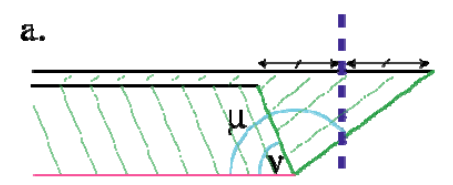

b.

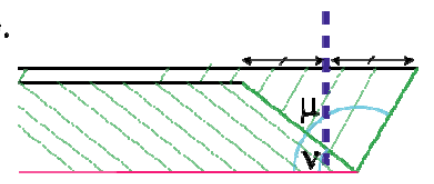

$a^{*}$.

$\mathbf{b}^{4}$
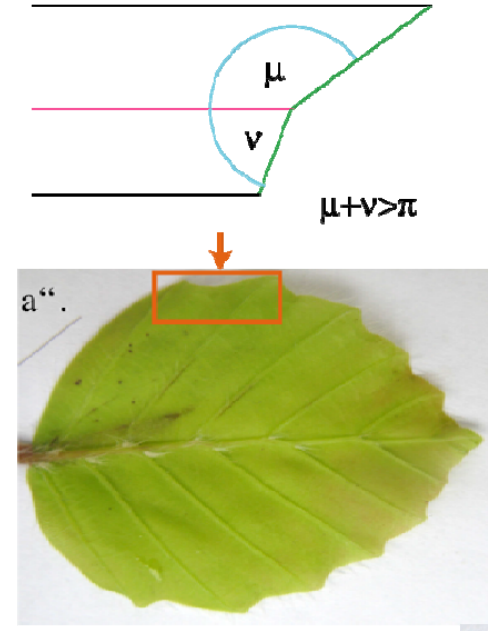

$\mu+v<\pi$

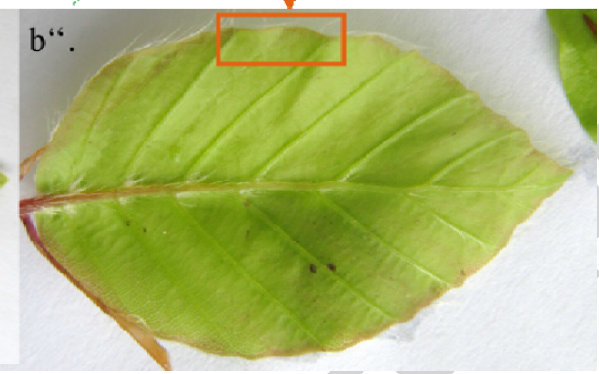

Figure 24. Possible geometries of transverse cuts in beech leaves. The black, red, and green lines correspond to veins, an antivein fold, and the leaf margin, respectively. a: If the antifold's end is closer to the tip of the first vein than to the tip of the second vein, then $a^{\prime}: \mu+v>\pi$, and it will yield a sinus when unfolded. a": Beech leaf whose antifolds become sinuses (orange arrow). b-b'-b": If the antifold's end is closer to the tip of the second vein than to the tip of the first vein, then $\mu+v<\pi$. It will yield a beech leaf whose antivein folds become lobes (orange arrows) (see Annex, 3D Cuts geometry).

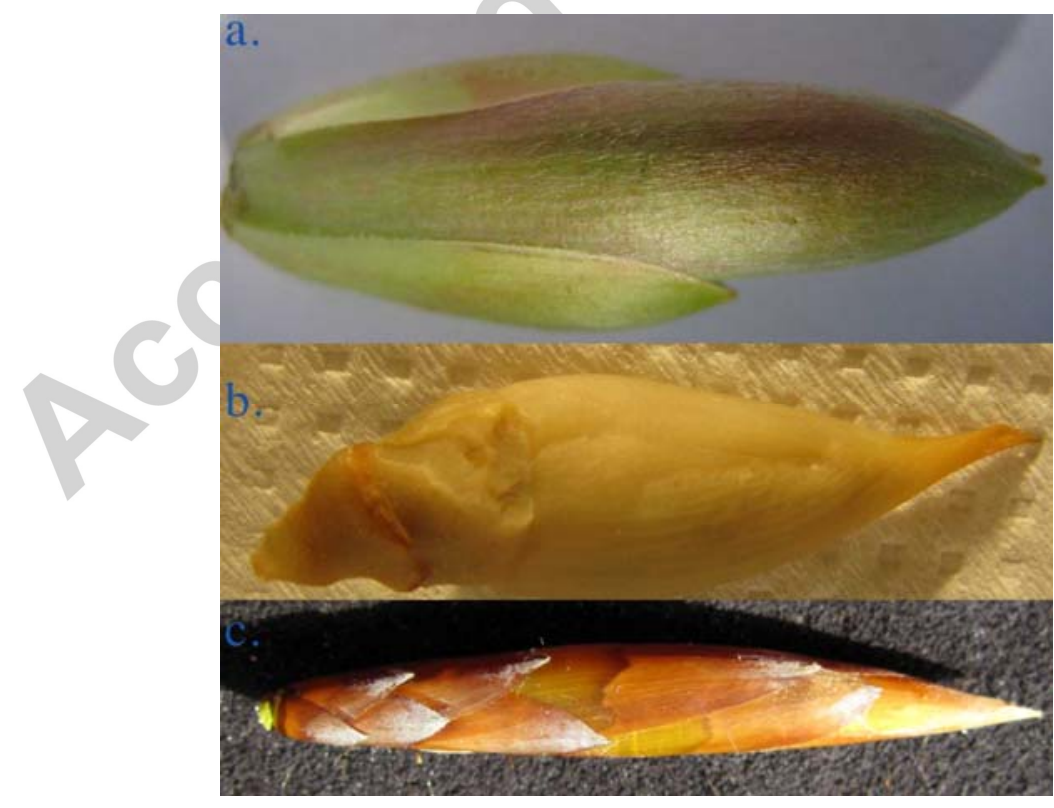

Figure 25. Different bud shapes. a: Acer pseudoplatanus. b: Ficus Carica. c: Fagus sylvatica. The shapes of the bud $\mathrm{b}$ and $\mathrm{c}$ are more acute than the first one. 

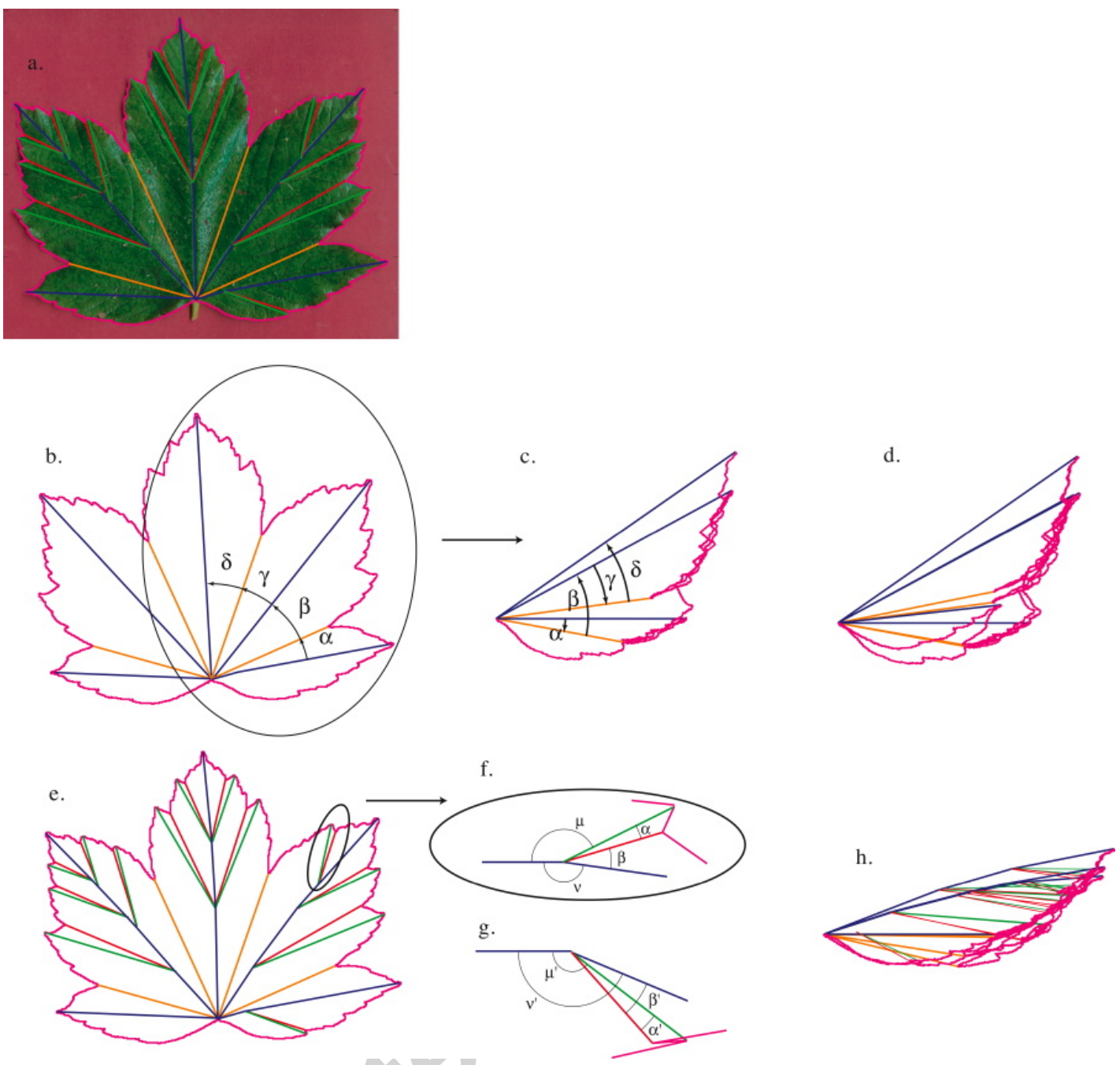

Figure 26 a. On a maple leaf, the first order veins (blue lines) and secondary veins (green lines) are drawn. The first order anti-veins (orange lines), which extend from the intersection of two veins up to the sinus between these veins, and the second order anti-veins (red lines) are also drawn. The contour of the leaf (mangenta line) is numerically detected. b. The result with the contour and only the main veins and anti-veins, and the first angles between them. c. Half part of the precedent sketch once refolded. $\alpha$ and $\gamma$ were reversed, and their respective contour were drawn inversed. d. The refolded leaf using only its main folds. e. Sketch of the leaf with all its folds: secondary one and main one. f. Scheme of a secondary fold, unfolded. g. Folded in a plane. The new angles are obtained as described in the text. h. The whole set of veins and anti-veins is then drawn, with their respective contour, giving the completely refolded leaf. 


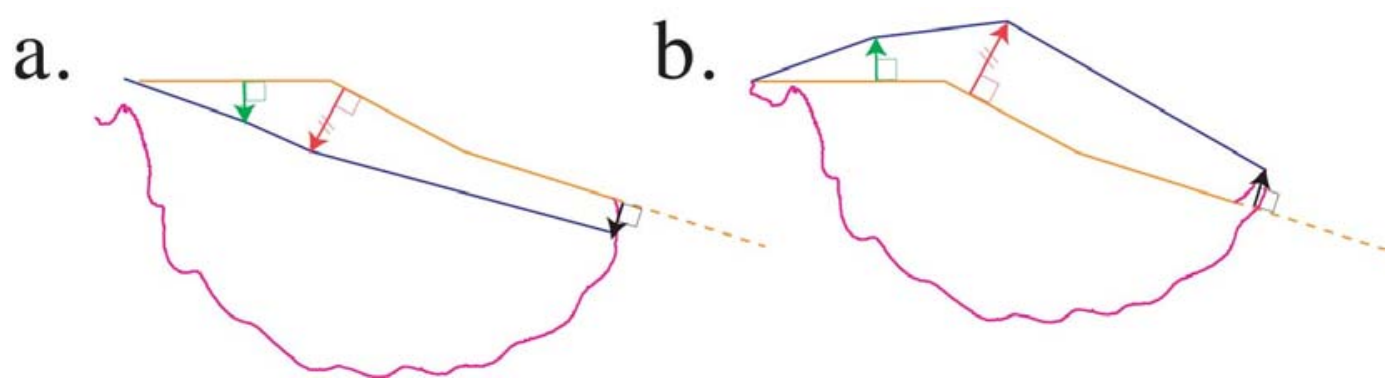

Figure 27: Symmetry around a curved fold. a. An antifold was drawn with an orange line, a fold by a blue one, and the leaf margin by a magenta one. b. After the symmetry of the fold using the antifold as axis of symmetry. 


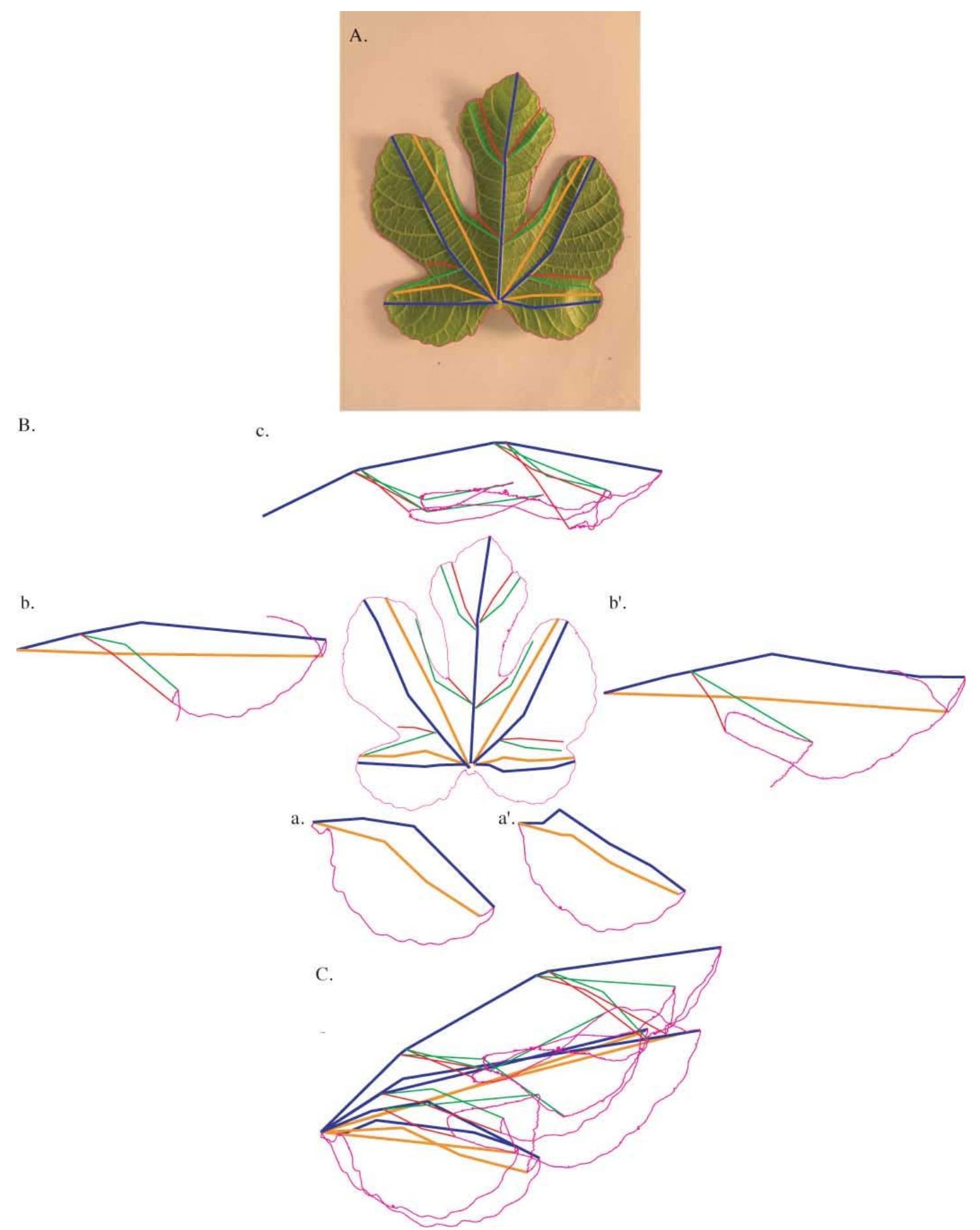

Figure 28: A. Coordinate of folds (along the veins) and antiveins (the zone where secondary veins join) are measured on a Ficus Carica leaf image. B. Folding method. a. We orientate the left lateral lobe symetric (using the antifold as axias) in such way that the first segment of the fold is horizontal. b. We draw the following fold symetric turned with the angle between this fold and the precedent antifold. c. We represent the central lobe with its secondary folds. a'. b'. Idem of $a$. and b on the right side of the leaf. C. Final result. 

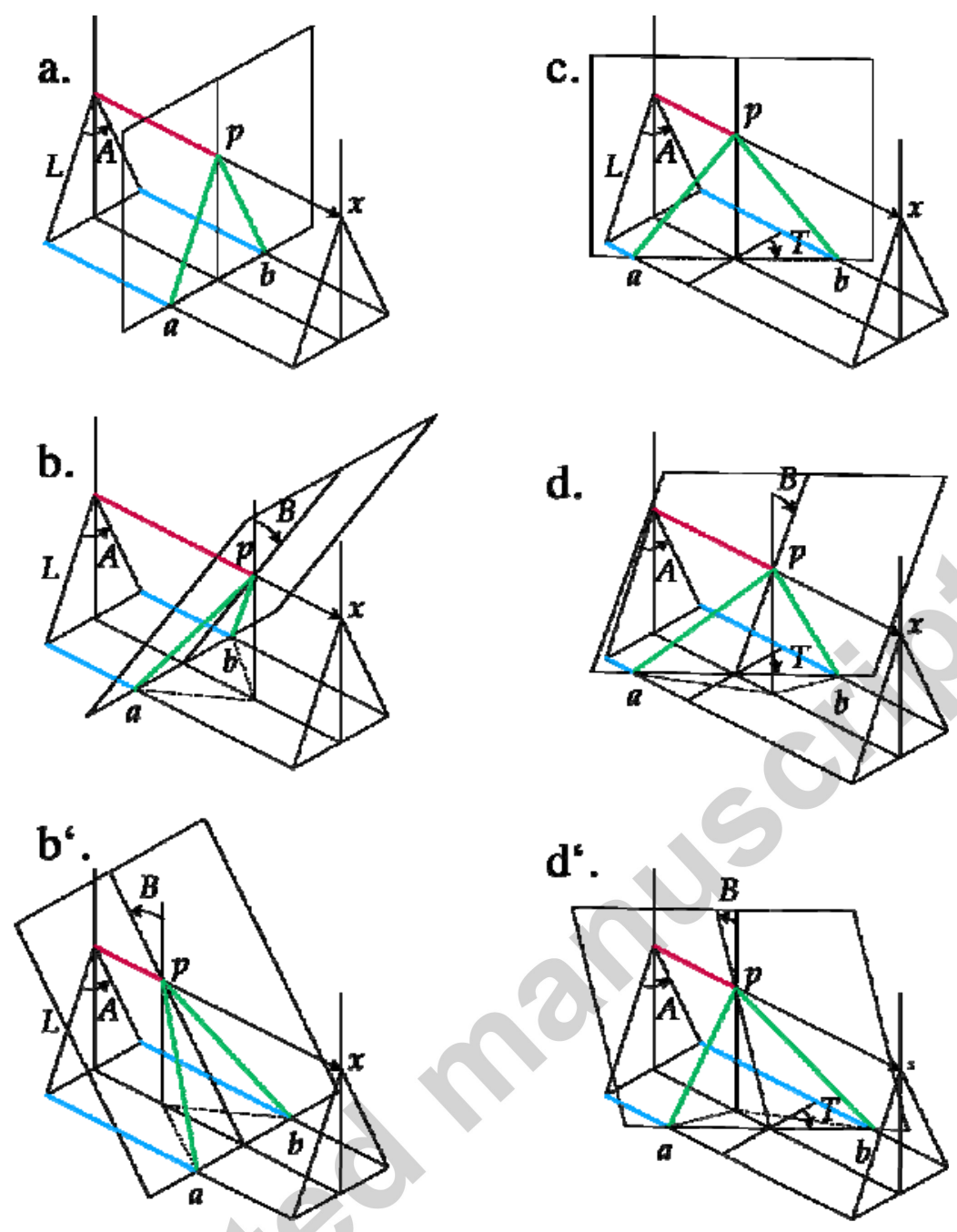

Figure 29 : Geometry of a cut. With a lamina open with and angle A, a vein fold on top (red), and a lamina length on each side L. a : a simple perpendicular cut, leaving a flat perimeter. $\mathrm{b}:$ if the cut goes adaxially (inward), with a negative angle B, the positions on the side retract, and the vein fold correspond to the tip of a lobe, as common. b' : if the cut goes abaxially (forward), with a postive B, the side points moves foward on the contrary, leading to the rare case of a vein at a sinus. $d$ : if the cut is made tranversaly, with an angle $T$, the points remains aligned on a straight boundary but one moves foward while the other backward. d-d' : when the cut is slented in both ways, then asymatrical cuts corresponding to a point (d) or a sinus (d') can be obtained (as in : Fagus sylvatica Figure 24). 


\section{Highlights}

Leaf shape seems to be the consequence of filling the bud with a certain folding.

The fig tree and beech leaves solve two non-zero thickness "fold-and-cut" problems.

Reiteration and confinement are two important ingredients of leaf development. 